\title{
NCoR1 and SMRT fine-tune inflammatory versus tolerogenic balance in dendritic cells by differentially regulating STAT3 signaling
}

\author{
A. Jha ${ }^{1,2, \dagger}$ A. Ahad ${ }^{1,2, \dagger}$, G. P. Mishra ${ }^{1,3, \dagger}$, K. Sen ${ }^{4}$, S. Smita ${ }^{1,2}$, A. P. Minz ${ }^{1}$, \\ V. K. Biswas ${ }^{1,3}$, A.Tripathy ${ }^{3}$, S. B. Senapati ${ }^{1,2}$, B. Gupta ${ }^{3}$, H. A. Orbea ${ }^{5}$, S. K. Raghav ${ }^{1,2, *}$ \\ ${ }^{1}$ Immuno-genomics \& Systems Biology laboratory, Institute of Life Sciences (ILS), \\ Bhubaneswar, Odisha, India-751023 \\ ${ }^{2}$ Manipal Academy of Higher Education, Manipal, Karnataka-576104, India \\ ${ }^{3}$ Department of Biotechnology, Kalinga Institute of Industrial Technology (KIIT), \\ Bhubaneswar, India \\ ${ }^{4}$ Regional Centre for Biotechnology, NCR Biotech Science Cluster, Haryana, India \\ ${ }^{5}$ Department of Biochemistry CIIL, University of Lausanne (UNIL), CH-1066 \\ Epalinges, Switzerland
}

${ }^{\dagger}$ Equal contribution first author

*Corresponding author: Sunil K. Raghav, PhD.

Email for correspondence: sunilraghav@ils.res.in, raghuvanshi2010@yahoo.co.in 
Dendritic cell (DC) fine-tunes inflammatory versus tolerogenic responses to protect from immune-pathology. However, the role of co-regulators in maintaining this balance is unexplored. NCoR1-mediated repression of DC immune-tolerance has been recently reported. Here we found that depletion of NCoR1 paralog SMRT enhanced cDC1 activation and expression of IL-6, IL-12 and IL-23 while concomitantly decreasing IL-10 expression/secretion. Consequently, co-cultured $\mathrm{CD}^{+}$and $\mathrm{CD}^{+}$T-cells depicted enhanced Th1/Th17 frequency and cytotoxicity, respectively. Comparative genomic analysis demonstrated differential regulation of IL-10 by SMRT and NCoR1. SMRT depletion repressed mTOR-STAT3-IL10 signaling in cDC1 by down-regulating NR4A1. Besides, NFkBIA and SOCS3 were down-regulated in SMRT knockdown cDC1, supporting increased production of inflammatory cytokines. Moreover, adoptive transfer of SMRT knockdown cDC1 in OVA-DTH induced footpad inflammation led to increased Th1/Th17 and reduced tumor burden after B16 melanoma injection by enhancing oncolytic $\mathrm{CD}^{+}$T-cell frequency, respectively. We also depicted decreased Smrt expression in Rheumatoid Arthritis, a Th1/Th17 disease. 


\section{Introduction}

Dendritic cells (DCs) play an important role in immune surveillance and maintains an optimal balance between inflammation and immune-tolerance to avoid immune-pathology $[1,2]$. After encountering pathogens DCs undergo activation and maturation leading to secretion of cytokines and expression of co-stimulatory molecules, which ultimately decides the fate of a particular T-cell response to clear the invading pathogens [3]. Hence, antigen specific activation of DCs is an important event to orchestrate the immune system culminating into development of T-cell adaptive response for the induction and expansion of either an optimum protective pro-inflammatory or tolerogenic response [4]. DC subtype specific differential expression of toll like receptors (TLRs) along with their unique signaling cascades provide the specificity against the pathogens e.g., plasmacytoid DCs (pDCs) highly express TLR7 and TLR9, required to mount antiviral response whereas conventional DCs (cDCs) are mainly responsible to maintain the balance between inflammatory vs tolerogenic response and cross presentation [5-7]. Among cDCs, cDC1s $\left(\mathrm{CD} 8 \alpha^{+}\right.$DCs) are considered as bystanders which integrate signals derived from intracellular infection to tailor the appropriate CD4 ${ }^{+} \mathrm{T}$-cell response along with anti-tumor $\mathrm{CD}^{+} \mathrm{T}$-cell activation with the help of their unique property of cross presentation [8]. cDC1s identify pathogen associated molecular pattern (PAMPS), such as bacterial unmethylated CpG-DNA or viral double stranded RNA through TLR9 and TLR3 respectively [9]. Upon TLR9 stimulation in cDC1s, the TIR domain of TLR and an adapter, Myd88, activates interleukin-1 receptor-associated kinase-4 (IRAK4) and IRAK1 [10]. IRAK4 subsequently activates the NF-kB signaling [11]. The TLR9-Myd88 signaling has also been linked with JAK-STAT signaling pathway for cytokines production [12]. STAT3 is a new entry in the block although the TLR9-Myd88-STAT3 signaling does not affect NF-kB signaling [9]. STAT3 depleted DCs have been shown to be insensitive to IL-10 mediated suppression leading to hyper-activation of T-cells and inflammation in mice [13]. A combination of such divergent DC signals leads to differentiation of naive T helper cells to various effector subtypes such as Th1, Th2, Th17 or Tregs. A fine balance of secretory cytokines like IL-6, IL-12, and IL-23 modulates Th cells towards Th1 or Th17 subtypes, whereas increased levels of IL-10, SOCS3 and CD83 differentiate them towards Tregs [14]. Thus, a tight regulation of these cytokines is important to maintain the fine balance between inflammatory, anti-inflammatory or tolerogenic responses [15]. The idea of perturbing Tcell differentiation to modulate the immune system for cell mediated therapy is an accepted concept. For example, the use of DCs to manipulate T-cells in cancer immunotherapy has 
been widely reported. Despite a number of attempts, there are multiple occurrences of insufficient T-cell activation and effective priming in in vivo systems [8]. Therefore, identifying ways to perturb DC responses in a controlled manner to enhance T-cell function is an interesting area. Recently, NF-kB signaling was shown to be perturbed in tolerogenic DCs having increased IL-10 production [16]. However, the regulators underlying the fine modulation of these TFs are not clearly documented. A group of TFs belong to nuclear receptors (NRs) such as NURR-77 or NR4A1 and PPAR- $\gamma$ are reported to have a role in regulation of cytokine gene expression [17]. NURR-77 controls production of IL-6, TNF$\alpha$, and IL-12 in both human and murine dendritic cells [18]. Similarly, peroxisome proliferator-activated receptor (PPAR- $\gamma$ ) also exerts anti-inflammatory effects in monocytes and macrophages [18]. Although TFs and NRs lead to activation of transcription, their activity is tightly regulated by a network of co-regulators, including co-activators and corepressors. Nuclear receptor co-repressor 1 (NCoR1) and its paralog Silencing mediator of retinoic acid and thyroid hormone receptor (SMRT) were identified in relation to unliganded thyroid and retinoic acid receptor mediated repression of gene expression [19]. Ghisletti et. al. has shown that the combination of NCoR1 and SMRT is required for regulation of inflammatory and anti-inflammatory genes in macrophages [20]. However, the combinatorial role of NCoR1 and SMRT in immune response regulation in DCs is largely unexplored. Recently we demonstrated that active repression of tolerogenic genes like IL10 by NCoR1 is essential for development of immunogenic response in DCs [21]. NCoR1 depletion enhanced the expression of tolerogenic molecules like IL-10, IL-27, PDL1 and SOCS3 resulting in increased frequency of Tregs and shift of immunogenic balance towards tolerance [21].

In this study, we explored the role of SMRT in modulating the immune function of cDC1 DCs in vitro, ex vivo and in vivo. We identified how two highly homologous nuclear receptor co-repressor proteins NCoR1 and SMRT tightly control the fine balance of inflammatory and tolerogenic response in DCs, which consequently regulates the differentiation of naïve Th cells into Th1, Th17 or Tregs. Moreover, comparative genomic analysis of NCoR1 and SMRT depleted cDC1 revealed their differential role in control of STAT3 signaling and IL10 expression and its underlying control of repression. Overall, our study demonstrated that NCoR1 and SMRT are potential targets for regulating a fine balance of DC mediated inflammatory and tolerogenic T-cell responses. 


\section{Results}

\section{SMRT KD cDC1 DCs depicted enhanced activation / co-stimulation}

We observed that Nuclear Receptor Co-repressor-2 (Ncor2 or Smrt) was constitutively expressed at transcript levels in murine cDC1 (mutu-cDC1 line) before and after 2h, $6 \mathrm{~h}$ and 12h of activation by TLR9 ligand, CpG-B [22] (Fig. S1A). To identify the potential role of SMRT in cDC1, we generated stable SMRT gene knock down (SMRT KD) and empty vector transduced (control) cDC1 mutu-cDC1 lines. SMRT gene depletion was confirmed at transcript (>75-80\%) and protein level (>50-80\%) in both, unstimulated and 6h CpG challenged conditions (Fig. 1A and 1B). Then we performed RT-qPCR for proinflammatory and anti-inflammatory cytokine gene transcripts (Il-12b \& Il-10) in control and SMRT KD cDC1 cells before and after 2h and 6h CpG challenge. We found significantly increased $I l-12 b$ with a concomitantly decreased $I l-10$ post CpG stimulation in SMRT KD cDC1 compared to control cells (Fig. 1A). Besides, flow cytometric analysis showed significantly increased percentage positive cells for CD80, CD86 and CD40 in SMRT KD cDC1 as compared to controls. CD80 was increased only in activated conditions (Fig. 1C). The median fluorescence intensity (MFI) shifts also showed a similar trend (Fig. S1B). We observed that SMRT KD cDC1 preserved the similar phenotype under TLR3 stimulation with pIC as well (Fig. S1C). Next, we investigated the antigen presentation ability by assessing expression of MHC-I and MHC-II on SMRT depleted DCs. We found significantly increased MHC-I percent positive cells in both CpG and pIC activation whereas MHC-II levels remained unchanged (Fig. 1C and Fig. S1C). Gating strategy used for mutuDC cell line is uniform for all experiments (Fig. S1D). MFI shifts depicted similar trends. To further validate the impact of SMRT depletion in primary ex vivo cDC1s, we performed transient SMRT depletion in primary bone-marrow derived cDC1s (BMcDC1s) cultured in FLT3L. We found that CD80 and CD86 showed an increased trend in SMRT KD condition as compared to controls (Fig. 1D and Fig. S2A). However, we did not observe any change in MHC-II similar to in vitro results upon SMRT depletion in primary cDC1s (Fig. S2A). Uniform gating strategies were used throughout BMcDC1analysis (Fig. S2B). 


\section{SMRT depleted cDC1 showed increased IL-6, IL-12 and IL-23 pro-inflammatory cytokines with concomitantly decreased IL-10}

Next, we assessed the expression of important DC response cytokines in both, mutu-cDC1 line and primary BMcDC1s. We found that SMRT depletion significantly enhanced the percent positive cells as well as MFI shifts for IL-6, IL-12p40 and IL-23p19 cytokines after 6h CpG challenge as compared to control cDC1. IL-23p19 was significantly increased in SMRT KD cDC1 irrespective of stimulation (Fig. 2A and Fig. S3A). On the contrary, we found that IL-10 was significantly reduced upon activation in SMRT KD DCs (Fig. 2A and Fig. S3A). Similar results were observed after TLR3 stimulation with pIC (Fig. S3B). Uniform gating strategies were used throughout BMcDC1analysis (Fig. S3C). Further to estimate the levels of these cytokines in CpG activated cDC1 culture supernatants, we performed bio-plex assays and found the cytokine levels of IL-6, IL-12p40 and IL-12p70 were significantly increased in SMRT depleted mutu-cDC1 line with a drastic reduction in IL-10 (Fig. 2B). Moreover, the IL-2 cytokine necessary for clonal expansion of T-cells was found to be significantly increased in SMRT KD cDC1 compared to controls (Fig. 2B). We further confirmed these findings in primary BMcDC1s and similar to our mutuDC line, we found significantly increased IL-6 and IL-23 and decreased IL-10 cytokine in BMcDC1s as well (Fig. 2C and Fig. S4A). However, IL-12p40 percent positive cells showed significant decrease after SMRT KD in BMcDC1s (Fig. S4A, S4B). Although IL-12p40 percent positive cells in BMcDC1s was reduced in KD compared to control however, similar to mutuDC line data in bio-plex cytokine assays in control and SMRT KD BMcDC1s showed a significant increase in secreted IL-12p40 after 6h CpG-B stimulation (Fig. 2D). IL-6, IL12p70, and IL-2 also showed a non-significant but increasing trend (Fig. 2D). These observations suggested towards a strong inflammatory phenotype for SMRT KD cDC1.

\section{Co-culture of OT-II CD4 ${ }^{+}$Th cells with SMRT depleted cDC1 enhanced Th1 and Th17 differentiation}

To understand the functional impact of perturbed SMRT depleted DC responses on CD4 ${ }^{+}$ Th cell differentiation, we performed a co-culture experiment of $\mathrm{CD}^{+}$Th cells isolated from OT-II transgenic mice with SMRT KD and control cDC1 mutuDC line. DCs were pulsed with OT-II peptide overnight, followed by activating with CpG or pIC for $2 \mathrm{~h}$ before addition of OT-II T-cells. The isolated $\mathrm{CD}^{+}$OT-II were labelled with efluor-670 proliferation dye and co-cultured with OVA pulsed and CpG or pIC activated DCs for 72h. 
We first looked into the proliferation of co-cultured T-cells and found that in SMRT KD conditions there was a significantly enhanced T-cell proliferation compared to controls (Fig. 3A-3C and Fig. S5A). Then we profiled the T-cell subtype patterns to look for its impact on differentiation. It is known that IL-6 leads to suppression of fork head box protein P3 (FOXP3) TF expression thus repressing Treg differentiation [23]. IL-6 along with IL-23 contributes to the development of Th17 cells by inducing ROR $\gamma \mathrm{t}$ expression [24]. Also, IL12 p70 is known to upregulate T-bet generating Th1 subtype. In our experiment, we found a

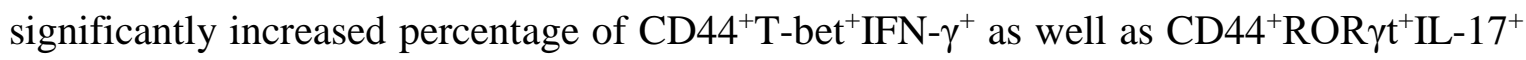
cells supporting enhanced frequency of Th1 and Th17 subtype in activated SMRT KD cDC1 condition as compared to control DCs (Fig. 3D-3E). A similar increase in CD44 $4^{+}$T-bet ${ }^{+}$IFN$\gamma^{+}$cells was observed in pIC stimulated condition as well (Fig. S5B). To further confirm the increased Th17 polarization, we checked IL-17 cytokine levels in the culture supernatants and found it to be significantly increased (Fig. 3F). No significant change was observed in IL-13 secretion (Fig. S5C). There was no significant change observed for IL-10, Foxp3 ${ }^{+}$ Tregs or GATA $3^{+}$Th2 cells (Fig. S5D). The gating strategy used for the analysis is depicted (Fig. S5E).

\section{Smrt expression is decreased in PBMCs of Rheumatoid Arthritis (RA) patients}

The autoimmune diseases like Rheumatoid Arthritis (RA) have been widely classified as Th1 and Th17 disease and IL-10 expression is also found to be drastically reduced [25]. It had been established earlier that Th1 responses were involved in autoimmune disease. However, work on IFN- $\gamma$ and IL-12 $2^{-/}$mice showed that these mice have a high probability of developing collagen-induced arthritis. With this came the advent of Th17 cells and their role in autoimmunity [26]. Therefore, to identify if in such diseases Smrt expression is decreased and if it correlates with inflammatory phenotype, we performed RT-qPCR of Smrt from peripheral blood mononuclear cells (PBMCs) of 14 healthy donors and 11 RA patients. Our results demonstrated significantly lower Smrt expression in RA patients compared to their healthy counterparts (Fig. 3G). RA patients with disease activity score (DAS) above 4 has been considered in the study. This result suggested that Smrt decrease could be associated with increased inflammatory phenotype in RA disease. 


\section{Co-culture of SMRT KD cDC1 with OT-I CD8 ${ }^{+}$T-cells increases T-cell cytotoxicity}

As we observed significantly increased MHC-I expression on SMRT depleted DCs, we were interested to identify if these DCs have the potential to increase cytotoxic activity of $\mathrm{CD}^{+} \mathrm{T}$-cells. For the same, $\mathrm{CD} 8^{+} \mathrm{T}$-cells isolated from the spleen of OT-I transgenic mice were co-cultured with control and SMRT KD cDC1s. DCs were first pulsed with SIINFEKL (OVA peptide 257-264) with or without CpG or pIC for $2 \mathrm{~h}$ followed by co-culture with purified efluor-670 labelled $\mathrm{CD} 8^{+}$T-cells for $72 \mathrm{~h}$ to assess proliferation and its impact on IFN- $\gamma$, perforin and granzyme-B (GrB) expression. As similar to CD4 ${ }^{+}$T-cells, we observed a significantly increased proliferation of co-cultured CD8 ${ }^{+}$T-cells in SMRT KD condition compared to controls (Fig. 4A and 4B). At the same time, we found an increased frequency of $\mathrm{CD}^{+} \mathrm{CD}_{4} 4^{+} \mathrm{T}$-cells expressing IFN- $\gamma, \mathrm{GrB}$, and Perforin in $\mathrm{CpG}$ activated SMRT depletion condition as compared to control (Fig. 4C). Similar results were obtained for GrB and Perforin using pIC stimulated conditions. IFN- $\gamma$ showed a non-significant but increasing trend (Fig. S5F). The gating strategy used for the analysis is depicted (Fig. S5G).

\section{Adoptive transfer of OVA-Delayed Type Hypersensitivity (OVA-DTH) animals with CpG pulsed SMRT depleted cDC1 enhanced foot pad inflammation}

OVA induced Delayed Type Hypersensitivity (OVA-DTH) has been employed to manifest antigen specific T-cell response which is dependent on efficiency of antigen presentation by cells in host where T-cell experienced antigen initially in a sensitization phase and recall response in their challenge phase later on [27]. We asked whether SMRT deficient DCs can elicit strong inflammatory antigen specific $\mathrm{T}$ cells response in-vivo as compare to its counterpart control cDC1 cells. To address our hypothesis, we developed OVA-DTH mice model where we sensitize the mice with adjuvant emulsified OVA by subcutaneous (s.c) injection at day 0. Control and SMRT KD cDC1 pulsed with CpG and OVA were adoptively transferred at day 14. After one week, mice received OVA challenge locally in the left footpad to induce hypersensitivity mediated inflammation on day 20. Footpad inflammation was measured every $12 \mathrm{~h}$ till $72 \mathrm{~h}$ after OVA challenge. After $72 \mathrm{~h}$, the animals were dissected to assess the impact of DC treatment on T-cell immune-modulation (Fig. 5A). We found that treatment of SMRT depleted and activated cDC1 DCs in OVA sensitized animals showed profound and consistent increase in footpad inflammation till 72h (Fig. 5B and Fig. 
S6A). The T-cell subtypes in the popliteal and inguinal lymph nodes were also examined. We found a significantly increased Th1 subtype in lymph nodes in SMRT KD treated condition as compared to control group as marked by increased IFN- $\gamma$ and T-bet positive Tcell population (Fig. 5C). PBS treated animals were used in this study as control group. At the same time, we found a significantly higher Th17 T-cell population as evident from increased IL-17 and ROR $\gamma \mathrm{t}$ positive cells in mice injected with SMRT KD cDC1 (Fig. 5D). It has been reported that Th1 response induce tolerance by inhibiting Th17 response which was consistent in control cDC1 treated mice [27]. This phenomena was abolished in mice treated with SMRT KD cDC1 cells. Next, we asked the specificity of this observed T cell response by assaying the level of IgG subtypes in the sera of these mice collected at $0 \mathrm{~h}$ and $72 \mathrm{~h}$ from treated and control animals. We found that both mice group treated with OVA pulsed control and SMRT cDC1 showed induced OVA specific IgG subtypes as compare to mice treated with PBS. We did not find any difference between OVA pulsed control and SMRT KD cDC1 treated mice group (Fig. S6B). These results showed that decreased SMRT level in DCs generate a very strong inflammatory T cells response in the host.

\section{Adoptive transfer using CpG pulsed SMRT KD cDC1 regresses murine B16F10 melanoma tumor burden}

It has been reported widely that DC vaccine therapy induces tumor associated antigen (TAA)-specific oncolytic $\mathrm{CD}^{+}{ }^{+} \mathrm{T}$-cell activity [28]. Therefore, to assess the physiological impact of enhanced cytotoxic CD8 ${ }^{+} \mathrm{T}$-cell induced by SMRT depleted DCs, we developed a B16F10 melanoma model in C57BL/6 mice. The major objective was to check if SMRT KD cDC1 treatment could enhance and attract oncolytic $\mathrm{CD}^{+}{ }^{+} \mathrm{T}$-cell population in the developing tumor. First, we vaccinated the animals with B16F10 melanoma tumor antigen primed SMRT KD or control cDC1 to induce immunity against B16F10 tumors. We hypothesized that animals that received vaccination using inflammatory SMRT KD cDC1 loaded with B16 antigens could resist the increasing tumor burden compared to their control littermates. The vaccination with tumor antigen loaded SMRT KD or control cDC1 were done 10 days prior to subcutaneous injection of B16F10 cells for tumor development. To prime or load the control and SMRT KD cDC1 with B16F10 melanoma TAA we pulsed control and SMRT KD DCs with B16F10 tumor lysate at 1:1 ratio prepared from equal number of B16F10 melanoma cells. Pulsing with tumor lysate was performed overnight for TAA presentation on DCs. Next day the TAA loaded DCs were washed and pulsed with 
CpG for $2 \mathrm{~h}$ and $0.5 \times 10^{6}$ cells were injected per mice in the shaved left flank of the mice. Mice were given vaccination in the form of TAA loaded DCs 10 days prior and a booster dose was given 7 days prior to tumor injection. Tumor development was induced in these mice after 7 days by injecting $0.1 \times 10^{6}$ B16F10 cells subcutaneously in mice on the other flank (Fig. 5E). Tumor volumes were measured every alternate day starting from 7 days till 16 days post tumor initiation and we found that in SMRT cDC1 treated group the tumor burden was significantly reduced as compared to control groups (Fig. 5F, 5G and Fig. S6C). Further to assess the $\mathrm{CD}^{+}{ }^{+} \mathrm{T}$-cell cytotoxicity, we sacrificed the mice at 16 days post tumor initiation, dissected the tumors and made single cell suspension by collagenase treatment. The cells were then stimulated using PMA/Ionomycin/BFA for 5h, and $\mathrm{CD}^{+} \mathrm{CD}^{+} \mathrm{T}$-cells were analyzed. We found that frequency of Perforin, GrB and IFN- $\gamma$ percentage positive cells were significantly increased in SMRT KD cDC1 vaccinated animals as compared to other treatment groups (Fig. 5H and Fig. S6D, S6E). The tumors isolated from all the three groups of the animals showed a significantly decreased tumor weight in SMRT KD cDC1 vaccinated condition (Fig. 5I).

\section{Comparative genomics of NCoR1 and SMRT KD cDC1 showed differential control of STAT3 signaling mediated IL-10 regulation}

Recently, we reported that NCoR1 directly binds and strongly represses tolerogenic genes such as Il-10, Il-27, Cd83 and Socs3 in cDC1. NCoR1 depletion drastically increased the expression of these genes thereby leading to Treg generation ex vivo and in vivo [20]. Contrary to this, here we found that NCoR1 paralog SMRT depleted cDC1 demonstrated drastically increased inflammatory response in vitro, ex vivo and in vivo. Therefore, we performed comparative genomic analysis using global transcriptome and binding analysis (RNA-seq and ChIP-seq) to understand the differential gene regulation by NCoR1 and SMRT. We performed RNA-seq of SMRT KD and control cDC1 as well as ChIP-seq profiling of SMRT in wild type cDC1 line before and after 6h CpG stimulation. Differential gene expression analysis identified 957 and 813 genes up- and down-regulated genes respectively after 6h CpG stimulation (Log2 Fold change $>1$ and adjusted p-value $\leq 0.05$ ) in SMRT KD cDC1 as compared to control cells (Fig. 6A, Table S4). Using ChIP-seq analysis, we identified the target genes that were differentially expressed and bound by SMRT. We found that the number of directly bound SMRT genes were relatively higher than the down-regulated ones supporting its role as a co-repressor (Fig. 6B, Table S5). 
Ingenuity pathway analysis (IPA) of direct target differentially regulated genes in SMRT KD condition depicted up-regulation of inflammatory response pathways such as IL-12 signaling (Fig. 6C, Table S6). In contrast to NCoR1, SMRT KD gene list showed downregulation of IL-10 signaling at 6h CpG stimulation (Fig. 6D, Table S6). To further explore differential gene regulation by SMRT as compared to NCoR1, we did principal component analysis (PCA) of NCoR1 and SMRT KD cDC1 RNA-seq data and identified that SMRT KD unstimulated and 6h CpG stimulated condition cluster separately from respective NCoR1 samples (Fig. S7A). Interestingly, in unstimulated condition the number of differentially regulated genes (up-regulated $(n=771)$ and down-regulated $(n=612))$ are much higher in SMRT depleted condition as compared to NCoR1 KD DCs (Fig. 6E and Fig. S7B, Table S4). The number of up-regulated genes in SMRT KD 6h CpG were also relatively less as compared to NCoR1 KD 6h CpG condition. The PCA analysis and difference in the number of differentially expressed genes (DEGs) between SMRT and NCoR1 indicated towards a differential control of gene regulation by SMRT and NCoR1 in cDC1. To identify the differential gene expression patterns in NCoR1 and SMRT KD cDC1, we performed unsupervised K-means clustering of total DEGs based on log2 fold change and identified six clusters (Fig. 6F and Fig. S7C, Table S7). The genes in cluster-1 showed increased fold change in both NCoR1 and SMRT KD and depicted enriched pathways for “Interferon signaling”, “Activation of IRF by cytosolic pattern recognition receptors”, “IL12 signaling and production in macrophages”. On the contrary, cluster-2 genes showed differential regulation between SMRT and NCoR1 KD 6h CpG condition and pathways enriched were "Th2 pathway", "STAT3 pathway”, “IL-17-A signaling” and "IL-10 Signaling”. (Fig. 6G, Table S8). Cluster 3-6 genes were enriched for other cytokine signaling pathways terms such as “IL-8”, “IL-7” and “IL-15” signaling (Fig. S7C-7D, Table S8). These observations clearly suggested that inflammatory immunogenic response genes such as $I l-12 b$ and $I l-6$ are repressed by both NCoR1 and SMRT, however, the regulatory genes like Il-10, Socs3 are strongly repressed by NCoR1 only (Fig. 6H). Apart from the above listed gene we manually curated the DEGs and found multiple genes involved in generating pro-inflammatory response in SMRT KD cDC1. Few of them include upregulation in zbtb20 and downregulation in wnt11, clec4a2 in SMRT KD cDC1s (Table S4) [29-32]. As IL-10 is a physiologically important cytokine and well reported to perturb the inflammatory response towards immune-tolerance, understanding mechanistic control of differential IL-10 regulation by SMRT and NCoR1 was interesting [33]. We compared the genome wide binding of SMRT and NCoR1 before and after 6h CpG 
activation and found that the total number of bindings were quite similar ( 13,000 peaks) for both the factors (Fig. S7E). In SMRT, 40\% ( $\approx 5000)$ of the total bound sites are distributed at the promoter-proximal ( $\pm 1 \mathrm{~kb}$ to TSS) regions whereas only 18\% of NCoR1 peaks $(\approx 2000$ peaks) were found at promoter-proximal regions (Fig. S7F). Besides, classification of differential NCoR1 and SMRT peaks identified NCoR1 dominant $(12,473)$, NCoR1 and SMRT common (5949) and SMRT dominant (2707) bound genomic regions (Fig. 6I, 6J and Fig. S7G). NCoR1 and NCoR1-SMRT common or co-bound genomic regions are distributed in intronic or distal intergenic regions compared to SMRT dominant genomic regions which are predominantly present in promoter-proximal regions (Fig. 6I, 6J and Fig. S7G). This strongly suggested that unique SMRT binding sites mostly regulate genes through promoter-proximal binding whereas unique NCoR1 and NCoR1-SMRT common binding through far-distal enhancer regions. To identify the putative transcription factors (TFs) that undergo recruitment after $6 \mathrm{~h} \mathrm{CpG}$ activation at these co-repressors bound genomic regions, we performed de novo motif enrichment analysis and found that PU.1, RUNX2 and Jun-Fos/AP1 TFs motifs were enriched at almost all NCoR1 and SMRT bound genomic regions in unstimulated and $6 \mathrm{~h}$ CpG activated cells. Interestingly, NFkB motifs were found to be enriched at genomic regions where unique NCoR1, unique SMRT and NCoR1-SMRT common binding showed significant differential increase in ChIP-seq signal after 6h CpG activation. The IRF8 and IRF4 motifs were enriched at unique NCoR1 and NCoR1-SMRT co-bound regions in unstimulated and 6h CpG activation conditions respectively (Fig. 6K and Fig. S7H). The above enriched motifs corroborate with our previous report [21]. At the same time, we also found an enrichment of STAT3 motif at genomic regions where both NCoR1 and SMRT or only SMRT showed significant increase in ChIP-seq signal after 6h CpG stimulation. The genes annotated to unique NCoR1 CpG and NCoR1-SMRT CpG co-bound genomic regions were found to be enriched for "Th1 and Th2 pathway”, “Th-17 signaling pathway”, "NFkB signaling pathway”, and “JAKSTAT signaling pathway” (Fig. 6L and Fig. S7I, Table S9). Unique SMRT CpG bound genomic regions associated genes were enriched for terms such as "Chemokine signaling pathway" and "JAK-STAT signaling pathway" (Fig. 6L). Overall, the integrative genomics analysis predicted that STAT3-IL-10 signaling is differentially controlled by NCoR1 and SMRT. 
SMRT mediated down-regulation of NR4A1 inhibited mTOR-STAT3 signaling leading to IL-10 suppression

Next, we validated differentially enriched JAK-STAT signaling pathway. We found that the JAK-STAT signaling pathway associated genes were down-regulated in activated SMRT KD DCs whereas it was up-regulated in CpG activated NCoR1 KD condition (Fig. 7A, 7B). STAT3 TF plays a central role in Jak-Stat signaling and regulated expression of $I l-10$ and Socs3. Along with this we found down-regulation of Socs3 and other NFkB inhibitory genes such as Nfkbia and Tnfaip3 after 6h CpG stimulation in SMRT KD cDC1. First to confirm the differential regulation of p-STAT3 in NCoR1 and SMRT, we did western blotting for p-STAT3 in NCoR1 KD, SMRT KD and control cDC1 at 0h, 2h, and 6h after CpG activation. We found that p-STAT3 is significantly down-regulated in SMRT KD cDC1 compared to control cells whereas it was significantly upregulated in NCoR1 depleted DCs (Fig. 7C-7D). It is well reported that STAT3 binds to the $I l-10$ gene to regulate its expression [34]. We also checked STAT3 binding on Il-10 gene at $0 \mathrm{~h}$ and $6 \mathrm{~h}$ LPS stimulation in BMDCs (Fig. S7J) [35]. Therefore, we performed chromatin immunoprecipitation (ChIP) for p-STAT3 followed by RT-qPCR to infer the binding of pSTAT3 on Il-10 gene after 2h CpG challenge in control, NCoR1 KD, and SMRT KD cDC1. We observed reduction in p-STAT3 binding on Il-10 in SMRT KD DCs relative to control DCs whereas on the other side the binding was found to be enhanced in NCoR1 KD cells as compared to control cells (Fig. 7E). Moreover, to understand the upstream control of STAT3 signaling in these DCs we looked into the literature and identified that mTOR has been reported to control STAT3 activation and mTOR on the other hand is regulated by nuclear receptor NR4A1 also known as NURR-77 [36, 37]. We first checked the regulation of phospho-mTOR (p-mTOR) in NCoR1 and SMRT depleted cDC1 and found that pmTOR is upregulated in NCoR1 KD cDC1 whereas it is drastically reduced after SMRT depletion (Fig. 7F, 7G). In addition, we found that Nr4a1 is significantly down-regulated in unstimulated as well 6h CpG challenged SMRT depleted DCs (Fig. 7H). We also confirmed this observation by assessing the NR4A1 protein expression in SMRT depleted and control cDC1 before and after $2 \mathrm{~h}$ and $6 \mathrm{~h}$ CpG activation and found that NR4A1 is significantly down-regulated in SMRT KD DCs as compared to control cells (Fig. 7I, 7J). Further to confirm that NR4A1 indeed is controlling the STAT3 signaling through mTOR 


\section{Discussion}

Dendritic cells make a strong connecting link between innate and adaptive immunity. Besides a fine balance of DC signals is pertinent for development of an optimal immunogenic versus tolerogenic response to protect from autoimmune diseases and opportunistic infections. Though signaling pathways controlling one response versus another has been widely explored, how this balance is fine-tuned in DCs is interesting to understand for developing DC based therapies. Nuclear receptor co-repressors NCoR1 and SMRT have widely been reported as global transcriptional repressors [20,38] but their role in DCs is unexplored. We recently reported that NCoR1 directly binds and controls regulatory genes like $\mathrm{Il}-10, \mathrm{Cd} 274, \mathrm{Cd} 83$ and Il-27 and its loss of function in DCs enhanced Tregs development [21]. In contrast to this, here we found that NCoR1 paralog SMRT depletion induced strong inflammatory responses in cDC1 through enhanced Th1 and Th17 frequency along with increased cytotoxic T-cell activity. These findings depicted the role of these two co-regulators in fine-tuning inflammatory versus tolerogenic signals in cDC1 DCs. To understand this further, we performed comparative genomic analysis of NCoR1 
and SMRT depleted DCs and found that they differential regulated mTOR-STAT3 signaling pathway leading to tight regulation of IL-10. Overall, our analysis for the first time identified a fine switch that could be targeted to modulate inflammatory versus tolerogenic program in cDC1 DCs.

IL-10 is a potent anti-inflammatory cytokine that can limit host inflammatory response to pathogens thereby preventing host from damage. Dysregulated IL-10 is involved in enhanced immune-pathology and associated with development of auto-immune diseases[39]. The expression of IL-10 is dependent on STAT3 TF and it has been reported that STAT3 regulates $I l-10$ expression by binding to its regulatory region [40, 41]. At the same time, the positive feedback loop from IL-10 through STAT3 maintains its sustained expression. Immune-profiling analysis of NCoR1 and SMRT depleted cDC1 depicted significant upregulation of IL-10 in NCoR1, whereas it was drastically down-regulated in SMRT KD. When we looked at STAT3 regulation employing the integrative genomic analysis, it clearly showed significant down-regulation of STAT3 pathway in SMRT KD cDC1s. In contrast to this it was significantly upregulated in NCoR1 KD condition. Moreover, we found that there is increased binding of STAT3 on Il-10 in NCoR1 as compared to SMRT depleted cells. Overall, it is quite intriguing that two highly homologous co-regulators showed this differential regulation of an important pathway i.e., STAT3 and thereby IL-10 expression. Apart from STAT3 there are other transcription factors like Sp1/ Sp3, NF-kB, c-Maf, Smad4 that also exhibit a similar phenomenon in macrophages. However, Samarasinghe and colleagues showed that there is no increased binding of Sp1/Sp3 on Il-10 promoter in murine BMDCs [42]. We further explored to identify the mechanisms for differential regulation of STAT3 signaling. It has been reported that mTOR activates STAT3 [43] and we found that p-mTOR is up-regulated in activated NCoR1 KD cDC1 whereas it is drastically reduced in unstimulated as well as activated SMRT KD cDC1.

Furthermore, we observed that SMRT depleted DCs have sustained and increased expression of pro-inflammatory cytokines like IL-6, IL-12 and IL-23 thereby leading to enhanced development of Th1 and Th17 cells ex vivo and in vivo animal models. When we analyzed our global transcriptome data, we found that Socs3 is significantly downregulated along with negative regulators of NFkB signaling such as Nfkbia and Tnfaip3. It has been widely reported that SOCS3 depletion enhances the Th1 and Th17 cytokine release in DCs [44]. The Socs3 down-regulation was observed only in activated SMRT KD cDC1 after 6h 
activation, which suggested that the initial events of IL-10 and STAT3 decrease through down-regulation of mTOR activity are somehow leading to SOCS3 decrease. To our surprise, we found that SOCS3 and STAT3 both are down regulated in SMRT KD cDC1, as in several reports it has been documented that decreased SOCS3 favours enhanced STAT3 [45]. We hypothesize that it could be due to the dynamic time dependent regulation of STAT3 and SOCS3 in CpG activated SMRT KD cells.

Moreover, as we observed a drastic decrease of p-mTOR in unstimulated SMRT KD cDC1s, we looked into the regulators of mTOR. We found an interesting nuclear receptor Nr4a1 to be significantly down-regulated in RNA-seq data of unstimulated SMRT depleted DCs and even after CpG activation. NR4A1 has been reported to positively regulate mTOR activity and thereby STAT3 phosphorylation and IL-10 expression [46]. It is also shown that deficiency of NR4A1, a member of the nuclear receptor superfamily, leads to enhanced production of IL-6, TNF- $\alpha$, and IL-12 in both human and murine dendritic cells [18]. Similarly, peroxisome proliferator-activated receptor (PPAR- $\alpha$ ) also exerts antiinflammatory effects on monocytes and macrophages [47]. We showed that indeed NR4A1 expression was down-regulated supporting our finding in SMRT depleted DCs. On the other side complementing Nr4a1 in SMRT depleted cDC1 by treating with 6-MP rescued Il-10 expression. These indicated towards the contrasting inflammatory versus tolerogenic phenotype expressed by SMRT and NCoR1 depleted DCs.

In an in vivo physiological system, the maintenance of Th1, Th17, and Th2 balance depends on a number of factors including antigen presentation by MHC-II, co-stimulation and differential cytokine production [48]. To address whether the manipulation of DCs by SMRT KD towards an enhanced Th1 and Th17 type responses could deliver a long term functional and effector memory response, we developed DTH and B16F10 melanoma models. The SMRT depleted and control DCs were adoptively transferred and perturbation in DTH responses and B16F10 melanoma progression was observed. We found that SMRT depleted DCs have potential to enhance Th1 and Th17 type responses and thereby DTH enhancement in animals. At the same time, oncolytic CD8 ${ }^{+} \mathrm{T}$-cell activity was enhanced leading to reduced tumor burden in animals. Furthermore, it has been widely reported that in autoimmune diseases such as RA and Multiple Sclerosis (MS) enhanced Th1 and Th17 cells result in inflammatory symptoms [49]. As we observed enhanced Th1 and Th17 response by SMRT depleted DCs we found significantly reduced SMRT expression in mononuclear cells of RA patients as compared to controls. Therefore, it is further 
interesting to explore if SMRT co-repressor has some potential association with autoimmune pathogenesis and can be used as a target for immunotherapy.

\section{Materials and Methods}

Mice

C57BL/6 wild type mice bred and maintained at ILS animal facility. OT-II and OT-I transgenic mice (gifted by Prof. Hans Acha-orbea, University of Laussane) and C57BL6 Flt3 transgenic mice (gifted by Ton Rolink) were transported from SWISS. All the animal experiments were performed after getting due approval from the institutional animal ethics committee.

\section{Dendritic Cell (DC) Culture}

The CD8 $\alpha+$ mutuDC cell line we have worked was gifted by Prof. Hans Acha-Orbea's group [22]. The cell lines were maintained in culture at $37^{\circ} \mathrm{C}$ in a humidified incubator with 5\% CO2. Cell were cultured in complete IMDM-glutamax medium with all buffered conditions as reported previously.

For in vitro experiments, the DCs were plated in 12- or 6-well plates at a density of $5 \times 10^{5}$ or $1 \times 10^{6}$ cells $/ \mathrm{ml}$ overnight. The cells were then challenged with different activation media containing TLR9 agonist CpG-B, TLR3 agonist pIC for 2, 6 or 12 h. For performing RT-qPCR analysis the cells were washed in the plate once with PBS followed by addition of RNA-later (LBP) lysis buffer for lysis of cells. The plates were then stored at $-80^{\circ} \mathrm{C}$ until further RNA isolation and processing of samples.

\section{Generation of Stable SMRT KD CD8a $\alpha^{+}$MutuDCs}

For generating stable SMRT knockdown and their comparative control DC, lentiviral vector pLKO.1 (Sigma) containing three different sigma mission shRNA for Ncor2/Smrt were picked targeting chromosome 5 on mouse genome against exons 48, 19, and 14 respectively (Table S2). Viral particles packaged with shRNA expressing transfer plasmids were produced in $293 \mathrm{~T}$ cells using Cal-Phos (CaPO4) mammalian transfection kit according to an optimized protocol. We used a $2^{\text {nd }}$ generation lentiviral system which included PCMVR and PMD2G as packaging and envelope plasmids respectively. Human embryonic kidney (HEK) 293T cells were transfected with transfer plasmids containing three different Smrt shRNAs or control shRNAs along with pCMVR8.74 and pMD2G. 
After 12-14 h the culture medium was replenished and supernatant containing viral particles were collected after $24 \mathrm{~h}$ in $50 \mathrm{ml}$ conical tubes. Viral particle-containing culture supernatant was concentrated using ultracentrifugation at $50,000 \mathrm{~g}$ at $16^{\circ} \mathrm{C}$ for $2 \mathrm{~h}$ and preserved at $-80^{\circ} \mathrm{C}$ in small aliquots. For transduction of shRNA containing viruses in CD8 $\alpha^{+}$cDC1 MutuDC lines, the cells were plated at a density of $1.5 \times 10^{5}$ cells/well of 12 well plate followed by transduction with virus particles containing supernatant. The media was replaced with fresh media after $12 \mathrm{~h}$ of virus incubation with DCs followed by addition of $1 \mu \mathrm{g} / \mathrm{ml}$ puromycin selection medium after $72 \mathrm{~h}$ of media replacement for stable KD cells.

\section{RNA Isolation and RT-qPCR}

The extraction of RNA was done using NucleoSpin RNA Plus miniprep kit (Machery Nagel). Briefly cells were preserved in LBP lysis buffer in $-80^{\circ} \mathrm{C}$ and thawed by placing the plates/tubes on ice. Total RNA was isolated according to manufacturer's protocol. RNA concentration was estimated by nanodrop (Thermo) and then 1-2 $\mu \mathrm{g}$ of total RNA was used to prepare cDNA using high-capacity cDNA Reverse Transcriptase kit (Applied Biosystems). Quantitative PCR was performed using SYBR Green master (Roche) and PCR amplification was monitored in real-time using LightCycler-480 Instrument. Primer oligonucleotides for qPCR were designed using universal probe library assay design system and the primer pairs used are listed in Table S3. Primers were optimized for linear and single product amplification by performing standard curve assays.

\section{Flow Cytometry (FACS)}

We performed flow cytometry analysis using the well-established surface and intracellular (IC) staining protocols [21]. $5 \times 10^{5}$ and $1.5 \times 10^{6}$ cells were seeded for surface and IC staining respectively. Cells were either left unstimulated or stimulated with CpG or pIC for 6h. For staining the cells were dissociated and washed with FACS buffer (3\% FCS in $1 \mathrm{X}$ PBS, 5 mM EDTA). After washing, fluorochrome conjugated antibodies for proteins of interest were added to the cells as a cocktail in staining buffer (Table S1). For surface staining cells were stained in FACS buffer for $30 \mathrm{~min}$ in dark at $4^{\circ} \mathrm{C}$. For IC staining of cytokines the cells were first fixed with $2 \%$ paraformaldehyde for 20 min followed by permeabilization using $1 \mathrm{x}$ permeabilization buffer (eBiosciences). The fixed and permeabilized cells were then resuspended in IC staining buffer and stained with 
fluorochrome tagged antibodies for selected cytokines. For optimal staining the cells were incubated with antibodies for $30 \mathrm{~min}$ in dark. After incubation the cells were washed twice with FACS wash buffer and then acquired for differential expression analysis using LSRII fortessa flow cytometer (BD Biosciences). The acquired data was analyzed using FlowJoX software (Treestar). Antibodies used for flow cytometry experiments are listed in Table S1.

\section{Bio-Plex Assay for Cytokine Quantitation from Cell Culture Supernatants}

Bio-Plex assay (multiplex ELISA) was used to estimate the cytokine levels secreted in the cell culture supernatants of SMRT KD and control DC and BMcDC1 after $6 \mathrm{~h}$ of CpG stimulation according to previous reports [21]. After culture, the supernatants were stored at $-80^{\circ} \mathrm{C}$ in small aliquots until analysis. Cytokine levels were estimated using 23-plexmouse cytokine assay kit following the vendor recommended protocol (Biorad).

\section{Generation of Bone Marrow Derived DCs (BMDCs) for ex vivo Studies}

Six to eight-week-old female C57BL/6 mice were killed by cervical dislocation and disinfected using 75\% ethanol [21]. In short the tibias and femurs were removed under sterile conditions, then soaked in RPMI-1640 medium supplemented with 10\% FBS. Cells from both ends of the bone were flushed out with a needle of 1-mL syringe from the bone cavity into a sterile culture dish with RPMI-1640 medium. The cell suspension in the dish was collected and centrifuged at $350 \mathrm{~g}$ for $5 \mathrm{~min}$, and the supernatant was discarded. The cell pellet was suspended with 1x RBC lysis buffer (Tonbo) for 5-10 min on ice. Cell clumps were then passed through a $70 \mu \mathrm{m}$ strainer to obtain single cell suspensions. The lysed cells were washed once with RPMI-1640, counted and used for differentiation into DCs.

We followed a well-established protocol for differentiation of BMDCs with slight modifications. The cells, suspended in RPMI-1640 medium supplemented with $10 \%$ FBS, were distributed into 6 -well plates at a density of $1 \times 10^{6} \mathrm{cell} / \mathrm{ml} /$ well. Subsequently, $1 \mu \mathrm{l} / \mathrm{ml}$ of FLT3L containing sera was added into the medium. The cells were cultured at $37^{\circ} \mathrm{C}$ in an incubator containing 5\% CO2 and left untouched for 5 days. On day 5, the suspended and loosely attached cells were collected. 
The cells were plated into 96-well plate for lentiviral transduction using concentrated viruses at a density of $0.4 \times 10^{6}$ cells/well for each Smrt shRNA and control shRNA. After $72 \mathrm{~h}$ the cells were stimulated with $\mathrm{CpG}$ for $6 \mathrm{~h}$ and then immune-profiling was performed using flow cytometry.

\section{Co-culture of DCs with $\mathrm{CD4}^{+} \mathrm{T}$-Cells and $\mathrm{CD8}^{+}$T-cells for Assessing T-Cell Proliferation and Differentiation}

DC-T-cell co-culture experiments were performed according to well established protocol [50, 51]. Naïve $C D 4^{+}$or $C D 8^{+}$T-cells were purified from spleen of TCR-transgenic OT-II or OT-I mice using $\mathrm{CD}^{+}$or $\mathrm{CD} 8^{+} \mathrm{T}$-cell isolation kit. SMRT KD and control $\mathrm{CD} 8 \alpha^{+} \mathrm{CDC} 1$ DCs were seeded at a density of 10,000 cells/well in round bottom 96 well plates followed by pulsing with OVA peptide (323-339) or OVA peptide (257-264) overnight. Further DCs were stimulated with $\mathrm{CpG}$ or pIC for $2 \mathrm{~h}$. After $2 \mathrm{~h}$, purified OT-II or OT-I T-cells were added at the density of 100,000 cells/well (1:10 ratio). Then T-cell proliferation and differentiation into distinct Th subtypes Th1, Th2, Th17 and Tregs, in case of OT-II, and cytotoxic T-cells, in case of OT-I, were analyzed by FACS. Proliferation was measured using an amine based dye (eFluor 670). The rate of T-cell proliferation was inversely proportional to the median fluorescence intensity (MFI) measured in FACS after 72h of co-culture. For Th and cytotoxic T-cell differentiation profiling after 96h, the co-cultured T-cells were re-stimulated with PMA (10 $\mathrm{ng} / \mathrm{mL})$ and ionomycin $(500 \mathrm{ng} / \mathrm{mL})$ and followed by Brefeldin-A $(10 \mu \mathrm{g} / \mathrm{mL})$ treatment for $5 \mathrm{~h}$ to block the IC cytokines from being secreted. After 5 h, fluorochrome conjugated antibodies specific to different T-cell subtypes were used to profile T-cells into Th1 (T-bet and IFN- $\gamma$ ), Th2 (GATA3, IL-13), Tregs (CD25, FoxP3, IL-10) and Th17 (ROR $\gamma$ T, IL-17) or cytotoxic T-cells (perforin, IFN- $\gamma$, Granzyme-B). For gating effector T-cells we used CD44 as a marker (Table S1).

\section{Chromatin Immuno-Precipitation (ChIP) for p-STAT3}

The ChIP for p-STAT3 was performed according to the methods optimized previously by Raghav and Meyer's lab. For ChIP assays, 40 x $10^{6}$ CD8a + cDC1 MutuDCs were seeded in $15 \mathrm{~cm}^{2}$ plates and prepared for four ChIP assays by 10 min cross-linking with $1 \%$ formaldehyde (sigma) at room temperature followed by quenching using $2.5 \mathrm{M}$ glycine (sigma) for $10 \mathrm{~min}$. The plates were placed on ice and the cells were scraped and collected in $50 \mathrm{ml}$ conical tubes. The cells were then washed three times using cold 1x PBS at 2,000 rpm for $10 \mathrm{~min}$ at $4^{\circ} \mathrm{C}$ and the cell pellets were stored at $-80^{\circ} \mathrm{C}$. At the day of the ChIP 
experiment, the cells were thawed on ice followed by lysis using Farham lysis buffer (5 mM PIPES pH 8.0, 85mM KCl, 0.5\% NP-40 supplemented with protease and phosphatase inhibitors (Roche)) made in miliQ. The supernatant was aspirated and the pellet was resuspended in RIPA buffer (1\% NP-40, 0.5\% sodium deoxycholate, $0.1 \%$ SDS supplemented with Roche protease and phosphatase inhibitor tablet just before use). The chromatin was fragmented using a Bioruptor (Diagenode) sonicator for 30 min using high amplitude and 30s ON \& 30s OFF cycles to obtain 200-500 bp size fragments. A cooling unit was used to circulate the cold water during sonication to avoid de-crosslinking because of overheating. After sonication, chromatin length was checked in agarose gel. The fragmented chromatin was centrifuged at 10,000 rpm for 5 min and then clear supernatant was collected in $15 \mathrm{ml}$ conical tubes. The DNA concentration of the chromatin was estimated using a Nano-Drop (Thermo) and the chromatin was diluted with RIPA buffer to use $150 \mu \mathrm{g} / \mathrm{ml}$ of chromatin for each IP. M-280 sheep anti-rabbit IgG dynabeads 40ul/IP was taken in a $1.5 \mathrm{ml}$ MCT tube. 1ml RIPA buffer was added to the beads and placed on magnetic stand. The MCTs were inverted 5 times and allowed to stand for 3 min. The beads were washed in the same way 3 times. After the 3rd wash the beads were centrifuged shortly and the remaining RIPA buffer was aspirated. To the beads, $5 \mu l$ of mouse monoclonal anti-p-STAT3 (CST) was added to immunoprecipitated the chromatin complex at $4^{\circ} \mathrm{C}$ for $8 \mathrm{~h}$ on rocker shaker. After $8 \mathrm{~h}$ incubation, the beads were again placed on magnetic stand and washed with RIPA to get rid of unbound antibody. Chromatin was added to the beads and placed on a rotating rocker at $4^{\circ} \mathrm{C}$ overnight. Next day the tubes containing chromatin, antibody, and beads were taken out, placed on a magnetic stand and supernatant was aspirated. The beads were washed 5 times with LiCL IP wash buffer (100mM Tris pH7.5, 500mM LiCl, 1\% NP-40, 1\% sodium deoxycholate in miliQ) and 2 times with TE buffer (10mM Tris pH7.5, 0.1mM EDTA pH8 in miliQ). After removing the wash buffer completely, protein-bound chromatin complexes were eluted from beads using elution buffer (1\% SDS, 0.1M NaHCO3 in milli-Q water). The chromatin was incubated at room temperature for $30 \mathrm{~min}$ in elution buffer. A short spin was given and the MCT was again placed on magnetic stand to collect the eluted chromatin. The eluted chromatin was then reverse crosslinked by incubating the eluted supernatant at $65^{\circ} \mathrm{C}$ overnight on a heat block after adding $8 \mu \mathrm{l}$ of $5 \mathrm{M} \mathrm{NaCl}$. Next day DNA was purified from the reverse cross-linked chromatin by proteinase-K and RNase digestion followed by purification using PCR purification kit (Qiagen). The purified DNA was eluted in $40 \mu \mathrm{l}$ of elution buffer. 


\section{Chromatin Immuno-Precipitation (ChIP) for SMRT}

The ChIP for SMRT was performed according to the methods optimized previously by Raghav and Deplancke's lab [21, 38]. In short the cells were lysed in nuclei extraction buffer for $10 \mathrm{~min}$ at $4^{\circ} \mathrm{C}$ while shaking to isolate the nuclei. The isolated nuclei were then washed using protein extraction buffer at room temperature for $10 \mathrm{~min}$. Washed nuclei were resuspended in chromatin extraction and incubated for 20 min on ice. The chromatin was fragmented using a Bioruptor (Diagenode) sonicator to obtain 200-500 bp-sized fragments. The fragmented chromatin was centrifuged at 17,000g for $10 \mathrm{~min}$ and then clear supernatant was collected in chilled $15 \mathrm{ml}$ falcon tubes. The DNA concentration of the chromatin was estimated using a NanoDrop and the sonicated chromatin was diluted with ChIP dilution buffer to get $100 \mu \mathrm{g} / \mathrm{ml}$ of chromatin for each IP. BSA and ssDNA (Salmon Sperm DNA) -preblocked protein-A sepharose ( $80 \mu \mathrm{l} / \mathrm{IP})$ beads were added to the samples and incubated for $2 \mathrm{~h}$ to remove non-specific- binding chromatin. To the supernatant, 5 $\mu \mathrm{l} / \mathrm{IP}$ rabbit polyclonal anti-SMRT antibody (Abcam) was added to immuno-precipitate the chromatin complex at $4^{\circ} \mathrm{C}$ overnight. After the overnight incubation, $50 \mu$ l blocked beads were added to each sample and incubated for $90 \mathrm{~min}$ at $4^{\circ} \mathrm{C}$ to pull down the respective antibody-chromatin complexes. The beads were then washed four times with low salt wash buffer followed by two washes with high salt wash buffer, lithium chloride wash buffer and tris-EDTA (TE) buffer. After removing the wash buffer completely, protein-bound chromatin complexes were eluted from beads for 30 min using elution buffer. The eluted chromatin was then reverse-crosslinked by incubating the eluted supernatant at $65^{\circ} \mathrm{C}$ overnight on a heat block after adding $8 \mu \mathrm{l}$ of $5 \mathrm{M} \mathrm{NaCl}$. The next day, DNA was purified from the reverse crosslinked chromatin by proteinase and RNase digestion followed by purification using Qiagen DNA purification columns. The purified DNA was eluted in 50 $\mu \mathrm{l}$ of Qiagen elution buffer.

\section{ChIP/RNA-seq library preparation for sequencing}

For RNA-seq library preparation 2ug of total RNA was used to isolate mRNA using magnetic beads with mRNA isolation kit (PolyA mRNA isolation module, NEB). Later mRNA library preparation kit, NEB was used for RNA-seq library preparation according to manufacturer's protocol. Concentration of the libraries were estimated by Qubit 2.0 (Invitrogen) and fragment sizes were analysed in Bio-analyzer (Agilent). The libraries were then sequenced on Illumina NextSeq 550 platform. 
Similarly for ChIP seq $30 \mu$ l ChIP-DNA was processed for library preparation according to ChIP-seq library preparation protocol (NEB) [21]. After library preparation and quality check, the libraries were sent to NGS service provider (Sci Genome, Bangalore, India) for Illumina sequencing using NextSeq-550 instrument.

\section{Western Blotting}

Cells were collected in RIPA buffer (0.5 M EDTA, 1 M Tris-Cl pH7.5, 1 M NaCl, 200 $\mathrm{mM}$, Roche protease inhibitor) at $0 \mathrm{~h}, 2 \mathrm{~h}$ and $6 \mathrm{~h}$ CpG stimulation. Cells were lysed completely by sonicating the samples in Bioruptor (Diagenode) for 10 min using high amplitude and 30s ON \& 30s OFF cycles. Protein concentrations were measured in 96 well plate using BCA protein assay kit (BioRad).

\section{Delayed Type Hypersensitivity (DTH) Assay}

DTH was performed using culture grade ovalbumin (OVA) from chicken egg (Sigma) dissolved in 1x PBS at a concentration of $1 \mathrm{mg} / \mathrm{ml}$ and filtered through 0.2-micron PES syringe filter. $1.5 \mathrm{ml}$ alum and $1.5 \mathrm{ml}$ OVA was added in a glass beaker and passed through a glass syringe multiple times to make an emulsion. $300 \mu l$ per mice was injected subcutaneously in the back behind ears in each mice for OVA immunisation. After 14 days control and SMRT KD DCs were pulsed with OVA (100ug/ml) for $4 \mathrm{~h}$. Cells were then stimulated with CpG. After $2 \mathrm{~h}$ of stimulation the cells were dissociated and injected at 10 x $10^{6}$ cells/mice. Further after 7 days OVA $(20 \mathrm{mg} / \mathrm{ml})$ was heated at $80^{\circ} \mathrm{C}$ for $2 \mathrm{~h}$, cooled, and injected in foot pad of mice $(25 \mu \mathrm{l} /$ mice). 1x PBS was injected in the alternative footpad. Paw thickness was measured till 72h using Vernier caliper. After 72h the popliteal and inguinal lymph nodes were isolated and checked for T-bet IFN- $\gamma$ as well as ROR $\gamma \mathrm{t}$ IL17.

\section{OVA specific ELISA}

To examine OVA specific immune response we performed experiments as described [21, 52]. In brief, we collected sera at day 20 and day 23 after OVA immunization to perform ELISA for OVA specific IgG titer. Elisa plates were coated with 100ug/ml of OVA (Sigma) prepared in coating buffer $\left(\mathrm{Na}_{2} \mathrm{CO}_{3}, \mathrm{NaHCO}_{3}\right.$, Sodium Azide) overnight at $4^{\circ} \mathrm{C}$ following five washes with washing buffer (PBS with 0.05\% tween -20). Blocking was done with PBST containing $0.5 \%$ gelatin for $1 \mathrm{~h}$ at $37^{\circ} \mathrm{C}$. After five times washing, $50 \mu \mathrm{l}$ diluted sera were added from mice and kept for $1.5 \mathrm{~h}$ at $37^{\circ} \mathrm{C}$. IgG1 (dilution 1:10,000) and 
IgG2a (dilution 1:100) was detected using biotin labelled anti-mouse IgG1 and IgG2a while total IgG (dilution 1:1000) was detected using anti-mouse HRP conjugated IgG followed by anti-mouse streptavidin-HRP (Biolegend). The plates were further incubated at $37^{\circ} \mathrm{C}$ for $1 \mathrm{~h}$ and washed 7 times with washing buffer. Color was developed by TMB

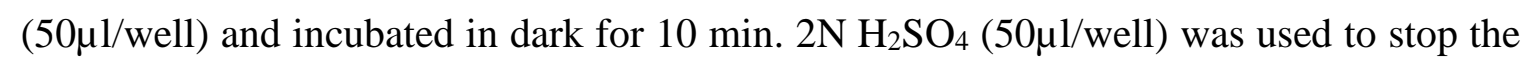
reaction. The plates were read using ELISA reader for IgG estimation at $450 \mathrm{~nm}$.

\section{Tumor cell lysate preparation}

Tumor lysate was prepared from previous reports with some modifications [53]. B16/F10 tumor cells were adjusted to $3 \times 10^{6}$ cells/ml in DMEM medium. Cells were subjected to 3 freeze $\left(-80^{\circ} \mathrm{C}\right) /$ thaw $\left(40^{\circ} \mathrm{C}\right)$ cycles of minimum $20 \mathrm{~min}$ each. The lysed cells were checked under trypan blue staining and centrifuged at 12,000rpm for $15 \mathrm{~min}$. The supernatant was passed through a $40 \mu \mathrm{m}$ cell strainer before adding to cDC1s seeded at a density of $3 \times 10^{6}$ (DC:tumor cell ratio of 1:1).

\section{B16F10 Tumor Model}

Mice were injected subcutaneously (s.c.) with $0.5 \times 10^{6}$ tumor cells into the left flank, and a booster dose was re-injected 3 days later. 7 days after booster dose, $0.1 \times 10^{6}$ cells were injected subcutaneously in mice in the right flank. Tumor growth was measured every day using vernier caliper till 16 days. Tumors were removed, weighed, and dissociated to make single cell suspensions.

\section{Isolation of tumor and tumor re-stimulation}

For IC perforin, granzyme-B, and IFN- $\gamma$ staining, single cell suspensions were $e x$ vivo restimulated with $10 \mathrm{ng} / \mathrm{ml}$ PMA, 500ng/ml ionomycin, and $5 \mu \mathrm{g} / \mathrm{mL}$ Brefeldin A for $5 \mathrm{~h}$. Cells were labelled with indicated surface-staining antibodies, fixed with $2 \%$ PFA, permeabilized with permeabilization buffer and stained with IC antibodies.

\section{RNAseq data processing and analysis}

Raw reads of SMRT KD RNA-seq samples and its matched control in unstimulated and 6h CpG stimulation were checked for quality using FASTQC [54] , and aligned to mouse genome (RefSeq mm10) using hista2 [55] (with default parameter). Similarly, raw reads of NCoR1 KD and its matched control RNAseq were processed for quality control and 
alignment. Raw counts of genes were extracted using featureCount (featureCounts -p -B) [56]. Principal component analysis was performed on variance stabilized transformation values from DESeq2[57] using the plotPCA function and plotted using ggplot2 [58]. Further, differential gene expression analysis was performed between NCoR1/SMRT KD compared to its matched control in unstimulated and 6h CpG stimulation condition. Genes were filtered based on log2foldchange (upregulated $>=1$ and downregulated $<=-1$ ) and adjusted P-value $(<0.05)$. Total differentially expressed genes were combined from all the comparison and unsupervised k-means clustering were performed based on log2foldchange values and divided into six clusters. Pathway enrichment analysis for each cluster was performed using Ingenuity pathway analysis.

\section{ChIP-seq data processing and analysis}

SMRT ChIP-seq raw reads in unstimulated and 6h CpG stimulation were checked for quality using FASTQC and aligned to mouse genome (RefSeq mm10) using bowtie2 [59]. Reads were filtered using MarkDuplicates function of Picard and mapping quality $>=10$ using samtools [60, 61]. Peak calling was performed using Homer findPeaks using factor as style. Peak calling for NCoR1 ChIP-seq data from our previous study were performed with reads down sampled to the level of SMRT i.e 9M. Distribution analysis of peaks based on distance relative to TSS were performed using ChIPSeeker [62]. Peak from both NCoR1 and SMRT were merged using bedops merge command and consensus peaks were generated for further downstream analysis[63]. GeneOverlap R package were used to identify differentially expressed genes that are direct target of SMRT[64].

\section{ChIP-seq Peak analysis}

Differential binding analysis for NCoR1 and SMRT were carried out on the merged peak using the getDifferentialPeaks program of Homer with cut-off of 2-fold enrichment over background [65]. Peaks were filtered out that didn't show any differential binding in any of the comparison. Differential peaks were then categorized based on fold change. Peaks from different categories were annotated to nearest genes using the ChIPseeker R package [62]. De novo transcription factor motif enrichment analysis was performed using findMotifs.pl (size -50, 50 -len 8, 10, 12) using background generated from provided input genomic regions. P-value $+<=1 \mathrm{e}-10$ were used to filter significantly enriched TF motifs. 
KEGG pathway enrichment analysis of differentially expressed genes associated with each binding category were performed using clusterProfiler R package[66].

\section{Acknowledgments}

General: We are sincerely thankful to Hans Acha-Orbea for providing us the DC cell lines, OT-I, OT-II, and FLT-3 transgenic mice. We would like to thank Subhasish Prusty for helping in western experiments and Niyati Das for helping in animal experiments. A.J. is supported by ILS fellowship, A.A. is supported by DBT-SRF, G.P.M. is supported by DBT BINC fellowship, K.S. is supported by UGC-SRF, S.S. is supported by ILS fellowship, M.A. is supported by CSIR funding, V.K.B. is supported by ILS tribal flagship fellowship, A.T. is supported by DST INSPIRE.

Funding: This study is supported by grants from SERB (EMR/2016/000717, CRG/2019/005893), DST-SNSF (DST/INT/SWISS/SNSF/P-47/2015), DBT Ramalingaswami fellowship, DBT (BT/PR15908/MED/12/725/2016). ILS provided intramural support and infrastructure.

Author contributions: AJ, AA, GPM, BG, HAO, SBS and SKR conceptualized the study, AJ, AA, KS, SS, AT, VKB, and APM were involved in performing experiments and interpretation of the results. GPM and SKR did all the computational analysis of the genomics data. AJ, AA, GPM and SKR wrote the manuscript. Funding for the study was secured by SKR from different funding agencies acknowledged in funding section.

Competing interests: Authors declare no competing interest.

Data and materials availability: RNA-seq and ChIP-seq data of SMRT samples has been submitted to ArrayExpress and available under accession number E-MTAB-10070 (http://www.ebi.ac.uk/arrayexpress/experiments/E-MTAB-10070, username: Reviewer_EMTAB-10070, password: wnYYTKCB) and E-MTAB-10069 (http://www.ebi.ac.uk/arrayexpress/experiments/E-MTAB-10069, username: Reviewer_EMTAB-10069, password: 0112Uknx) respectively. Username and password for the accessibility is sunilraghav@ils.res.in and 86fx4RKH respectively. RNA-seq and ChIP-seq data of NCoR1 samples are available at Gene Expression Omnibus having accession 
number GSE110423. The $\mathrm{R}$ code used in this study has been deposited to https://github.com/sraghav-lab/NCoR1-and-SMRT-Project.

\section{References}

1. $\quad$ Steinman, R.M., Dendritic cells: understanding immunogenicity. Eur J Immunol, 2007. 37 Suppl 1: p. S53-60.

2. Steinman, R.M., D. Hawiger, and M.C. Nussenzweig, Tolerogenic dendritic cells. Annu Rev Immunol, 2003. 21: p. 685-711.

3. Hilligan, K.L. and F. Ronchese, Antigen presentation by dendritic cells and their instruction of CD4+ T helper cell responses. Cell Mol Immunol, 2020. 17(6): p. 587-599.

4. Manicassamy, S. and B. Pulendran, Dendritic cell control of tolerogenic responses. Immunol Rev, 2011. 241(1): p. 206-27.

5. Abou Fakher, F.H., et al., TLR9-dependent activation of dendritic cells by DNA from Leishmania major favors Th1 cell development and the resolution of lesions. J Immunol, 2009. 182(3): p. 1386-96.

6. Schnorrer, P., et al., The dominant role of $\mathrm{CD} 8+$ dendritic cells in cross-presentation is not dictated by antigen capture. Proc Natl Acad Sci U S A, 2006. 103(28): p. 10729-34.

7. Musumeci, A., et al., What Makes a pDC: Recent Advances in Understanding Plasmacytoid DC Development and Heterogeneity. Front Immunol, 2019. 10: p. 1222.

8. Wculek, S.K., et al., Dendritic cells in cancer immunology and immunotherapy. Nat Rev Immunol, 2020. 20(1): p. 7-24.

9. Kawasaki, T. and T. Kawai, Toll-like receptor signaling pathways. Front Immunol, 2014. 5: p. 461.

10. Della Mina, E., et al., Inherited human IRAK-1 deficiency selectively impairs TLR signaling in fibroblasts. Proc Natl Acad Sci U S A, 2017. 114(4): p. E514-E523.

11. Kawai, T. and S. Akira, Signaling to NF-kappaB by Toll-like receptors. Trends Mol Med, 2007. 13(11): p. 460-9.

12. Kimura, A., et al., Suppressor of cytokine signaling-1 selectively inhibits LPS-induced IL-6 production by regulating JAK-STAT. Proc Natl Acad Sci U S A, 2005. 102(47): p. 1708994.

13. Chaudhry, A., et al., Interleukin-10 signaling in regulatory $T$ cells is required for suppression of Th17 cell-mediated inflammation. Immunity, 2011. 34(4): p. 566-78.

14. Maroof, A., et al., Posttranscriptional regulation of II10 gene expression allows natural killer cells to express immunoregulatory function. Immunity, 2008. 29(2): p. 295-305.

15. Sallusto, F. and A. Lanzavecchia, The instructive role of dendritic cells on T-cell responses. Arthritis Res, 2002. 4 Suppl 3: p. S127-32.

16. Audiger, C., et al., The Importance of Dendritic Cells in Maintaining Immune Tolerance. J Immunol, 2017. 198(6): p. 2223-2231.

17. Nencioni, A., et al., Dendritic cell immunogenicity is regulated by peroxisome proliferatoractivated receptor gamma. J Immunol, 2002. 169(3): p. 1228-35.

18. Tel-Karthaus, N., et al., Nuclear Receptor Nur77 Deficiency Alters Dendritic Cell Function. Front Immunol, 2018. 9: p. 1797.

19. Jepsen, K. and M.G. Rosenfeld, Biological roles and mechanistic actions of co-repressor complexes. J Cell Sci, 2002. 115(Pt 4): p. 689-98. 
20. Ghisletti, S., et al., Cooperative NCoR/SMRT interactions establish a corepressor-based strategy for integration of inflammatory and anti-inflammatory signaling pathways. Genes Dev, 2009. 23(6): p. 681-93.

21. Ahad, A., et al., NCoR1: Putting the Brakes on the Dendritic Cell Immune Tolerance. iScience, 2019. 19: p. 996-1011.

22. Fuertes Marraco, S.A., et al., Novel murine dendritic cell lines: a powerful auxiliary tool for dendritic cell research. Front Immunol, 2012. 3: p. 331.

23. Nish, S.A., et al., $T$ cell-intrinsic role of IL-6 signaling in primary and memory responses. Elife, 2014. 3: p. e01949.

24. Maloy, K.J. and M.C. Kullberg, IL-23 and Th17 cytokines in intestinal homeostasis. Mucosal Immunol, 2008. 1(5): p. 339-49.

25. Alzabin, S. and R.O. Williams, Effector T cells in rheumatoid arthritis: lessons from animal models. FEBS Lett, 2011. 585(23): p. 3649-59.

26. Hemdan, N.Y., et al., Interleukin-17-producing Thelper cells in autoimmunity. Autoimmun Rev, 2010. 9(11): p. 785-92.

27. Utku, N., et al., TIRC7 deficiency causes in vitro and in vivo augmentation of $T$ and $B$ cell activation and cytokine response. J Immunol, 2004. 173(4): p. 2342-52.

28. Kalinski, P., et al., Dendritic cell-based therapeutic cancer vaccines: what we have and what we need. Future Oncol, 2009. 5(3): p. 379-90.

29. Liu, X., et al., Zinc finger protein ZBTB20 promotes Toll-like receptor-triggered innate immune responses by repressing IkappaBalpha gene transcription. Proc Natl Acad Sci U S A, 2013. 110(27): p. 11097-102.

30. Zhou, S., et al., Role of interferon regulatory factor 7 in $T$ cell responses during acute lymphocytic choriomeningitis virus infection. J Virol, 2012. 86(20): p. 11254-65.

31. Suryawanshi, A., et al., Canonical wht signaling in dendritic cells regulates Th1/Th17 responses and suppresses autoimmune neuroinflammation. J Immunol, 2015. 194(7): p. 3295-304.

32. Uto, T., et al., Clec4A4 is a regulatory receptor for dendritic cells that impairs inflammation and T-cell immunity. Nat Commun, 2016. 7: p. 11273.

33. Chang, J., S.L. Kunkel, and C.H. Chang, Negative regulation of MyD88-dependent signaling by IL-10 in dendritic cells. Proc Natl Acad Sci U S A, 2009. 106(43): p. 1832732.

34. Hedrich, C.M., et al., Stat3 promotes IL-10 expression in lupus $T$ cells through transactivation and chromatin remodeling. Proc Natl Acad Sci U S A, 2014. 111(37): p. 1345762.

35. Garber, M., et al., A high-throughput chromatin immunoprecipitation approach reveals principles of dynamic gene regulation in mammals. Mol Cell, 2012. 47(5): p. 810-22.

36. Wu, L., A Flt3L encounter: mTOR signaling in dendritic cells. Immunity, 2010. 33(4): p. 580-2.

37. Thomson, A.W., H.R. Turnquist, and G. Raimondi, Immunoregulatory functions of mTOR inhibition. Nat Rev Immunol, 2009. 9(5): p. 324-37.

38. Raghav, S.K., et al., Integrative genomics identifies the corepressor SMRT as a gatekeeper of adipogenesis through the transcription factors C/EBPbeta and KAISO. Mol Cell, 2012. 46(3): p. 335-50.

39. Iyer, S.S. and G. Cheng, Role of interleukin 10 transcriptional regulation in inflammation and autoimmune disease. Crit Rev Immunol, 2012. 32(1): p. 23-63.

40. Liu, B.S., et al., TLR-mediated STAT3 and ERK activation controls IL-10 secretion by human B cells. Eur J Immunol, 2014. 44(7): p. 2121-9.

41. Samarasinghe, R., et al., Induction of an anti-inflammatory cytokine, $I L-10$, in dendritic cells after toll-like receptor signaling. J Interferon Cytokine Res, 2006. 26(12): p. 893-900. 
42. Brightbill, H.D., et al., A prominent role for Sp1 during lipopolysaccharide-mediated induction of the IL-10 promoter in macrophages. J Immunol, 2000. 164(4): p. 1940-51.

43. Haidinger, M., et al., A versatile role of mammalian target of rapamycin in human dendritic cell function and differentiation. J Immunol, 2010. 185(7): p. 3919-31.

44. Chen, Z., et al., Selective regulatory function of Socs3 in the formation of IL-17-secreting T cells. Proc Natl Acad Sci U S A, 2006. 103(21): p. 8137-42.

45. Rigby, R.J., et al., Suppressor of cytokine signaling 3 (SOCS3) limits damage-induced crypt hyper-proliferation and inflammation-associated tumorigenesis in the colon. Oncogene, 2007. 26(33): p. 4833-41.

46. Lee, S.O., et al., The nuclear receptor TR3 regulates mTORC1 signaling in lung cancer cells expressing wild-type p53. Oncogene, 2012. 31(27): p. 3265-76.

47. Heming, M., et al., Peroxisome Proliferator-Activated Receptor-gamma Modulates the Response of Macrophages to Lipopolysaccharide and Glucocorticoids. Front Immunol, 2018. 9: p. 893.

48. Ohta, A., et al., Manipulation of Th1/Th2 balance in vivo by adoptive transfer of antigenspecific Th1 or Th2 cells. J Immunol Methods, 1997. 209(1): p. 85-92.

49. $\mathrm{Li}$, S., et al., Effector $\mathrm{T}$ helper cell populations are elevated in the bone marrow of rheumatoid arthritis patients and correlate with disease severity. Sci Rep, 2017. 7(1): p. 4776.

50. Smita, S., et al., Importance of EMT Factor ZEB1 in cDC1 "MutuDC Line" Mediated Induction of Th1 Immune Response. Front Immunol, 2018. 9: p. 2604.

51. Smita, S., et al., Zbtb10 transcription factor is crucial for murine cDC1 activation and cytokine secretion. Eur J Immunol, 2021.

52. Alignani, D., et al., Orally administered OVA/CpG-ODN induces specific mucosal and systemic immune response in young and aged mice. J Leukoc Biol, 2005. 77(6): p. 898-905.

53. Rainone, V., et al., Immunological Characterization of Whole Tumour Lysate-Loaded Dendritic Cells for Cancer Immunotherapy. PLoS One, 2016. 11(1): p. e0146622.

54. Trivedi, U.H., et al., Quality control of next-generation sequencing data without a reference. Front Genet, 2014. 5: p. 111.

55. Kim, D., et al., Graph-based genome alignment and genotyping with HISAT2 and HISATgenotype. Nat Biotechnol, 2019. 37(8): p. 907-915.

56. Liao, Y., G.K. Smyth, and W. Shi, featureCounts: an efficient general purpose program for assigning sequence reads to genomic features. Bioinformatics, 2014. 30(7): p. 923-30.

57. Love, M.I., W. Huber, and S. Anders, Moderated estimation of fold change and dispersion for RNA-seq data with DESeq2. Genome Biol, 2014. 15(12): p. 550.

58. H, W., ggplot2: Elegant Graphics for Data Analysis. 2016.

59. Langmead, B. and S.L. Salzberg, Fast gapped-read alignment with Bowtie 2. Nat Methods, 2012. 9(4): p. 357-9.

60. Li, H., et al., The Sequence Alignment/Map format and SAMtools. Bioinformatics, 2009. 25(16): p. 2078-9.

61. Picard toolkit. Broad Institute, GitHub repository, 2019.

62. Yu, G., L.G. Wang, and Q.Y. He, ChIPseeker: an R/Bioconductor package for ChIP peak annotation, comparison and visualization. Bioinformatics, 2015. 31(14): p. 2382-3.

63. Neph, S., et al., BEDOPS: high-performance genomic feature operations. Bioinformatics, 2012. 28(14): p. 1919-20.

64. Shen L, S.I., GeneOverlap: Test and visualize gene overlaps. 2020.(R package version 1.26.0,).

65. Heinz, S., et al., Simple combinations of lineage-determining transcription factors prime cis-regulatory elements required for macrophage and B cell identities. Mol Cell, 2010. 38(4): p. 576-89. 
66. Yu, G., et al., clusterProfiler: an R package for comparing biological themes among gene clusters. OMICS, 2012. 16(5): p. 284-7.

\section{Figure legends:}

\section{Figure 1. SMRT depleted $\mathrm{CDC1}$ and BMcDC1 exhibit enhanced activation and maturation upon TLR9 ligation with CpG-B}

A. RT-qPCR showing relative transcript expression of smrt, Il-12b and Il-10 in unstimulated, 2h and 6h CpG-B stimulated control and SMRT KD cDC1. (n=3)

B. Western blot showing SMRT protein expression in unstimulated and $6 \mathrm{~h} \mathrm{CpG}$ stimulated control and SMRT KD cDC1. Densitometric analysis depicted normalized intensity of SMRT bands in SMRT KD cDC1 and control cells. Housekeeping gene $\beta$-actin was used as loading control. ( $\mathrm{n}=3)$.

C. Flow cytometry analysis of co-stimulatory surface markers CD80, CD86, MHC-II, MHC-I, and CD40 in unstimulated and 6h CpG stimulated control and SMRT KD cDC1 mutuDCs. Corresponding contour plots, dot plots, and bar-plot showed the percent positive cells and MFI shifts for each of the marker gene. $(n=5-6)$

D. Flow cytometry analysis depicting MFI histograms of co-stimulatory molecules CD80 and CD86 in 6h CpG stimulated control and SMRT KD bone-marrow derived cDC1 (BMcDC1). $(\mathrm{n}=9)$

$* \mathrm{p} \leq 0.05, * * \mathrm{p} \leq 0.01$ and ${ }^{* * *} \mathrm{p} \leq 0.001$. $\mathrm{p}$-value has been calculated using two tailed paired student's t-test. Data shown in figure is combined from 3 independent experiments [A and B] and from 4 independent experiments [C-D]. Error bars represent SEM.

\section{Figure 2. Activated SMRT KD cDC1 showed enhanced inflammatory cytokine expression.}

A. Flow cytometry analysis depicting the intracellular cytokine expression of IL-6, IL12p40, IL-23p19, and IL-10 cytokine expression in control and SMRT KD cDC1 before and after 6h $\mathrm{CpG}$ challenge. Corresponding contour plot, dot plot and bar-plots shows the percent positive cell population and MFI shifts respectively. ( $\mathrm{n}=6)$

B. Bioplex cytokine assay showing the estimation of secreted cytokines IL-6, IL-12p40, IL-12p70, IL-10, and IL-2 in the culture supernatants of control and SMRT KD cDC1 before and after 6h CpG activation. $(n=4-5)$ 
C. Flow cytometry analysis depicting the MFI histogram and scatter dot plots depicting the flow cytometry analysis for intra-cellular expression of pro-inflammatory cytokines IL6, IL-23p19 and anti-inflammatory cytokine IL-10 in 6h CpG stimulated control and SMRT KD bone-marrow derived cDC1 (BMcDC1). (n=3-10)

D. Bioplex cytokine assay showing the quantification of IL-6, IL-12p40, IL-12p70, and IL2 cytokine secreted in the culture supernatant of $6 \mathrm{~h}$ CpG stimulated control and SMRT KD BMcDC1s. (n=5)

${ }^{*} \mathrm{p} \leq 0.05,{ }^{* *} \mathrm{p} \leq 0.01$ and ${ }^{* * *} \mathrm{p} \leq 0.001$. $\mathrm{p}$-value has been calculated using two tailed paired student's t-test. Data shown in figure is combined from 4 independent experiments [A], 2-3 independent replicates [B], 3-4 independent replicates [C], and from 2 independent experiments [D]. Error bars represent SEM.

\section{Figure 3. SMRT KD cDC1s enhanced Th1 and Th17 cell polarization ex vivo.}

A. Flow cytometry analysis depicting dot-plot showing the proliferating populations of OT-II Th-cells co-cultured with control and SMRT KD cDC1s pulsed with OVA 323339 peptide overnight followed by CpG challenge.

B. Flow cytometry analysis showing bar-plot demonstrating the percentage of parent or P1 population of OT-II Th-cells co-cultured with control and SMRT KD cDC1s pulsed with OVA 323-339 peptide overnight followed by CpG challenge. ( $n=5$ )

C. Flow cytometry analysis showing bar-plot demonstrating the percentage of proliferating or P2 population of OT-II Th-cells co-cultured with control and SMRT KD cDC1s pulsed with OVA 323-339 peptide overnight followed by $\mathrm{CpG}$ challenge. $(\mathrm{n}=5)$

D. Flow cytometry analysis showing contour and scatter dot plots representing co-cultured OT-II T-cells showing signature transcription factor and cytokine for Th1, T-bet and IFN- $\gamma$ response in unstimulated and $\mathrm{CpG}$ stimulated condition. $(\mathrm{n}=10)$

E. Flow cytometry analysis showing contour and dot plots showing ROR $\gamma$ t and IL17A as signature transcription factor and cytokines for Th17 subtype generated from cocultured OT-II T-cells in unstimulated and CpG stimulated condition. $(\mathrm{n}=5)$ 
F. Bioplex cytokine assay showing quantification of secretory cytokine IL-17 cytokine from supernatant of $\mathrm{CD}^{+}{ }^{+}$T-cells co-cultured with CpG stimulated control and SMRT KD DCs. $(n=5)$

G. RT-qPCR showing relative mRNA expression of Smrt transcript (1/dCT) in RA patients $(n=11)$ and their healthy counterparts. $(n=14)$

${ }^{*} \mathrm{p} \leq 0.05, * * \mathrm{p} \leq 0.01$ and $* * * \mathrm{p} \leq 0.001$. $\mathrm{p}$-value has been calculated using two tailed unpaired student's t-test. Data shown in figure is combined from 3 independent experiments [A-C], 4 independent replicates [D-E], and 3 independent experiments [F]. Error bars represent SEM.

\section{Figure 4. SMRT KD cDC1s increased perforin, granzyme, and IFN- $\gamma$ production $e x$ vivo in CD8 T lymphocytes}

A. Flow cytometry analysis showing dot plot of the proliferation of co-cultured OT-I Tcells co-cultured with control and SMRT KD cDC1s pulsed with OVA 257-264 peptide overnight followed by CpG challenge.

B. Flow cytometry analysis showing the bar-plot demonstrating the percentage of dividing or P1 and proliferating or P2 population of OT-I T-cells co-cultured with control and SMRT KD cDC1s pulsed with OVA peptide overnight followed by CpG challenge. $(n=4)$

C. Flow cytometry analysis showing the contour plot and scatter dot plots representing cocultured OT-I T-cells showing cytolytic IFN- $\gamma, \mathrm{GrB}$, and Perforin in control and SMRT KD DCs in unstimulated and CpG stimulated condition. ( $\mathrm{n}=9)$

${ }^{*} \mathrm{p} \leq 0.05, * * \mathrm{p} \leq 0.01$ and ${ }^{* * *} \mathrm{p} \leq 0.001$. $\mathrm{p}$-value has been calculated using two tailed unpaired student's t-test. Data shown in figure is combined from 2 independent experiments [A \& B], 2-4 independent replicates [C]. Error bars represent SEM.

Figure 5. Induction of DTH response and tumor regression in C57BL/6 after adoptive transfer of Control and SMRT KD DCs

A. Experimental design depicting DTH model to understand the effect of SMRT KD in comparison to control cells through ova immunisation, sensitisation and rechallenge followed by measurement of paw thickness. 
B. Line plot with standard error mean showing footpad swelling till $72 \mathrm{~h}$ post antigen rechallenge. Footpad swelling was calculated by subtracting the paw thickness (mm) in the right footpad (pbs injection) from left footpad (ova injection). (n=4-6)

C. Flow cytometry analysis showing scatter dot and contour plot representing Th1 subtype marked by enhanced IFN- $\gamma$ and T-bet in SMRT KD DCs isolated from popliteal lymph nodes $72 \mathrm{~h}$ post antigen/ova rechallenge. $(\mathrm{n}=4-6)$

D. Flow cytometry analysis depicting scatter dot and contour plots representing Th17 subtype marked by enhanced IL-17 and ROR $\gamma \mathrm{t}$ in SMRT KD DCs isolated from popliteal lymph nodes $72 \mathrm{~h}$ post antigen/ova rechallenge. $(\mathrm{n}=4-6)$

E. Experimental design of melanoma model to understand the effect of SMRT KD DCs on tumor regression. Mice were first given tumor vaccination 10 and 7 days prior to tumor injection using DCs pulsed with B16F10 lysates.

F. Line plot with standard error mean showing tumor volume that was taken every day starting from 7th day after B16F10 injection till 16 days post tumor rechallenge in PBS, control and SMRT KD DCs injected mice. Tumor volume was calculated as tumor volume $=\left(\right.$ tumor length $\mathrm{x}$ tumor width $\left.{ }^{2}\right) / 2 .(\mathrm{n}=4-6)$

G. Images showing tumor size isolated from mice at 16 days post B16F10 rechallenge in PBS, control and SMRT KD DCs injected mice. $(\mathrm{n}=4)$

H. Flow cytometry analysis showing dot plots representing cytotoxic cytokine such as perforin, GrB, IFN- $\gamma$ from single cell suspension of tumours that were isolated 16 days post B16F10 rechallenge in PBS, control and SMRT KD DCs injected mice. $(\mathrm{n}=6)$

I. Flow cytometry analysis showing dot plots showing tumor weight isolated 16 days post B16F10 rechallenge in PBS, control and SMRT KD DCs injected mice. $(\mathrm{n}=6)$

${ }^{*} \mathrm{p} \leq 0.05,{ }^{*} \mathrm{p} \leq 0.01$ and ${ }^{* * *} \mathrm{p} \leq 0.001 . \mathrm{p}$-value has been calculated using two tailed unpaired student's t-test. Data shown in figure is combined from 2 independent experiments [A-I]. Error bars represent SEM. 
Figure 6. Integrative genomics analysis (RNA-seq and ChIP-seq) identified the differential role of SMRT and NCoR1 in regulation of immune response in cDC1.

A. Volcano plot showing the differentially expressed genes (DEGs) in SMRT KD cDC1 DCs as compared to control cells after 6h CpG stimulation. There were 957 and 813 genes upregulated and downregulated respectively upon SMRT depletion. $(n=2)$

B. Bar plot showing the total number of SMRT bound and unbound DEGs in unstimulated and 6h CpG stimulated SMRT KD cDC1 DCs. p-value showing significance of overlap between SMRT bound genes and DEGs.

C. Bar plot depicting enriched canonical pathways for the list of SMRT bound upregulated DEGs in 6h CpG activated SMRT KD cDC1 DCs as compared to control cells using Ingenuity pathway analysis (IPA).

D. Bar plot depicting enriched canonical pathways for the list of significantly SMRT bound down-regulated DEGs in 6h CpG activated SMRT KD cDC1 DCs as compared to control cells using Ingenuity pathway analysis (IPA).

E. Bar plot showing comparison of number of total DEGs after SMRT KD and NCoR1 $\mathrm{KD}$ in unstimulated and $6 \mathrm{~h}$ CpG stimulation condition.

F. Heatmap showing two clusters obtained from K-means clustering of log2 fold change of DEGs upon SMRT and NCoR1 KD compared to control cells in unstimulated and 6h CpG stimulation condition ( $1^{\text {st }}$ panel). The box plot shows scaled normalized expression values (rlog) from DESeq2 for the respective cluster ( $2^{\text {nd }}$ panel).

G. Bar plot showing enriched canonical pathways from IPA for respective clusters shown in Fig 6F.

H. Bar plot with standard deviation showing normalized count from DESeq2 of selected genes from enriched pathway for cluster-1 (Il-12b and Il-6) and cluster2 (Il-10 and Socs3).

I. Tornado plot showing ChIP-seq signal ( $\pm 2 \mathrm{~kb}$ to peak center) of differential NCoR 1 and SMRT binding sites (NCoR1 CpG and SMRT CpG) in unstimulated and 6h CpG stimulation ( $1^{\text {st }}$ panel). Bar plot showing the distribution of differential genomic regions based on distance relative to TSS ( $2^{\text {nd }}$ panel).

J. Box plot showing normalized tag count in differential NCoR1 CpG and SMRT CpG cluster. Boxes encompass the $25^{\text {th }}$ to $75^{\text {th }}$ percentile of normalized tag count. Whiskers 
extend to the $10^{\text {th }}$ and $90^{\text {th }}$ percentiles. Mean difference significance was calculated using the Wilcoxon test.

K. Table showing transcription factor motifs that were significantly enriched (P-value < 1e-10) in differential NCoR1 CpG and SMRT CpG clusters.

L. Dot plot showing the significantly enriched KEGG terms for genes associated with NCoR1 and SMRT CpG binding clusters shown in Fig 6I. KEGG term enrichment analysis was performed using cluterProfiler $\mathrm{R}$ package.

\section{Figure 7. Nurr-77, mTOR, Stat3 signaling regulates IL-10 expression in SMRT KD cDCs.}

A. Illustration of Jak/Stat Signaling pathway from Ingenuity pathway analysis with mapped expression of genes. (Red and green indicates high and low expression respectively).

B. RNA-seq analysis showing heatmap showing Log2 (fold change) of all the genes in KEGG Jak-Stat signaling term shown in Fig 6L.

C. Western blot depicting phospho-STAT3, total STAT3, and $\beta$-actin protein levels in unstimulated and 2h and 6h CpG stimulated control, NCoR1 KD, and SMRT KD cDC1s.

D. Bar plot with standard error mean from densitometric analysis depicting normalized intensity of phosphorylated STAT3 bands in control and KD cDC1s. Housekeeping gene $\beta$-actin was used as loading control. $(n=3)$.

E. Bar plot with standard error mean showing \% input enrichment from ChIP-qPCR of phospho-STAT3 on IL-10 enhancer region in 2h CpG stimulated control, NCoR1 KD, and SMRT KD cDC1s. $(\mathrm{n}=3)$

F. Western blot depicting the levels of phosphorylated mTOR, total mTOR, and GAPDH in unstimulated, 2h, and 6h CpG stimulated control, NCoR1 KD, and SMRT KD cDC1s. Housekeeping gene GAPDH was used as loading control.

G. Bar plot with standard error mean from densitometric analysis depicting normalized intensity of phosphorylated mTOR bands in control and KD cDC1s. Housekeeping gene GAPDH was used as loading control. $(n=3)$ 
H. Bar plot with standard deviation showing normalized count of Nr4a1 gene in NCoR1 and SMRT KD cDC1 with their respective matched controls at $0 \mathrm{~h}$ and $6 \mathrm{~h} \mathrm{CpG}$ stimulation.

I. Western blot depicting the levels of NR4A1 and $\beta$-actin in unstimulated, $2 \mathrm{~h}$, and $6 \mathrm{~h}$ CpG stimulated control and SMRT KD cDC1s. $(\mathrm{n}=3)$

J. Bar plot with standard error mean from densitometric analysis depicting the normalized intensity of NR4A1 bands in KD and control cells. Housekeeping gene $\beta$-actin was used as loading control. $(\mathrm{n}=3)$.

K. Western blot depicting the levels of phospho-mTOR, total mTOR, phospho-STAT3, total STAT3, NR4A1, and $\beta$-actin in 2h CpG stimulated control and NR4A1 KD cDC1s.

L. Bar plot with standard error mean from densitometric analysis depicting the normalized intensity of phosphorylated-mTOR, phosphorylated-STAT3, and NR4A1 in 2h CpG stimulated control and NR4A1 KD cDC1s. Housekeeping gene $\beta$-actin was used as loading control. $(n=3)$

M. Percent positive cells and MFI depicting flow cytometry analysis of the antiinflammatory cytokine IL-10 in 6h CpG stimulated control and NR4A1 KD DCs. (n=6)

N. Relative transcript expression of Smrt, Nr4a1, Il-10, and Il-12b transcript in 6h CpGB stimulated SMRT KD, CpG along with vehicle treated SMRT KD and CpG along with 6-MP treated SMRT KD DCs as estimated by RT-qPCR. (n=3)

$* \mathrm{p} \leq 0.05, * * \mathrm{p} \leq 0.01$ and $* * * \mathrm{p} \leq 0.001$. $\mathrm{p}$-value has been calculated using two tailed paired student's t-test. Data shown in figure is combined from 3 independent experiments [C-L], 4 independent experiments [M], and 3 independent replicates [N]. Error bars represent SEM.

\section{Supplementary table legends}

Table S4:

Excel file having list of differentially expressed genes in SMRT KD compared to control at $0 \mathrm{~h}$ and $6 \mathrm{~h} \mathrm{CpG}$ stimulation.

\section{Table S5:}


Excel file having list of annotation of direct target genes that are bound and differentially expressed upon SMRT KD at Oh and 6h CpG stimulation.

\section{Table S6:}

Excel file having list of enriched Ingenuity pathway terms for direct target genes of SMRT that upregulated and downregulated in SMRT KD compared to control at $6 \mathrm{~h} \mathrm{CpG}$ stimulation.

\section{Table S7:}

Excel file having total list of DEGs in any of the comparison (NCoR1 KD and SMRT KD) divided in $6 \mathrm{k}$-means clusters.

\section{Table S8:}

Excel file having list of enriched IPA pathway terms for all the $6 \mathrm{k}$-means clusters.

Table S9:

Excel file having list of enriched KEGG terms for list of direct target genes of differential binding sites of NCoR1 and SMRT. 
Figure 1.

(A)

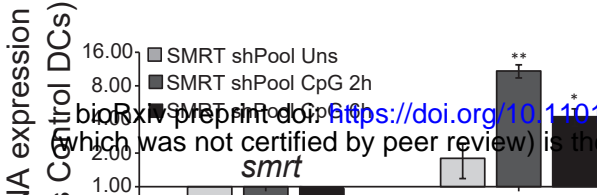

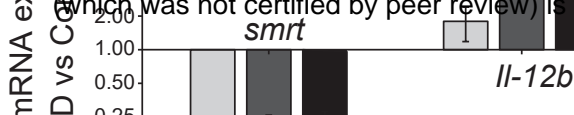

E

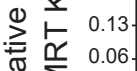

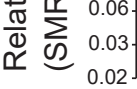

(B)

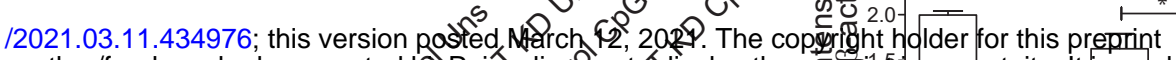

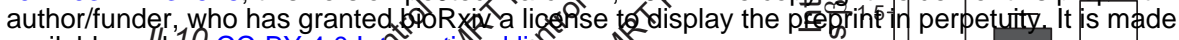

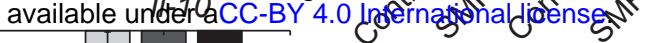
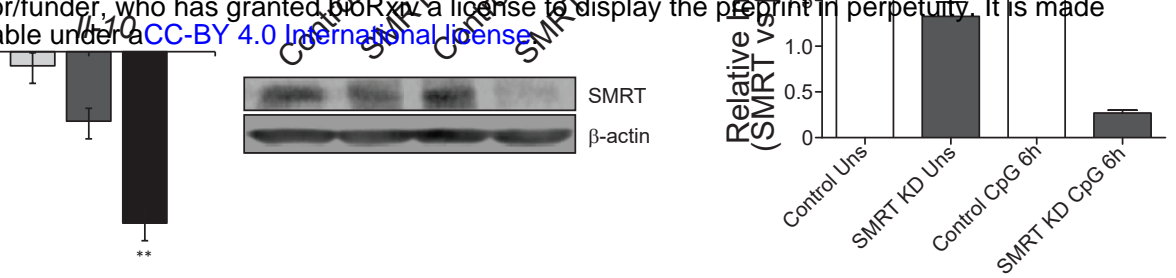

(C)
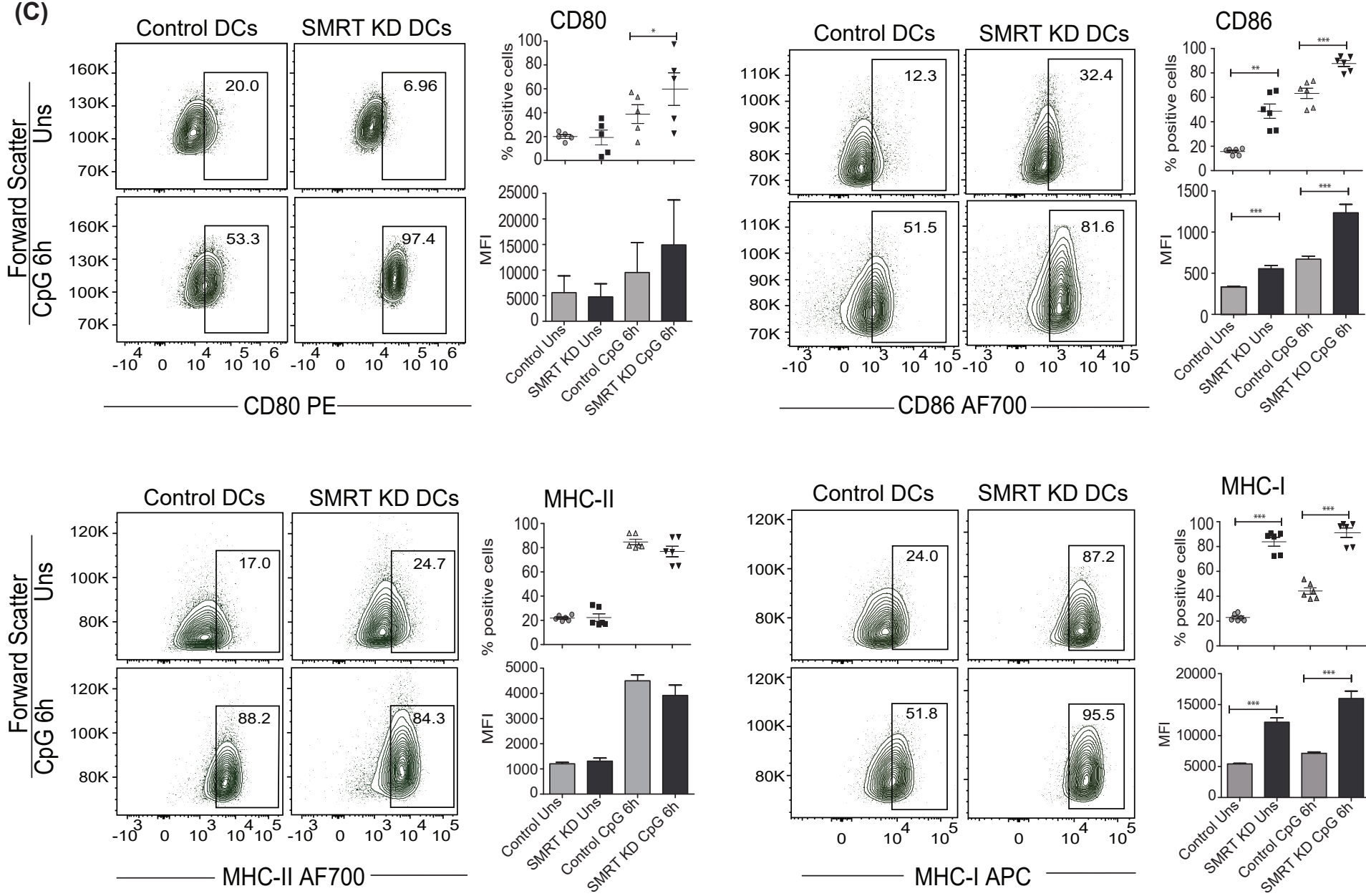

MHC-I
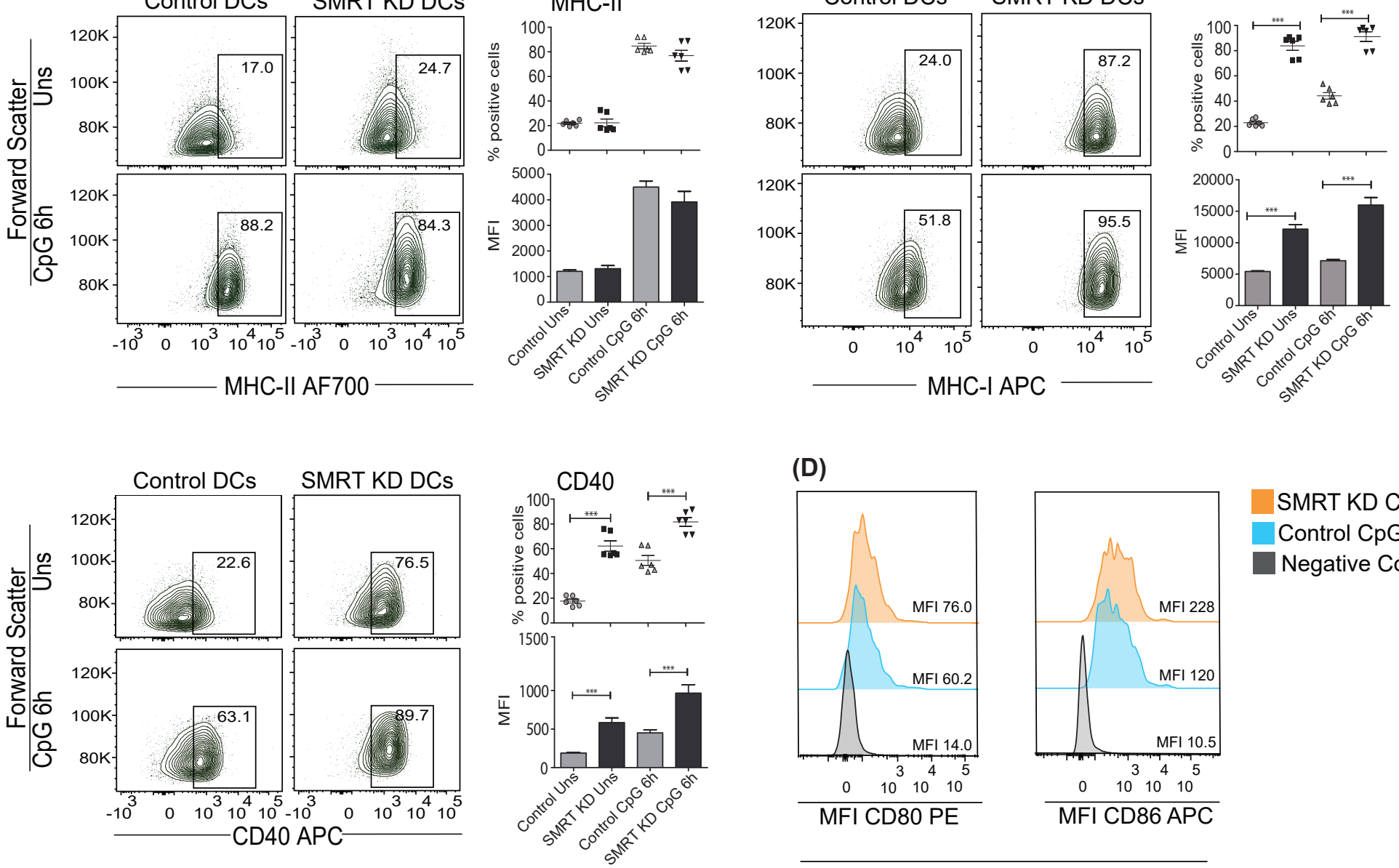

(D)

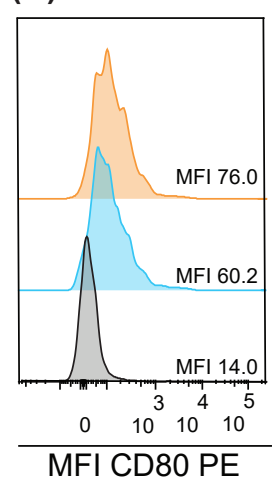

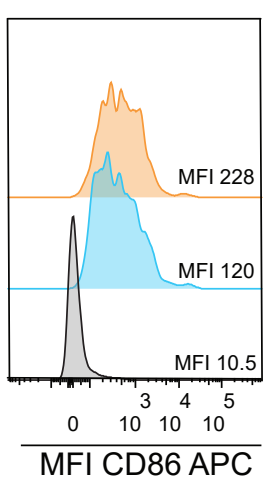

SMRT KD CpG

Control $\mathrm{CpG}$

Negative Control

BMcDC1 CpG $6 \mathrm{~h}$ 
Figure 3
(A)
Control DCs
SMRT KD DCs
(B)
SMRTKD DCs

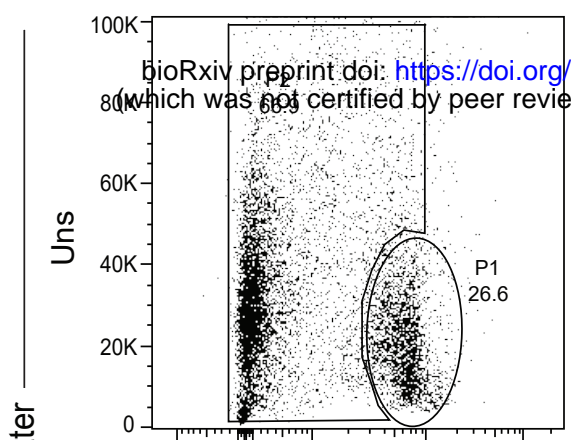

101101

$\left.\check{0}^{50}\right] \mathrm{T}$

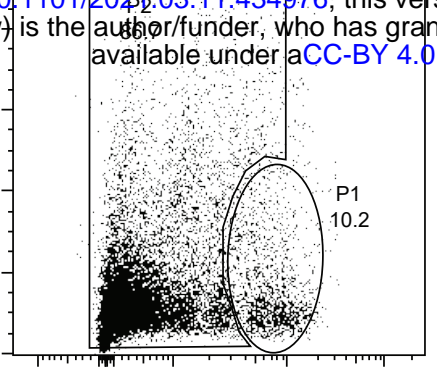

壱
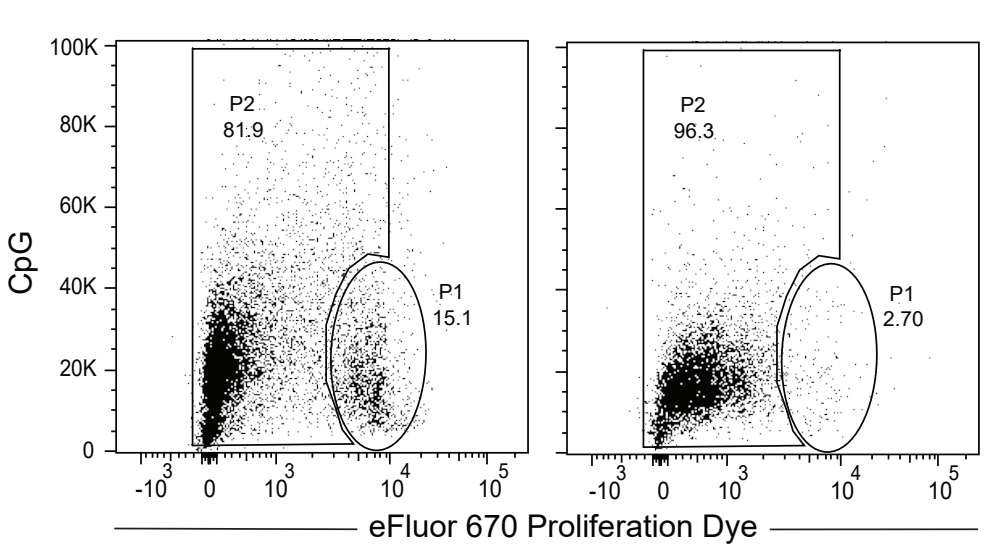

(C)

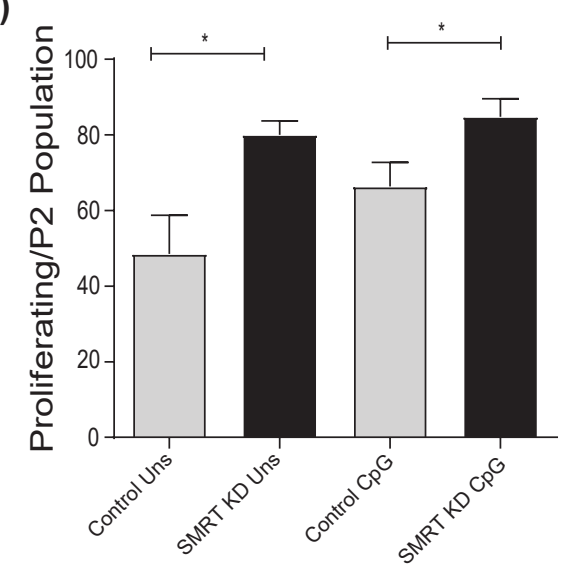

(D)

eFluor 670 Proliferation Dye
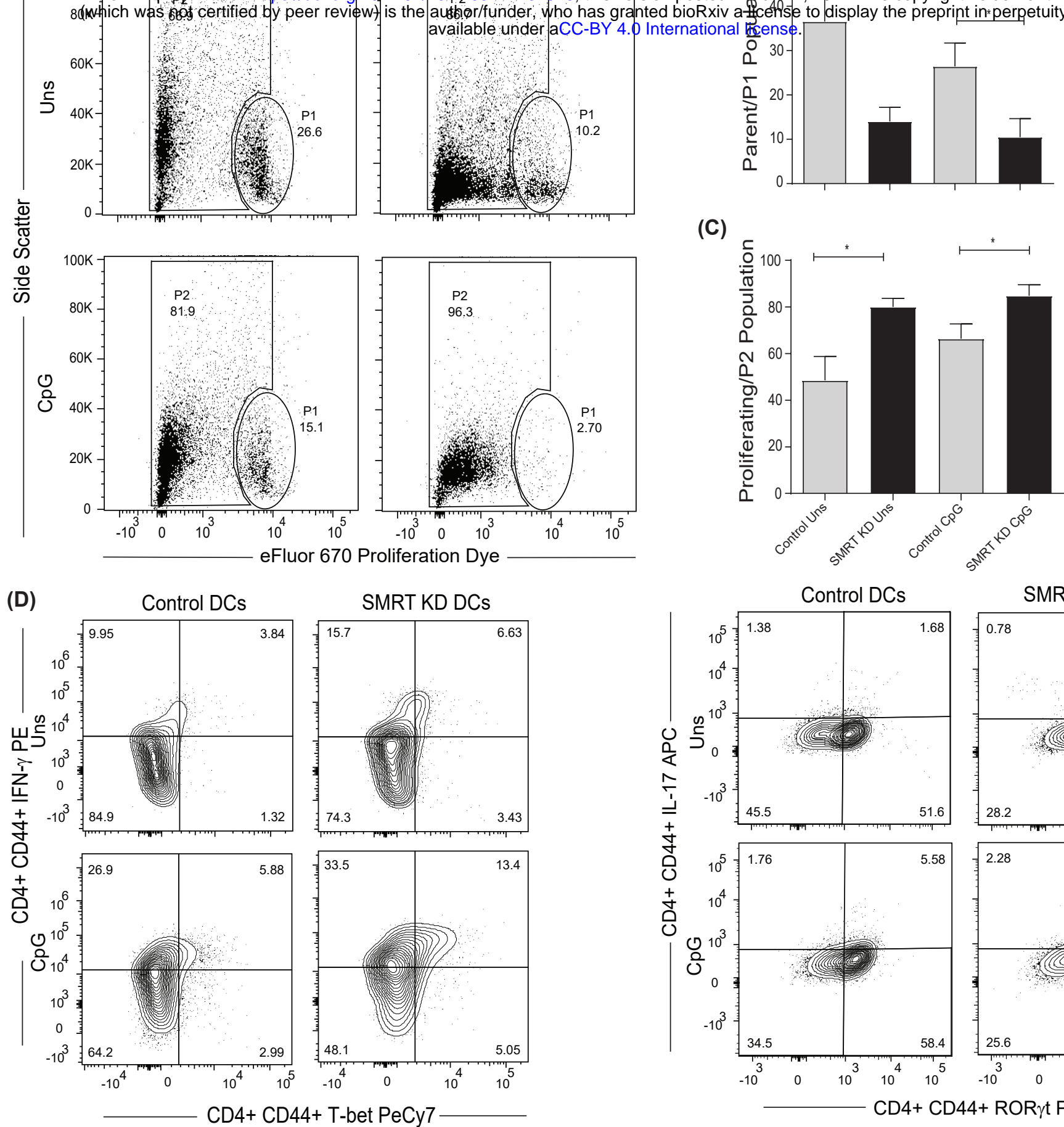

SMRT KD DCs

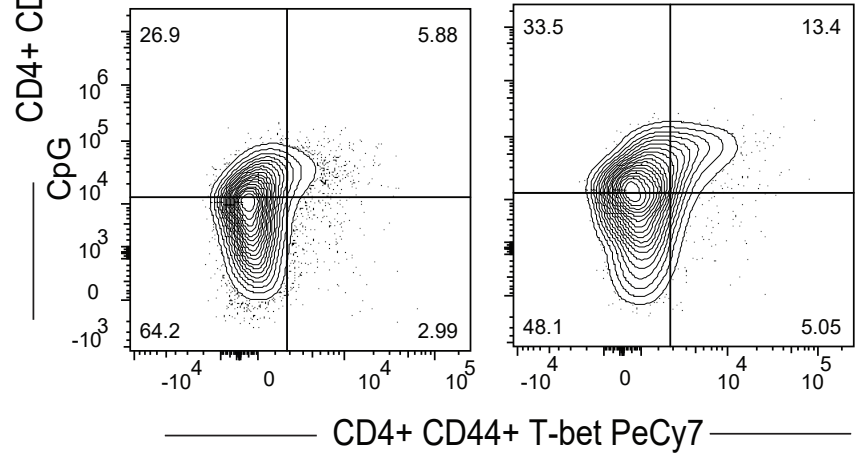

(F)

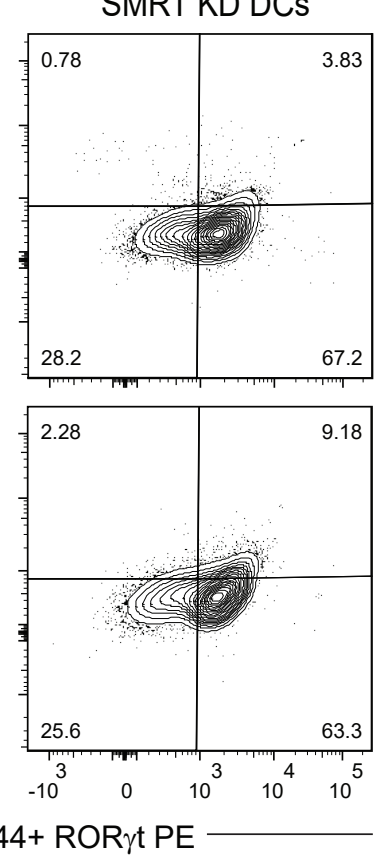

(G)
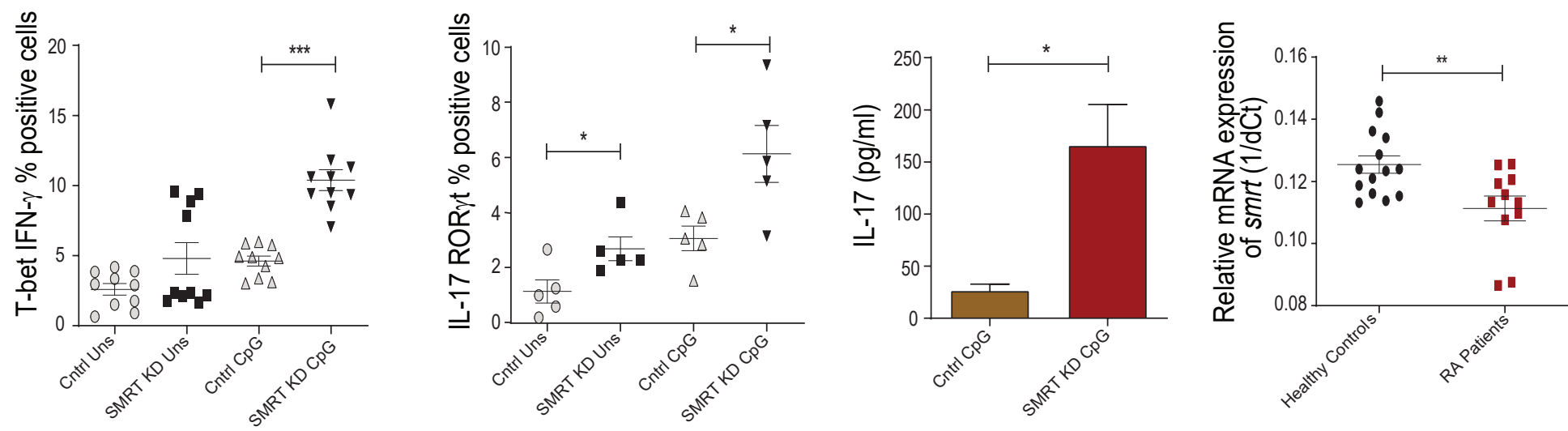
Figure 4

(A)

(B)

bioRxiv preprint doi: hitps: //doi.org/10.1101/2021.03.11.4349-76; this version posted March ${ }^{8} 12,2021$. The copyright holder for this preprint 250/wwich was not certified by peer review is the author/funder, who has granted bioRxiv a liense to display the preprint in perpetuity. It is made
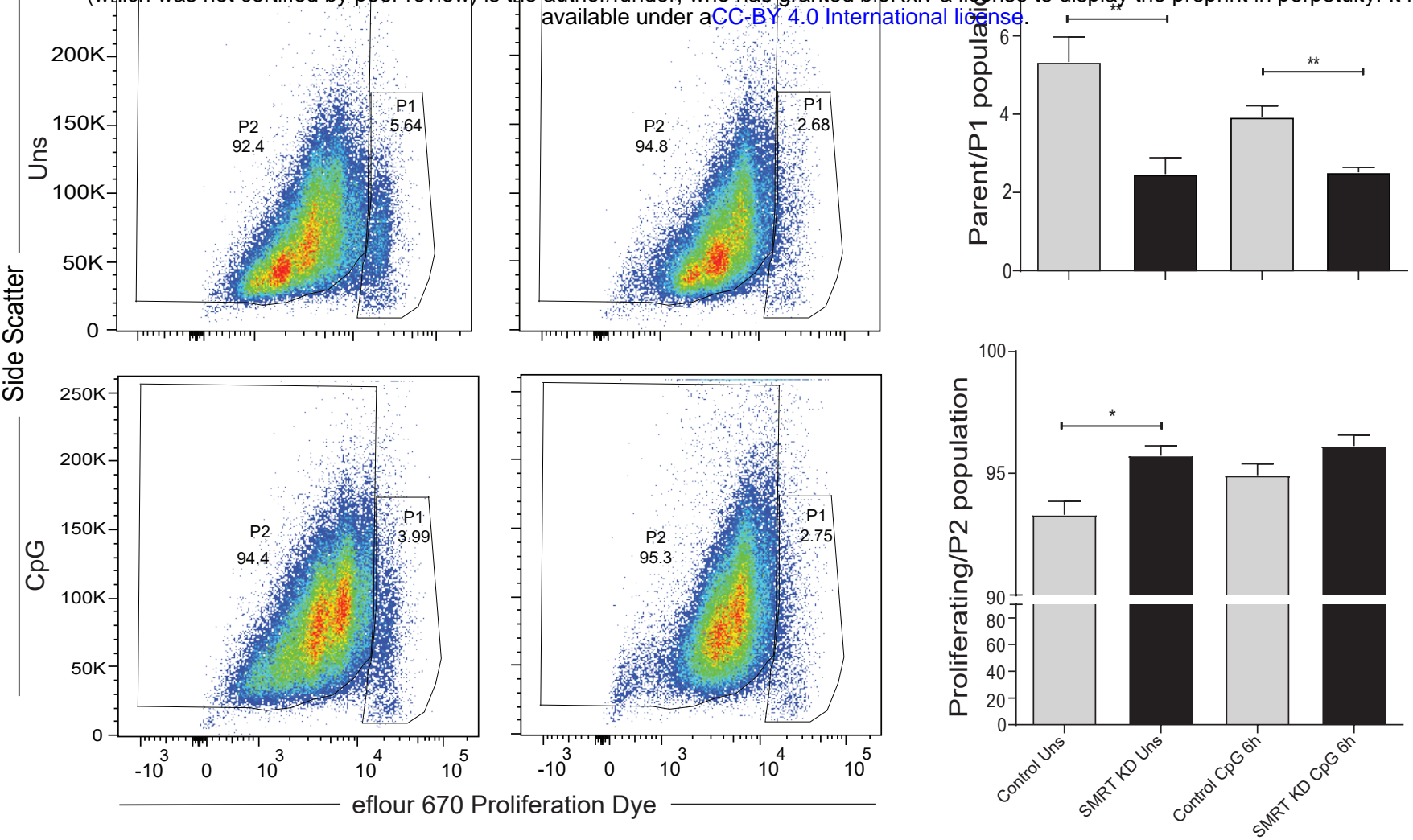

(C)
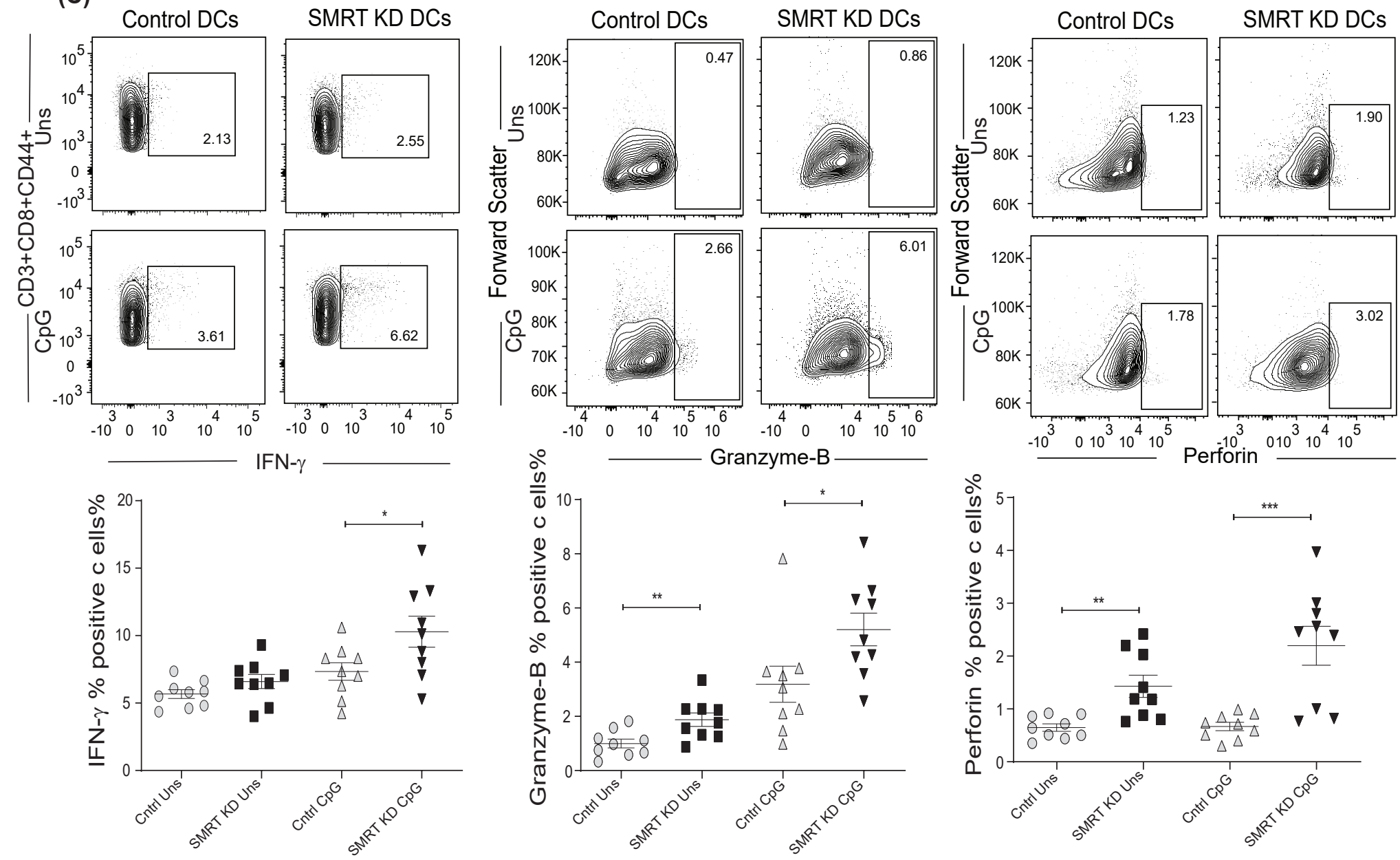
(A)

bioRxiv preprint doi: https://doi.org/10.1101/2021.03.11.434976; this version posted March 12, 2021. The copyright holder for this preprint

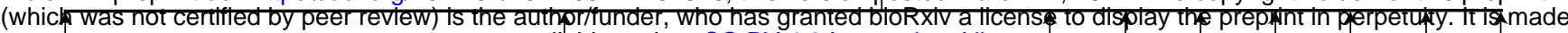

Day 0

OVA Immunisation

(subcutaneous)

(OVA+alum) available under aCC-BY 4.0 International license.

Day 14

DC Adoptive Transfer (intra-peritoneal)
Oh 12h 24h 36h 48h 60h 72h Paw thickness measurements (in hours)
(B)

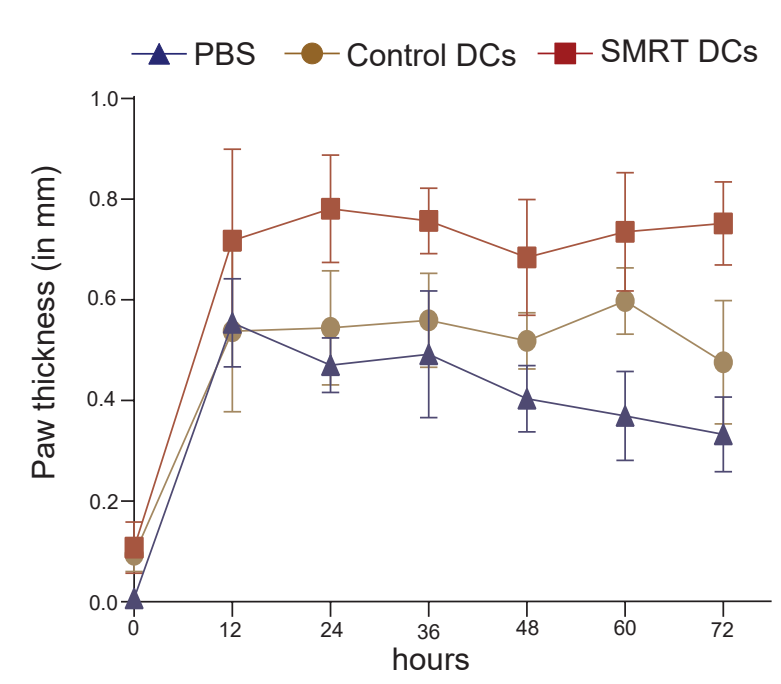

(C)
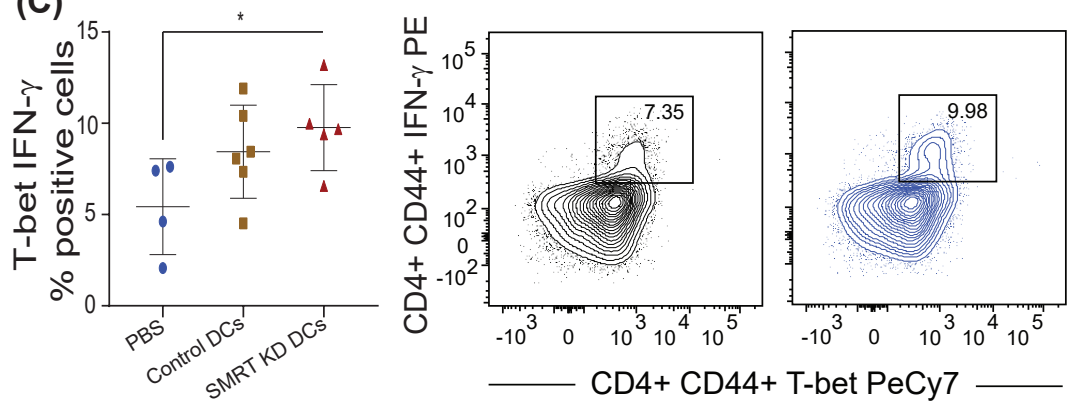

(D)
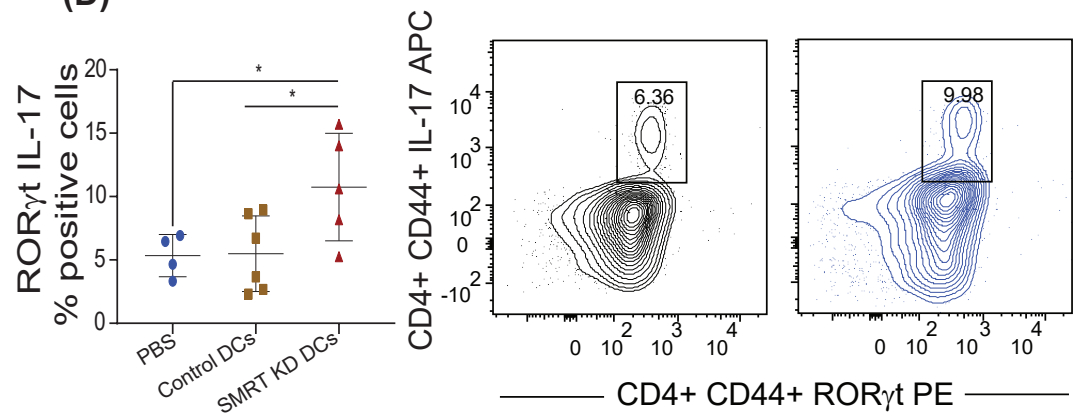

- CD4+ CD44+ T-bet PeCy7

(E)

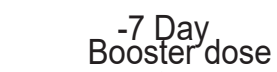

-10 Day

DC pulsed with B16F10 lysate (subcutaneously injected in flank)

(F)

(subcutaneously injected
in opposite flank)

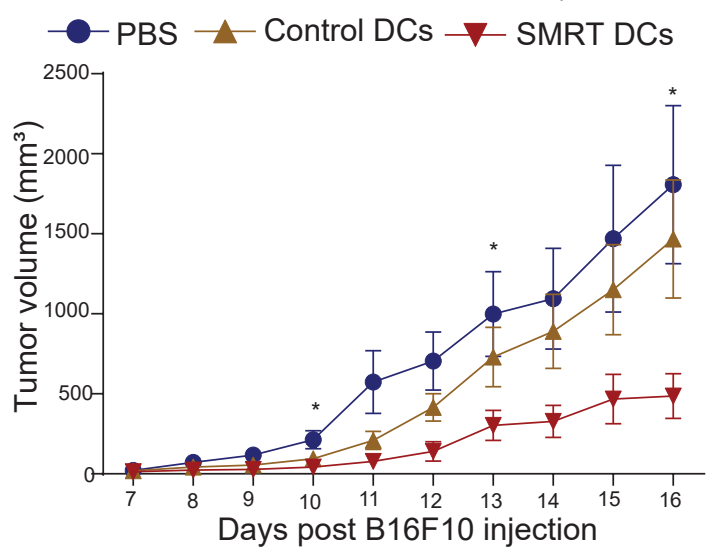

(H)
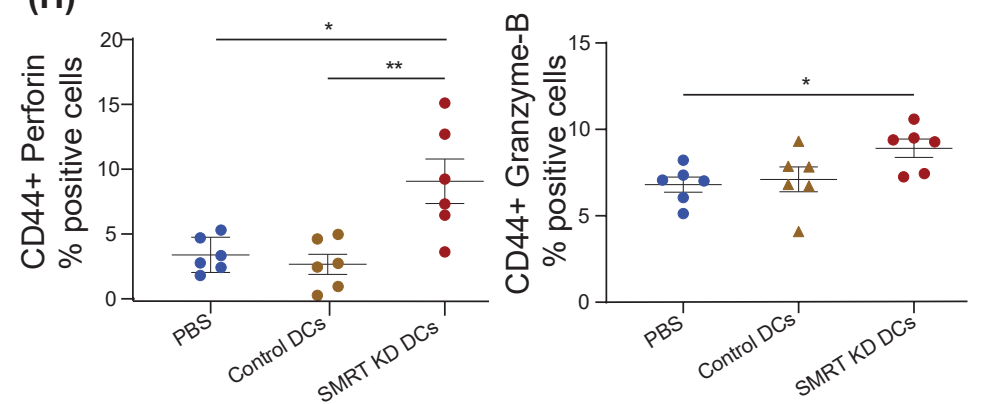

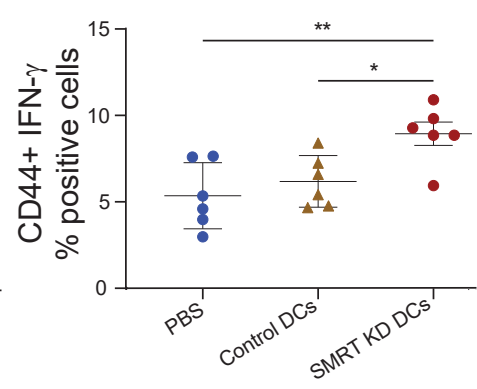

(G)

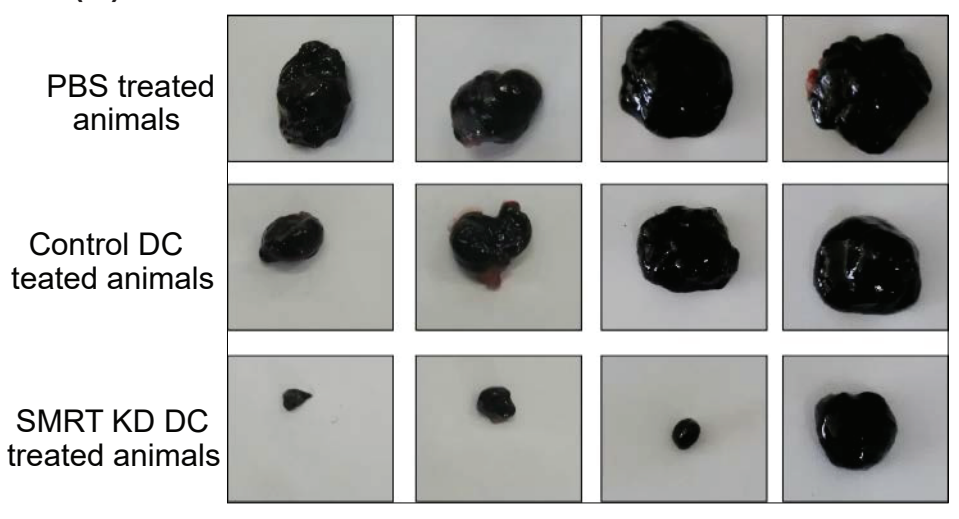

(I)

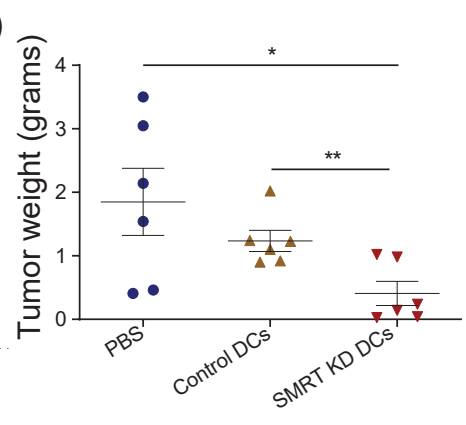


Figure 6

(A)

(B)

(C)

SMRT Unbound Up-regulated pathways
Maturation
Matic Cell

(E)

Up Down

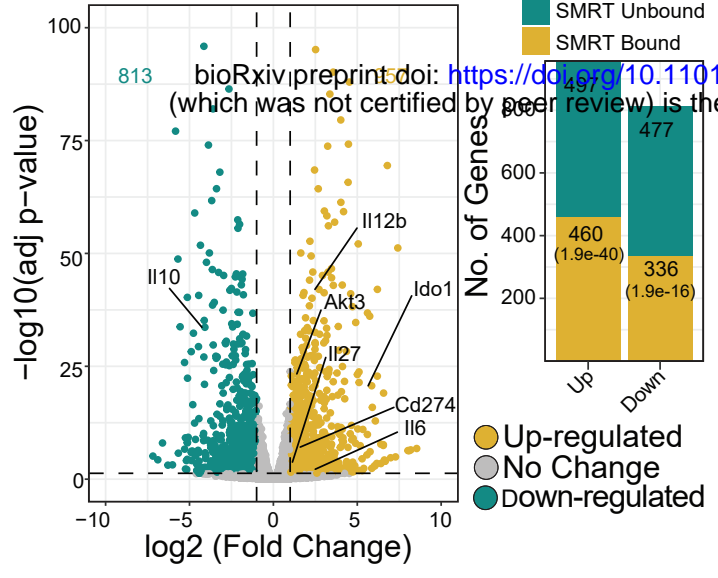

(D)

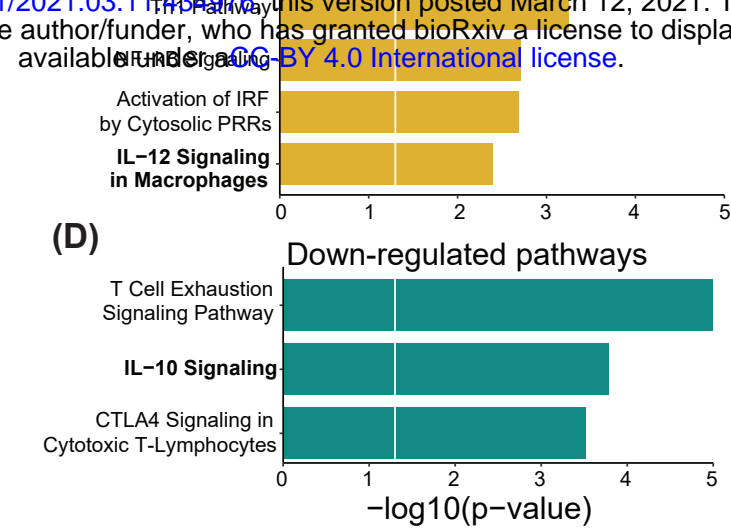

(G)

(F)

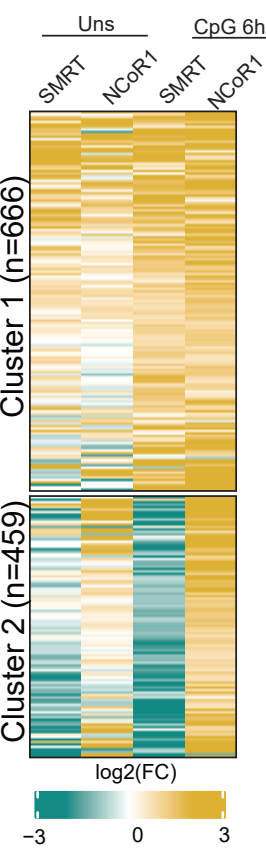

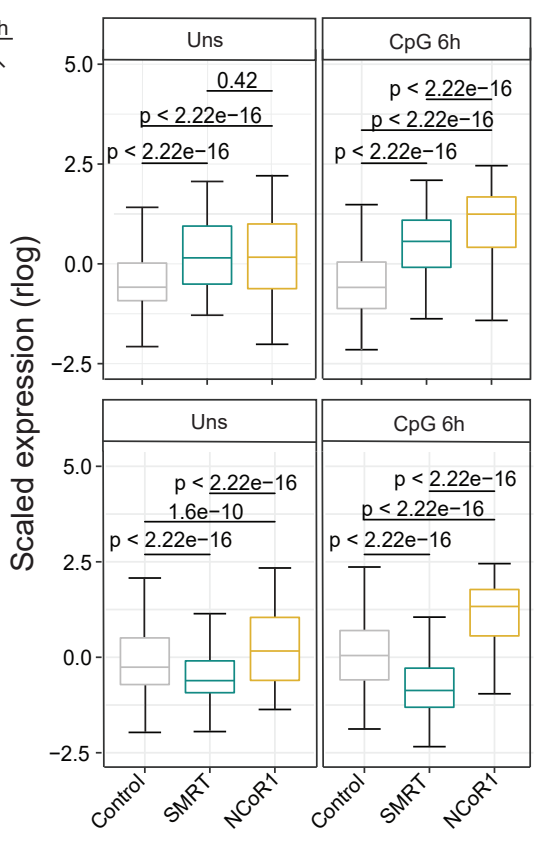

\section{(H)}

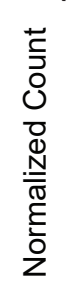

(I)

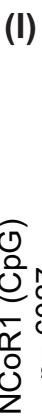

(J)

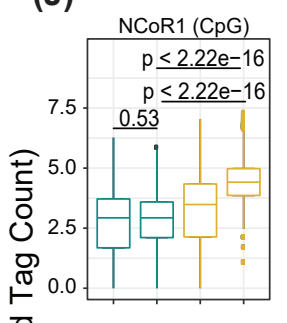

(K)

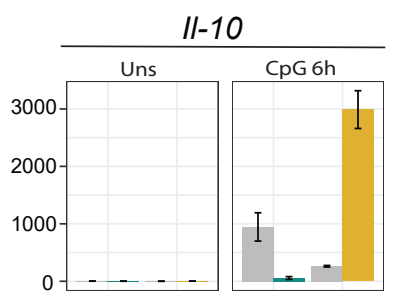

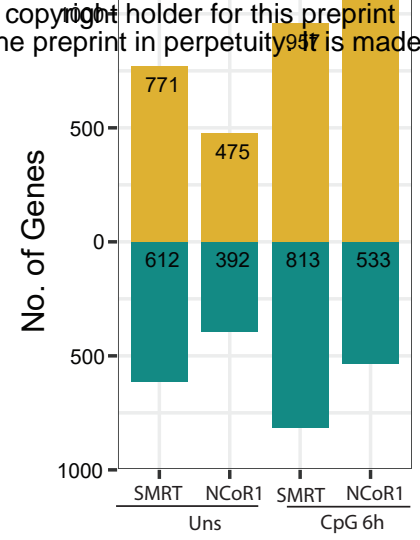
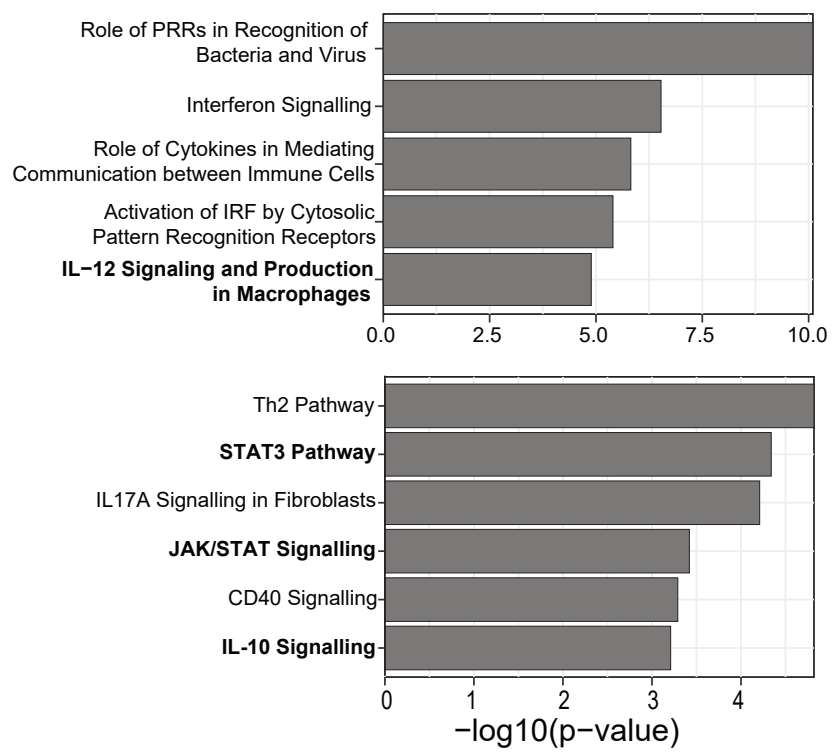

0.0 
Figure 7.

(A) SMRT

NCoR1

(B) Jak/Stat Pathway genes

(C)

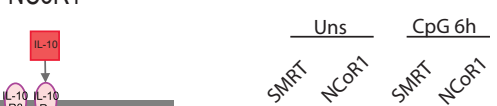

$\begin{array}{lll}\text { UNS } & \text { CpG 2h } \quad \text { CpG 6h }\end{array}$

\begin{tabular}{|c|c|c|c|c|c|c|c|c|}
\hline & \multicolumn{3}{|c|}{ 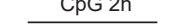 } & \multicolumn{3}{|c|}{ CpG 6} \\
\hline & $Q$ & & & 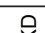 & & & 0 & \\
\hline ò & $\begin{array}{l}\bar{r} \\
\bar{r} \\
\mathrm{O} \\
z\end{array}$ & 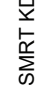 & $\begin{array}{l}\overline{0} \\
\bar{z} \\
0\end{array}$ & $\begin{array}{l}x \\
\bar{r} \\
0 \\
z\end{array}$ & $\begin{array}{l}\frac{v}{2} \\
\sum_{\omega}^{\frac{p}{z}}\end{array}$ & $\begin{array}{l}\overline{0} \\
\overline{0} \\
\overline{0}\end{array}$ & $\begin{array}{l}\bar{g} \\
\overline{0} \\
\bar{z}\end{array}$ & 金 \\
\hline
\end{tabular}

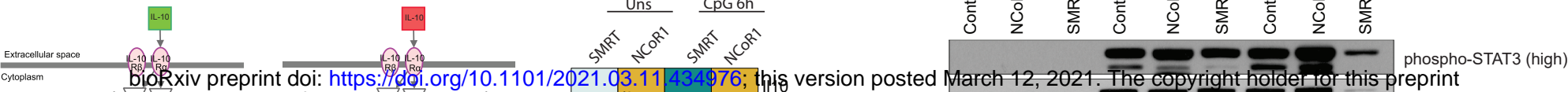

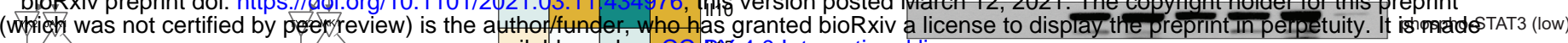

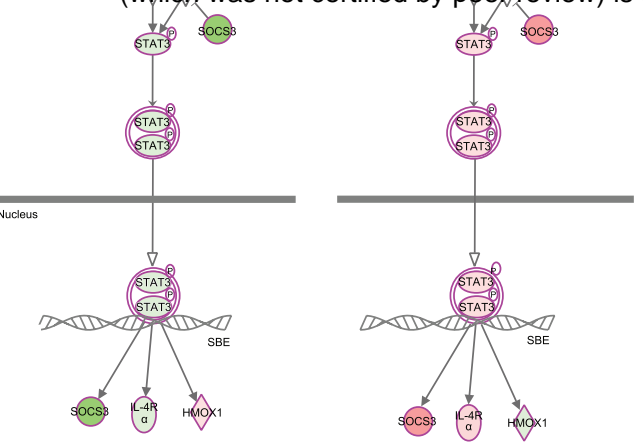

High Expression $\square$ Low Expression

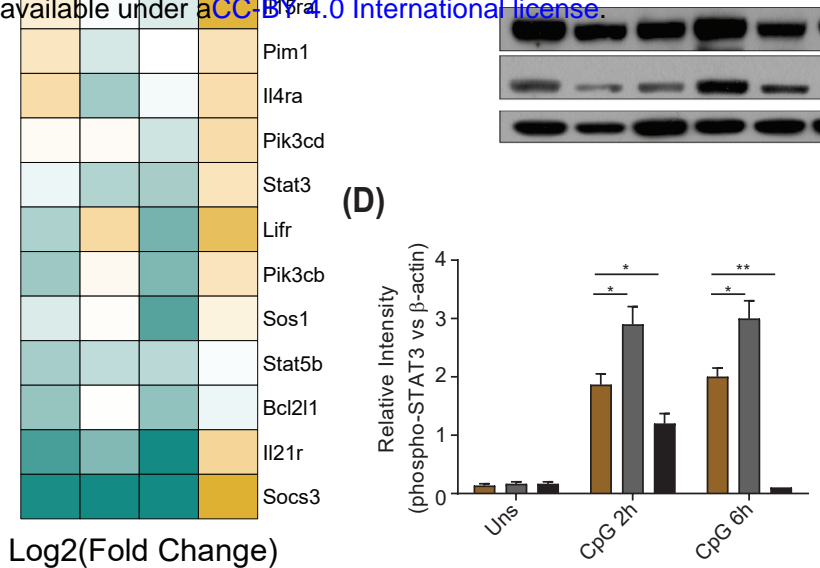

$\square$ Control DCs $\square$ NCoR1 KD DCs
(E)

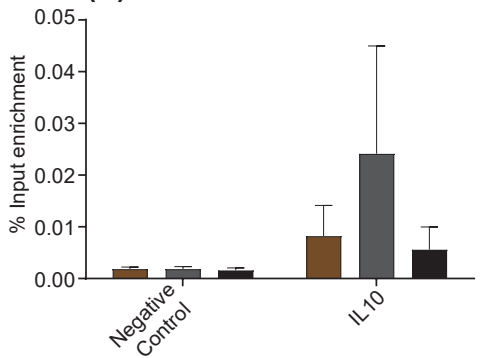

(F)

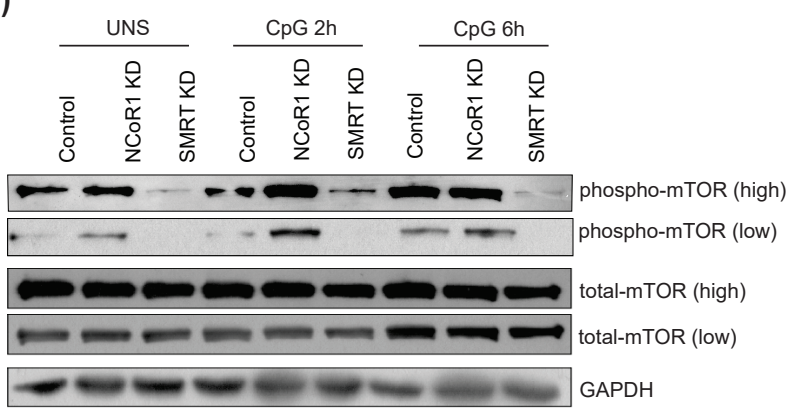

(H) $\square$ Control $\square$ NCoR1 KD $\square$ SMRT KD

(l)

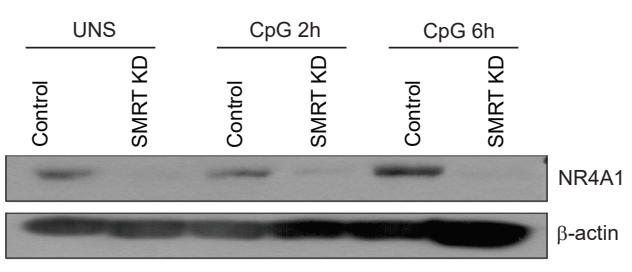

$(\mathrm{J})$

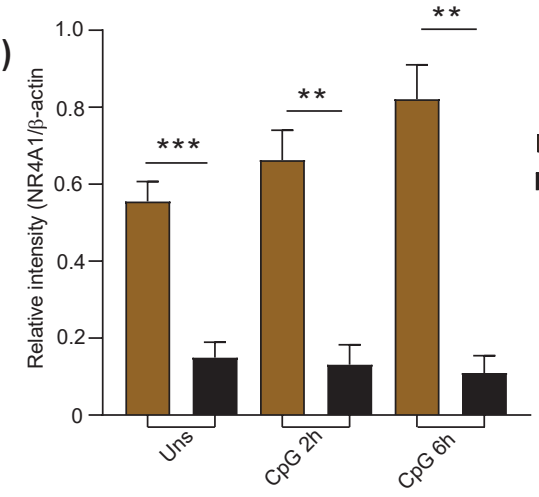

(M) $\quad \mathrm{IL}-10$

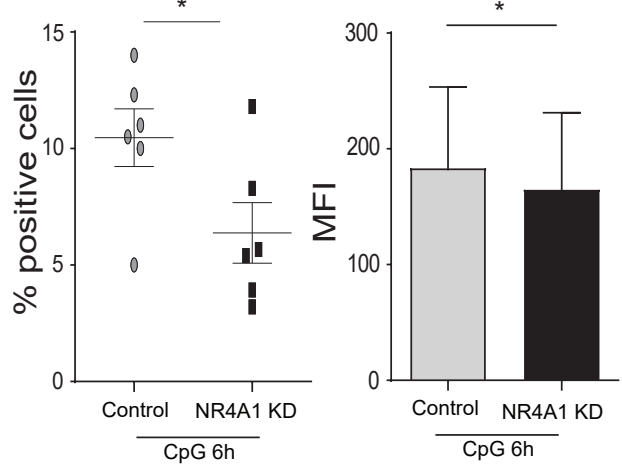

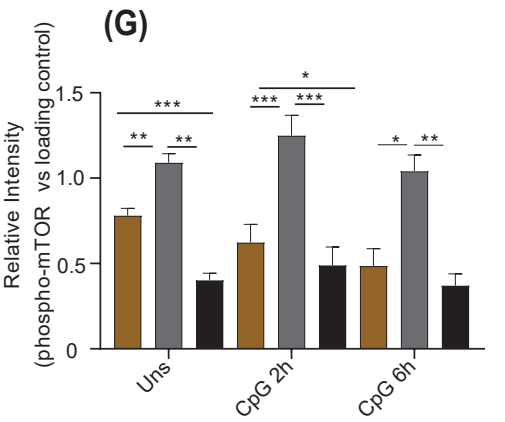

(K)

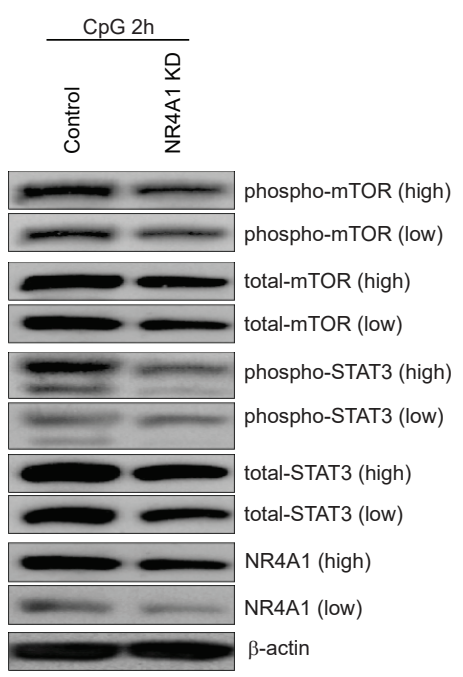

Nr4a1

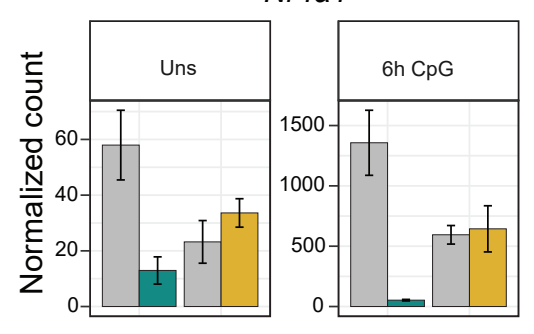

(L) $\square$ Control DCs

- NR4A1 KD DCs
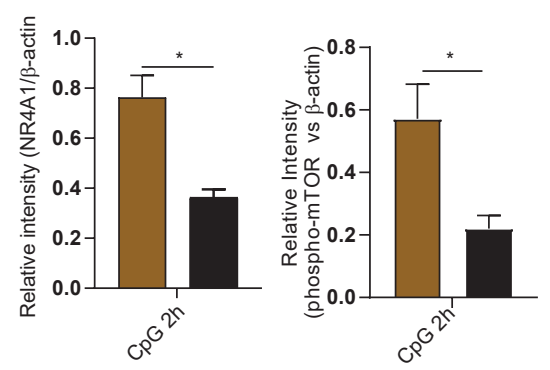

COART

SMRT KD DCs

(N)

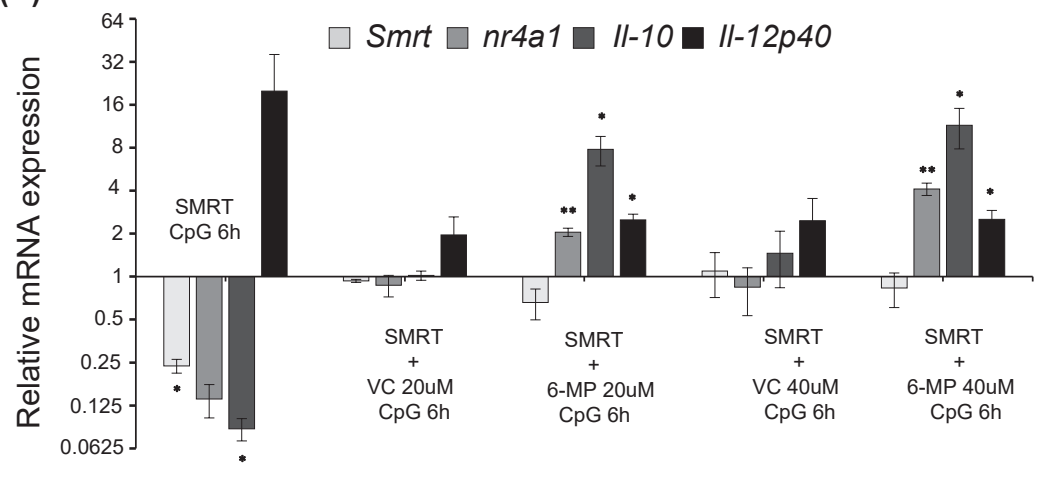




\section{Figure S1}

(A)

Negative Control Control Uns

SMRT KD Uns $\square$ Control CpG $\square$ SMRT KD CpG

Control Uns Control CpG $6 \mathrm{~h}$

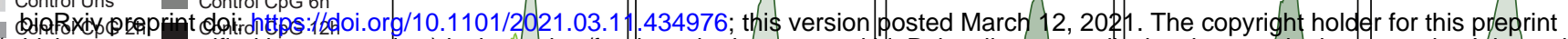

(which was not certified by peer review) is the author/funder, who has granted bioRxiv a license to display the preprint in perpetuity. It is made
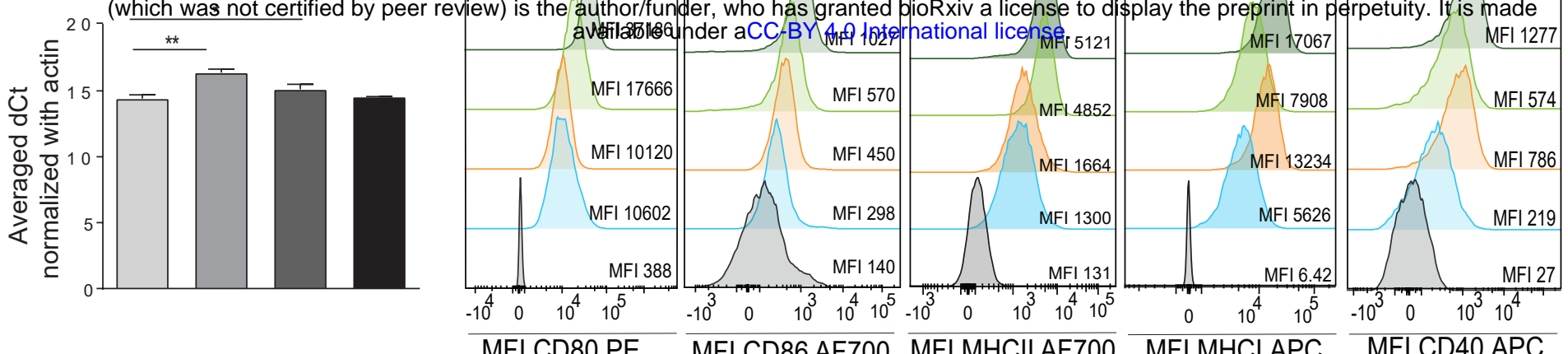

(C)
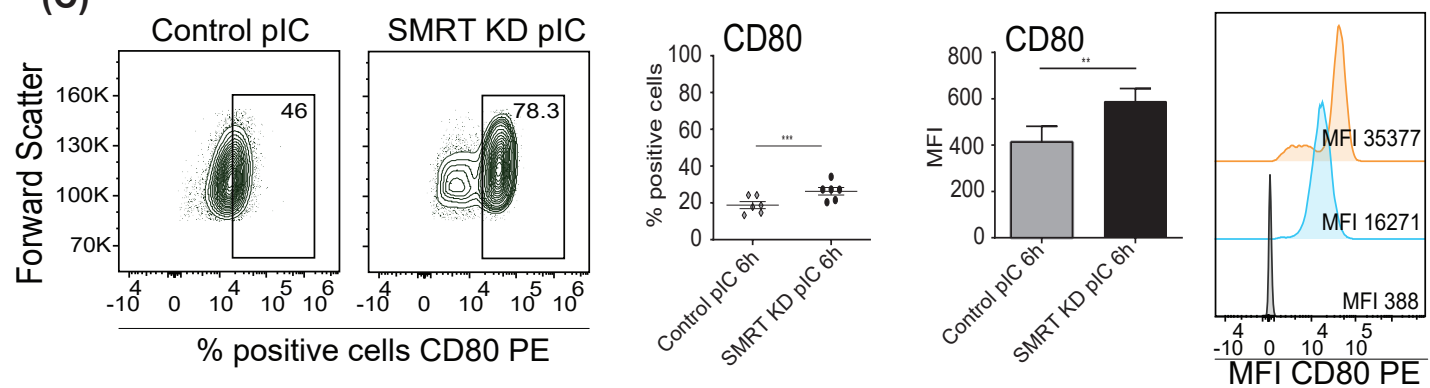

Negative Control

Control pIC

SMRT KD PIC
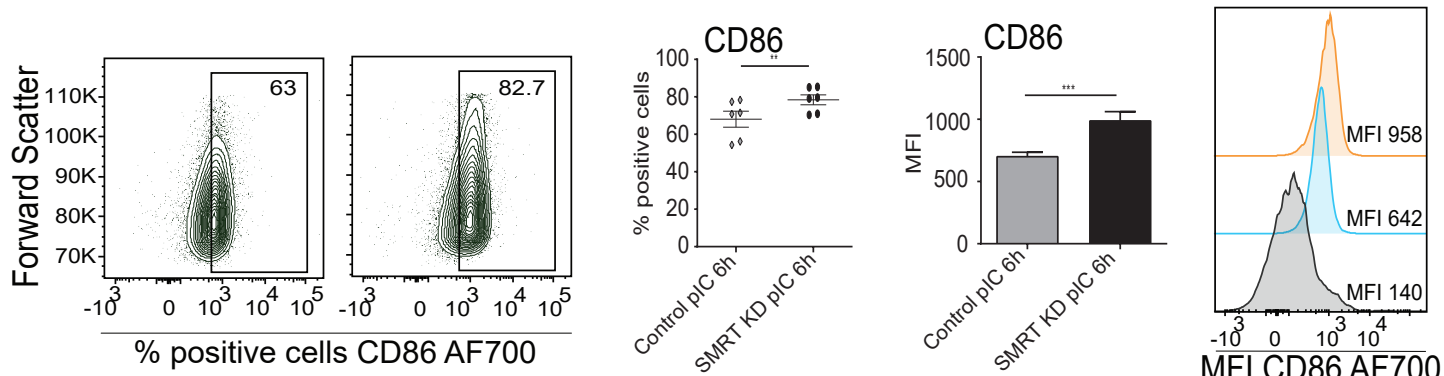

(D)
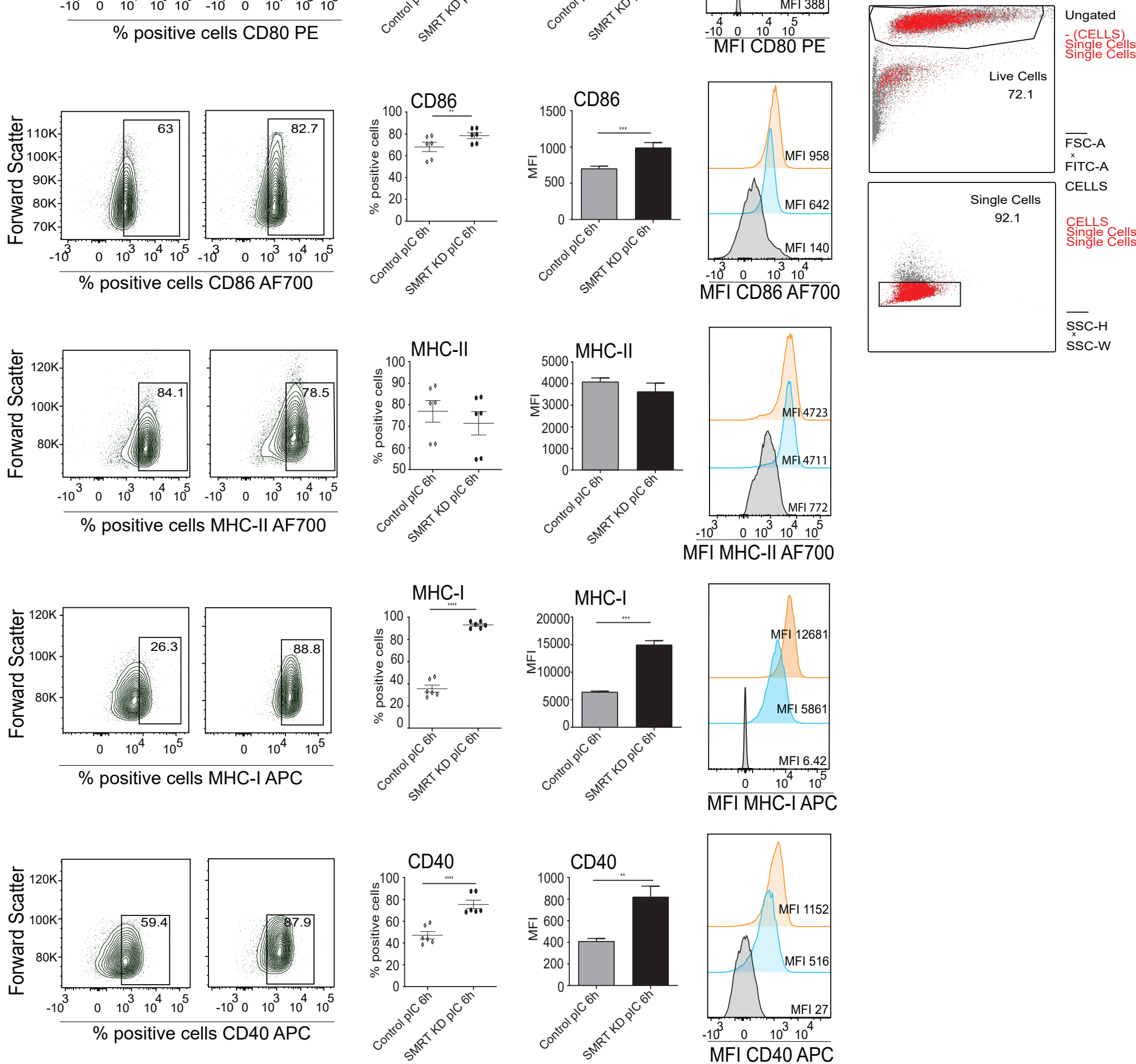
Figure S1. SMRT depleted cDC1 DCs exhibit enhanced co-stimulation and cytokine secretion upon TLR9 and TLR3 stimulation (A) RT-qPCR showing the relative transcript expression of Smrt (averaged dCt normalized

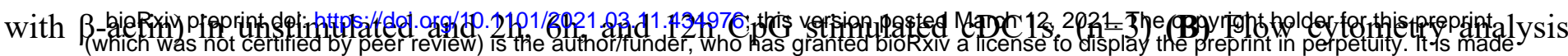
depicting histogram with MFI from flow cy ytometry acnalyysis of con-stimulfatory molecules CD80, CD86, CD40 and activation marker MHC-II, and MHC-I in unstimulated and 6h CpG stimulated control and SMRT KD cDC1s. (C) Flow cytometry analysis of co-stimulatory molecules CD80, CD86, and CD40 as well as activation markers MHC-II and MHC-I in 6h pIC stimulated control and SMRT KD cDC1s. Contour plot, dot plot, bar plot, and histogram showing cell population, percent positive cells and MFI respectively. $(n=6)$ (D) Back gating strategy used throughout the samples.

${ }^{*} \mathrm{p} \leq 0.05, * * \mathrm{p} \leq 0.01$ and $* * * \mathrm{p} \leq 0.001 . \mathrm{p}$-value has been calculated using two tailed paired student's t-test. Data shown in figure is combined from 3 independent experiments [A \& C]. Error bars represent SEM.

\section{Figure S2}

(A)
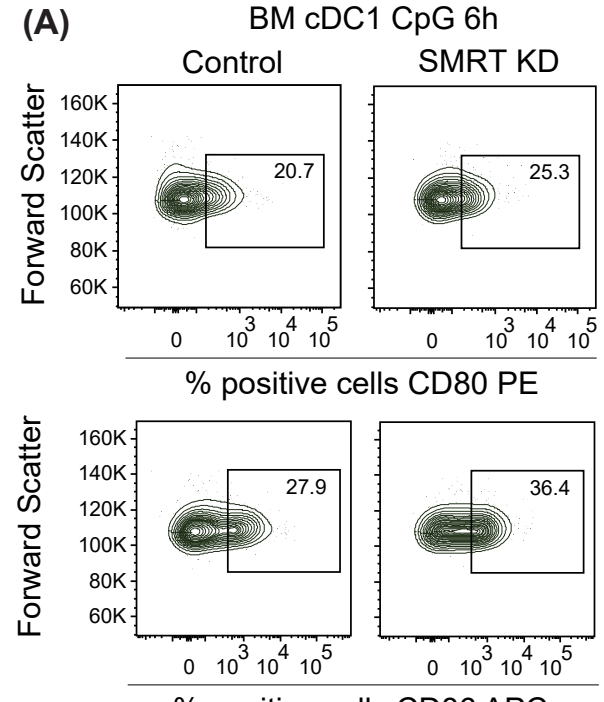

$\%$ positive cells CD86 APC

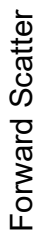

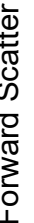

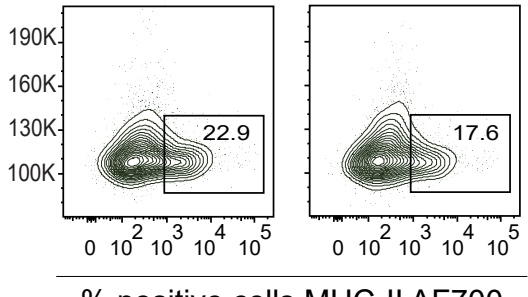

$\%$ positive cells MHC-II AF700
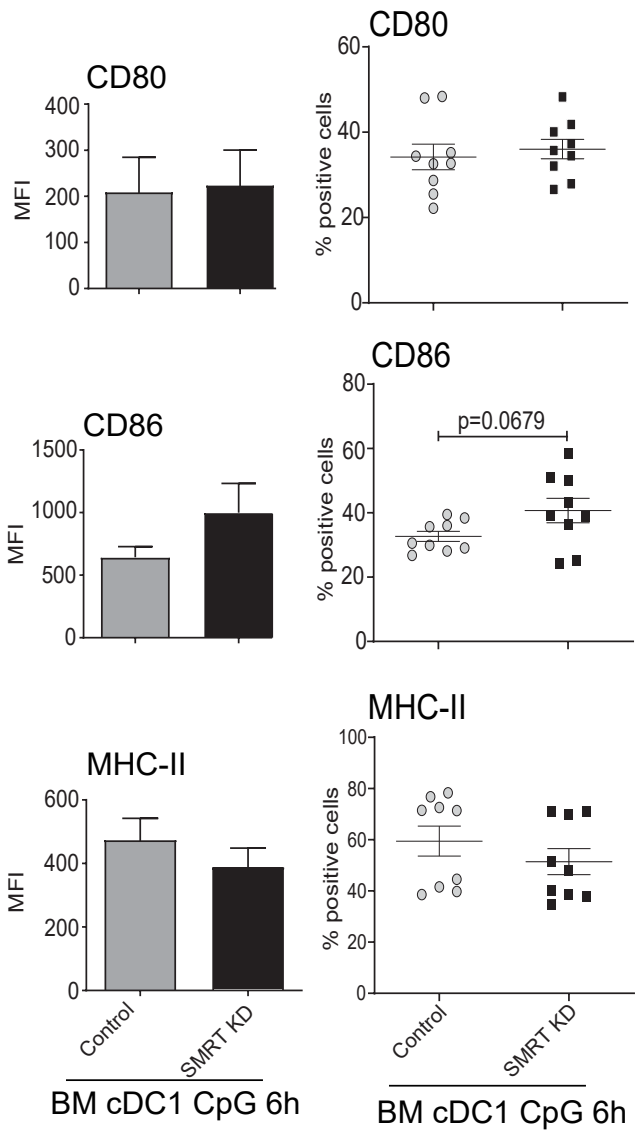

(B)

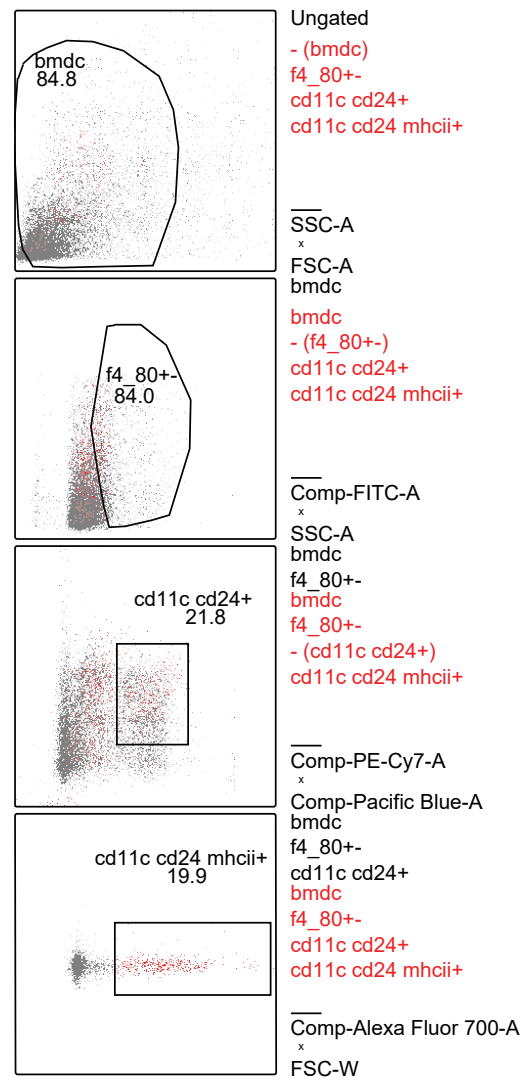

Figure S2. SMRT depleted bone-marrow derived DCs differentiated with FLT3 ligand exhibit enhanced co-stimulation upon CpG stimulation. (A) Flow cytometry analysis of activation and co-stimulatory molecules CD80, CD86, and MHC-II in 6h CpG stimulated control and SMRT KD BMcDC1s. Contour plots, bar plots, and scatter dot plots depicting percent positive cells and MFI. (n=9) (B) Back gating strategy used throughout BMcDC1 samples.

${ }^{*} \mathrm{p} \leq 0.05,{ }^{*} \mathrm{p} \leq 0.01$ and ${ }^{* * *} \mathrm{p} \leq 0.001 . \mathrm{p}$-value has been calculated using two tailed paired student's t-test. Data shown in figure is combined from 4 independent experiments [A]. Error bars represent SEM. 


\section{Figure S3}

(A)

Negative Control $\square$ Control Uns $\quad$ SMRT KD Uns $\square$ Control CpG $\square$ SMRT KD CpG

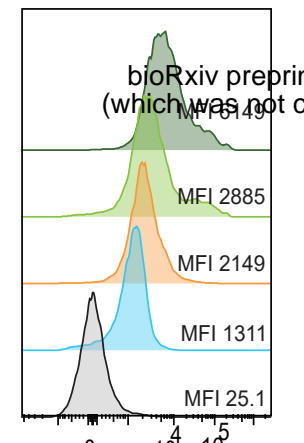

$\begin{array}{lll}0 & 10^{4} & 10^{5}\end{array}$

MFI IL-6 PeCy5
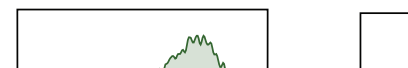

21.03 .11 .43
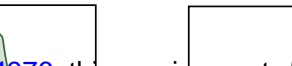

Antorch

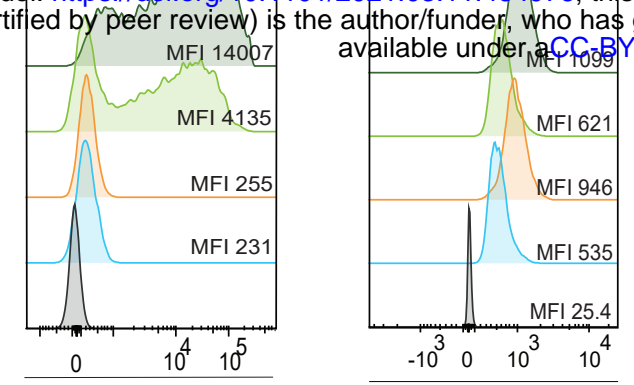

MFI IL-12p40/70 APC MFI IL-23p19 APC

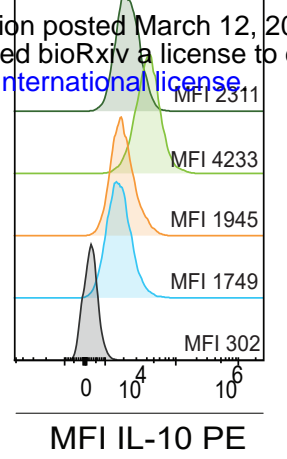

(C)

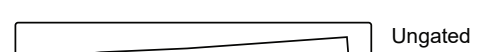

(B)
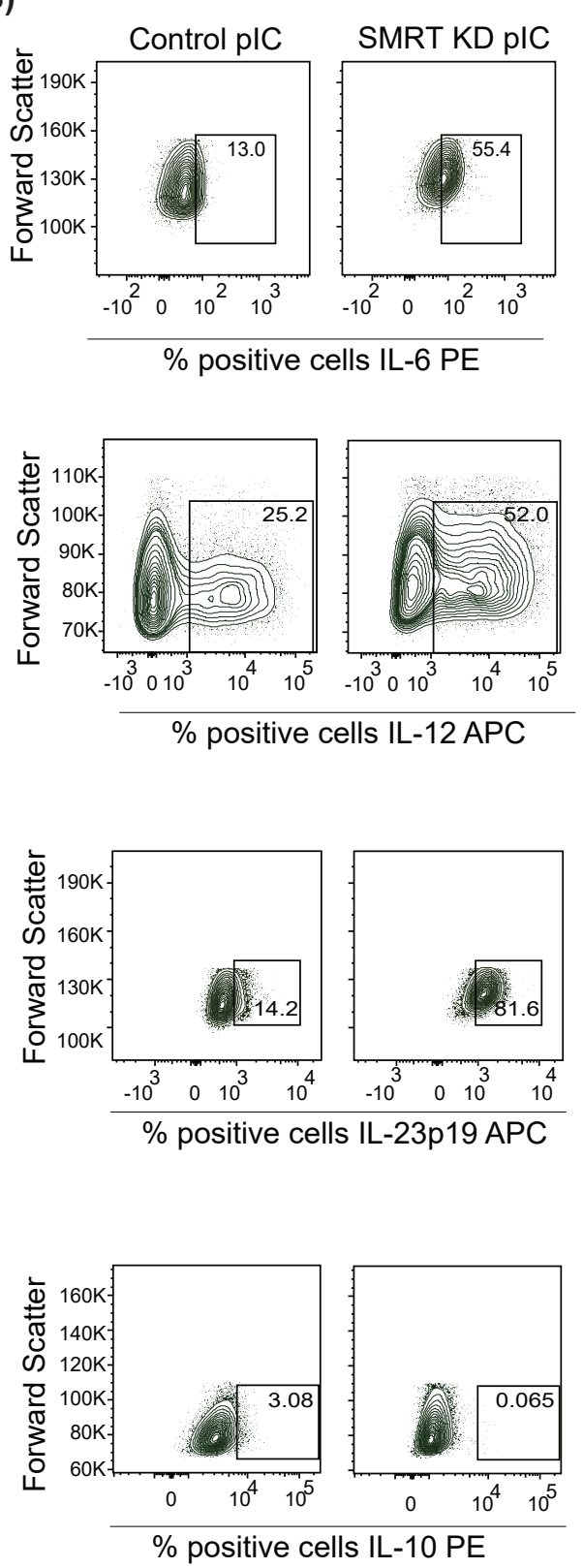
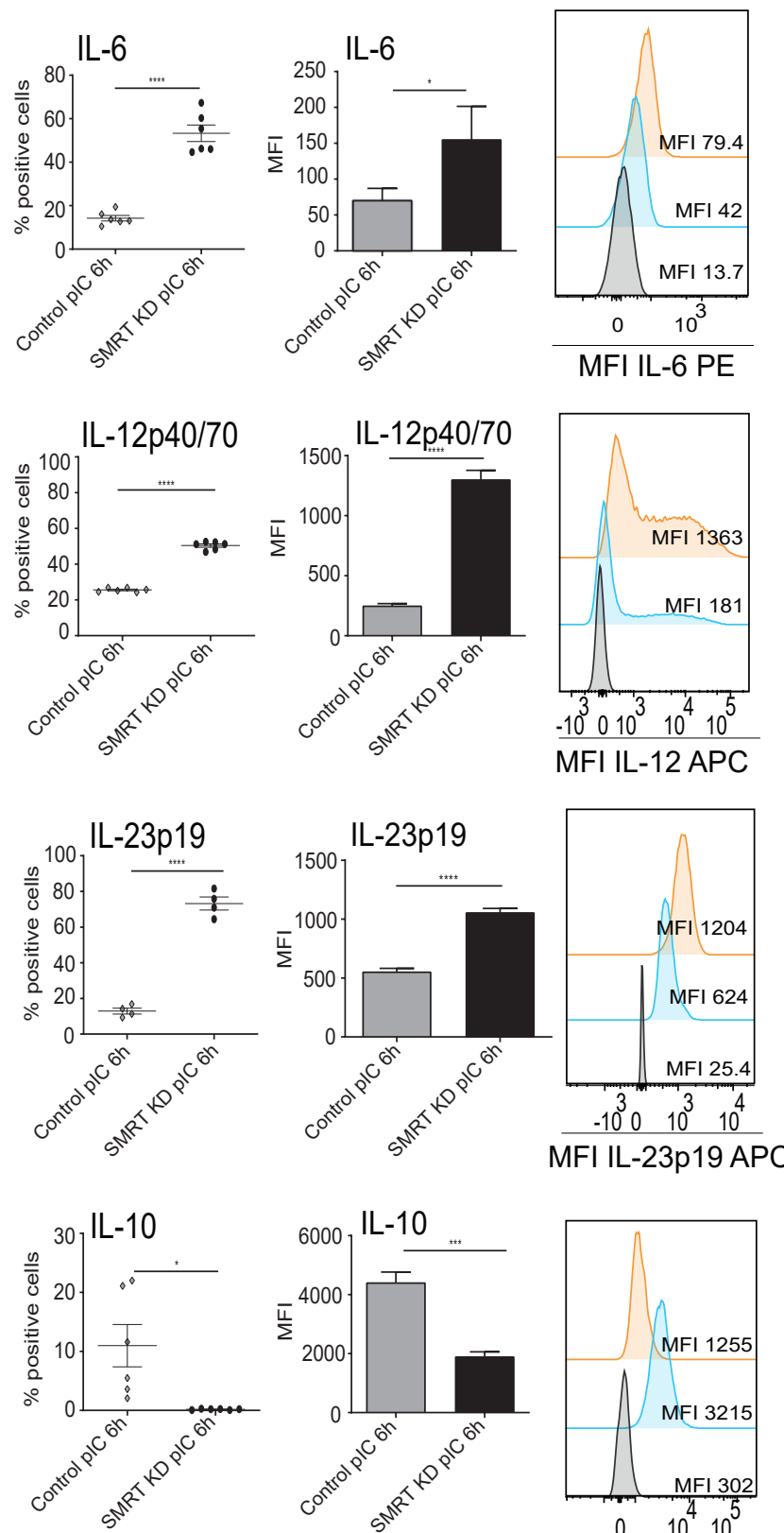
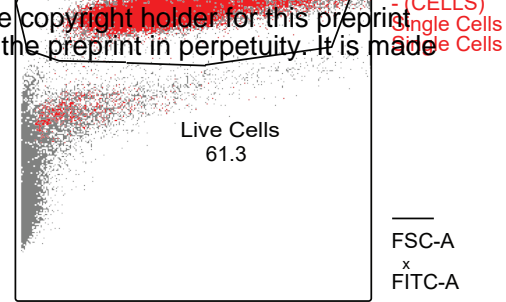

$\overline{\text { FSC-A }}$

FITC-A

CELLS

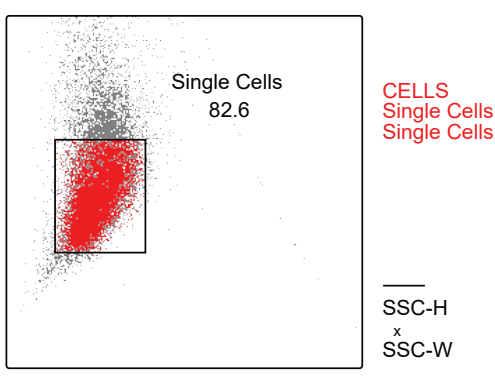

Negative Control

Control plC

SMRT KD pIC

Figure S3. SMRT depleted cDC1 DCs cytokine secretion upon TLR stimulation (A) Flow cytometry analysis showing histograms depicting MFI of pro-inflammatory cytokine IL-6, IL-12p40, IL-23p19, and the anti-inflammatory cytokine IL-10 in unstimulated and 6h CpG stimulated control and SMRT KD cDC1s. (B) Flow cytometry analysis of pro-inflammatory cytokines IL-6, IL-12p40, IL-23p19, and the anti-inflammatory cytokine IL-10 in $6 \mathrm{~h}$ pIC stimulated control and SMRT KD cDC1s. Corresponding contour plot, scatter dot plot, bar plot, and histogram showing cell population, percent positive cells and MFI shifts respectively. $(n=4-6)(C)$ Back gating strategy used for mutuDC analysis.

${ }^{*} \mathrm{p} \leq 0.05, * * \mathrm{p} \leq 0.01$ and ${ }^{* * *} \mathrm{p} \leq 0.001 . \mathrm{p}$-value has been calculated using two tailed paired student's t-test.

Data shown in figure is combined from 2-3 independent experiments [C]. Error bars represent SEM. 
Figure S5

(A)

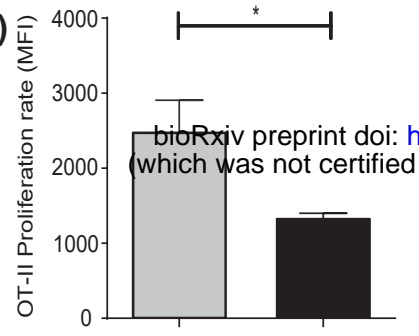

(B)

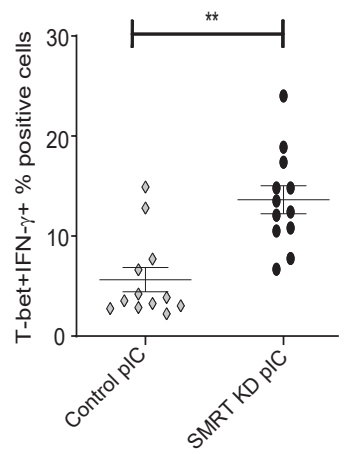

(C)

(F)
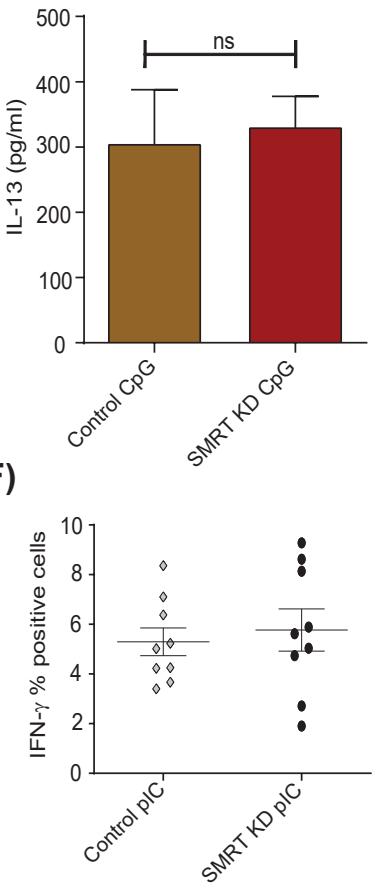

(D)

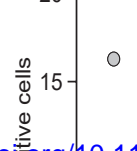

(E)

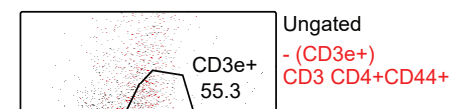

菏rg/10.1101/2021.03.11.434976; this version posted March 12, 2021/The copyright holder for this preprint eview) is the author/funder, who has granted bioRxiv a license to disflay the preprint in perpetuity. It is made
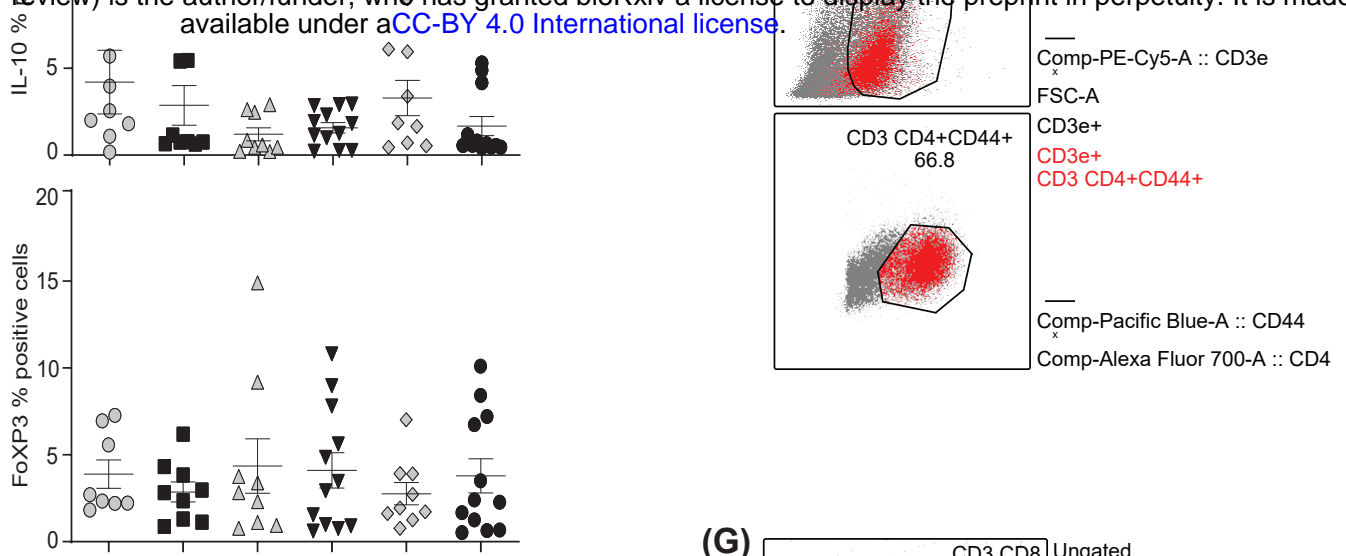

(G)
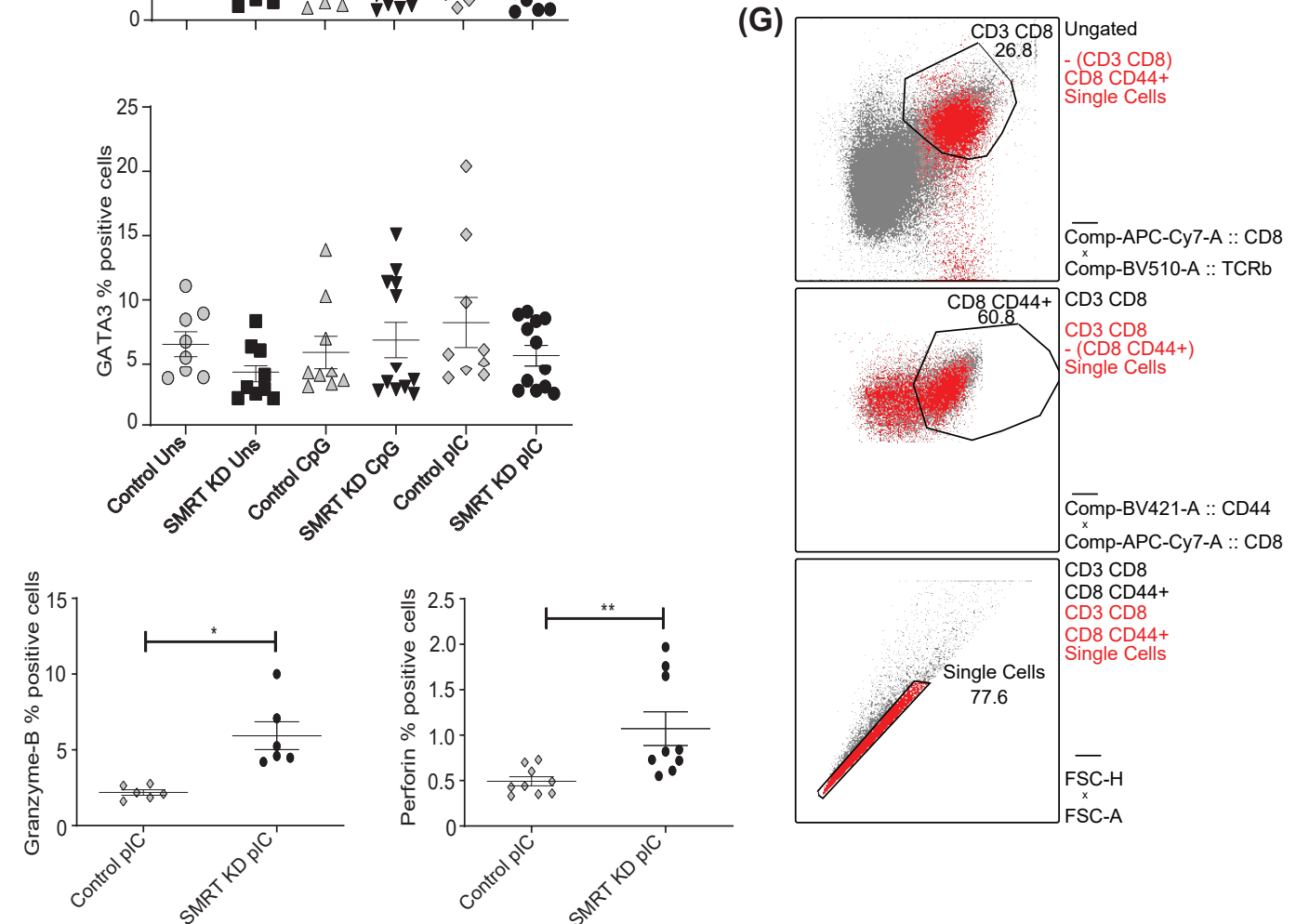

Figure S5. SMRT KD cDC1s increased Th1 and Th17 polarization ex vivo in CD4 ${ }^{+}$T-lymphocytes and $^{-l y}$ enhanced cytotoxic T-cell activity in CD8 $^{+}$lymphpcytes (A) MFI shifts of proliferation dye eF670 depicting the difference in proliferation rate of OT-II Th-cells co-cultured with control and SMRT KD cDC1s treated with OVA 323-339 peptide overnight followed by pIC stimulation. $(n=5)(B)$ Dot plots representing co-cultured OT-II Th-cells showing signature transcription factor and cytokine for Th1, T-bet and IFN- $\gamma$ response in pIC stimulated condition. $(\mathrm{n}=12)(\mathrm{C})$ Bioplex cytokine assay depicting quantification of Il-13 cytokine secreted in the culture supernatant of OT-II Th-cells which were co-cultured with control and SMRT KD DCs pulsed with OT-II peptide and challenged with CpG. (n=5) (D) Dot plots representing co-cultured OT-II Th-cells showing IL-10, FoxP3, and GATA3 expressing T-cells co-cultured with control and SMRT KD DCs in unstimulated, CpG stimulated, and pIC stimulated condition. (n=8-12). (E) Back gating strategy used for flow cytometry analysis of co-cultured OT-II T-cells. (F) Dot plots representing co-cultured OT-I T-cells showing cytokines for cytotoxic T-cells perforin, granzyme-B, and IFN- $\gamma$ in pIC stimulated condition. ( $\mathrm{n}=6-9)$ (G) Back gating strategy used for flow cytometry analysis of co-cultured OT-I T-cells.

${ }^{*} \mathrm{p} \leq 0.05, * * \mathrm{p} \leq 0.01$ and $* * * \mathrm{p} \leq 0.001 . \mathrm{p}$-value has been calculated using two tailed paired student's t-test. Data shown in figure is combined from 2 independent experiments [A], from 4 independent replicates [B], from 3 independent replicates [C], from 2 independent replicates [D], and 2-4 independent replicates [F]. Error bars represent SEM. 
Figure S6

(A) $\quad$ PBS Control SMRT KD
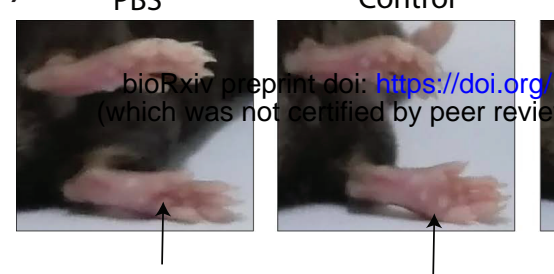

10.1101/2021.03.11.434976; this version posted March 12, 2021. The copyright holder for this preprint is the author/funder, who has granted bioRxiv a license to display the preprint in perpetuity. It is made available under aCC-BY 4.0 International license.

(B)

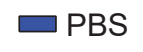

Control DC

- SMRT KD DC
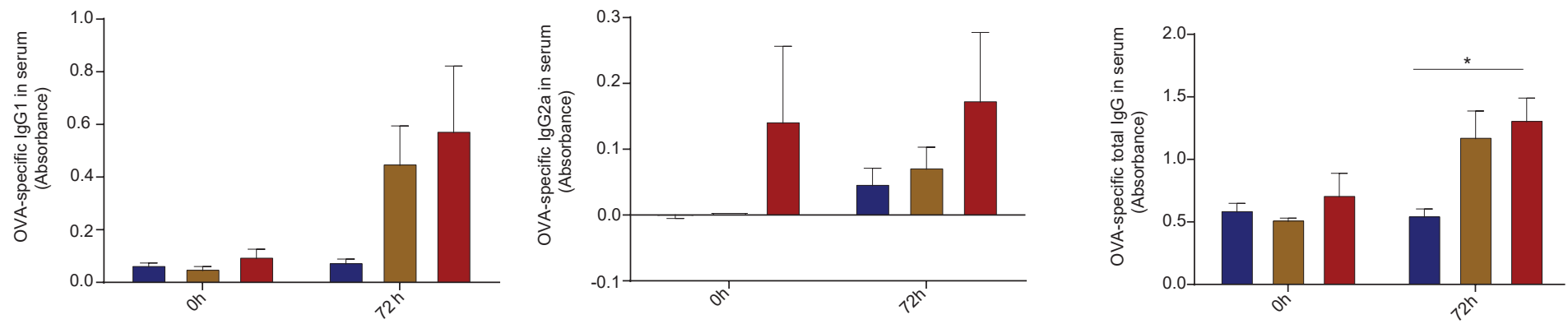

(C)

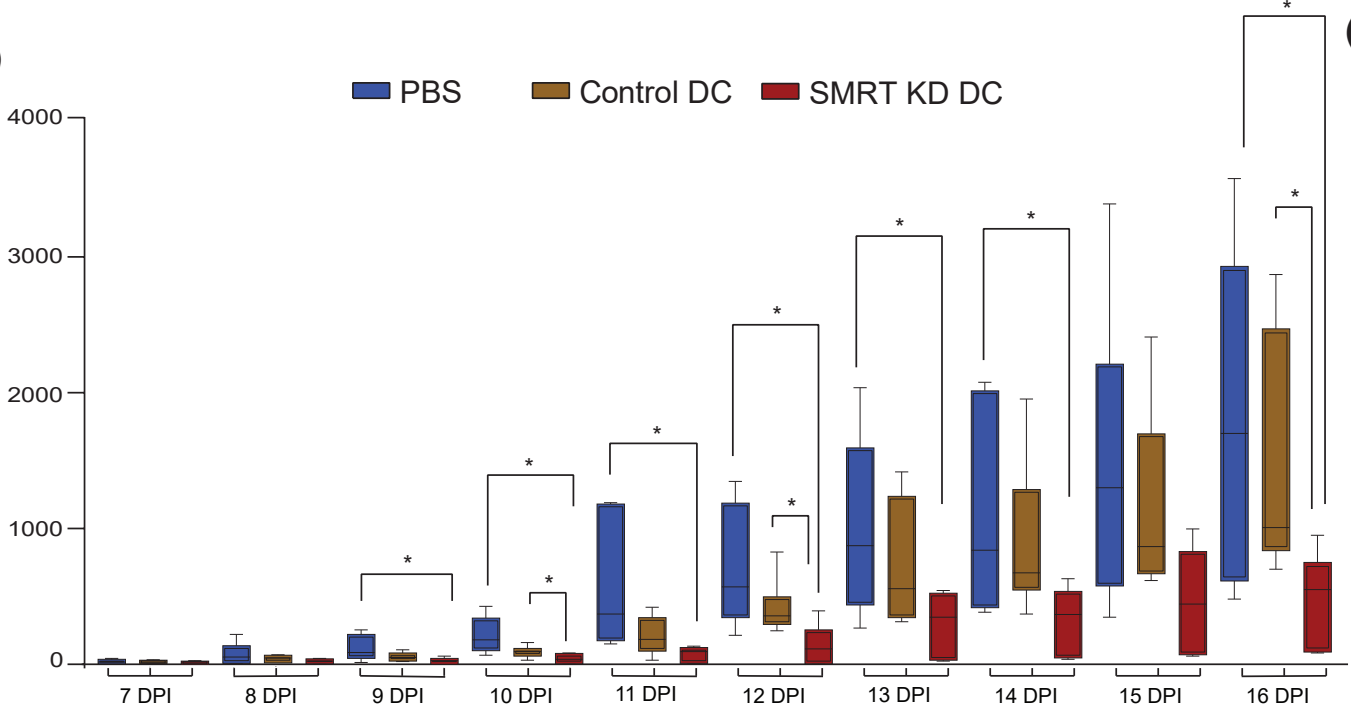

(D)
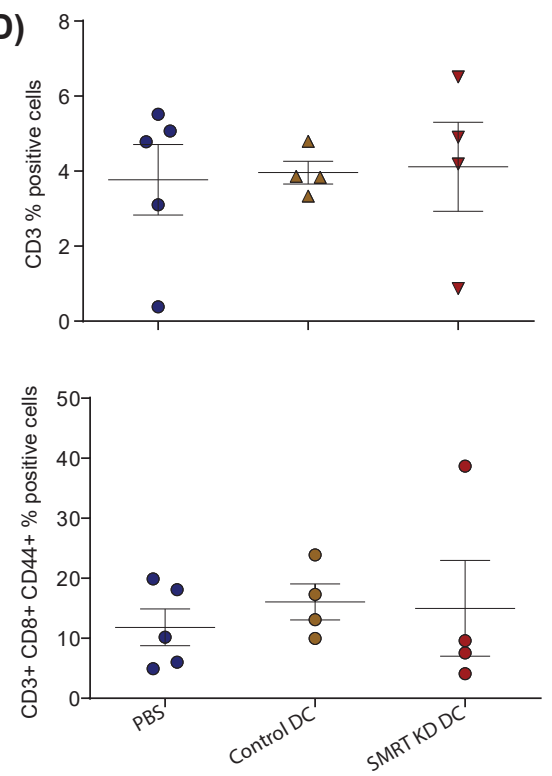

(E)
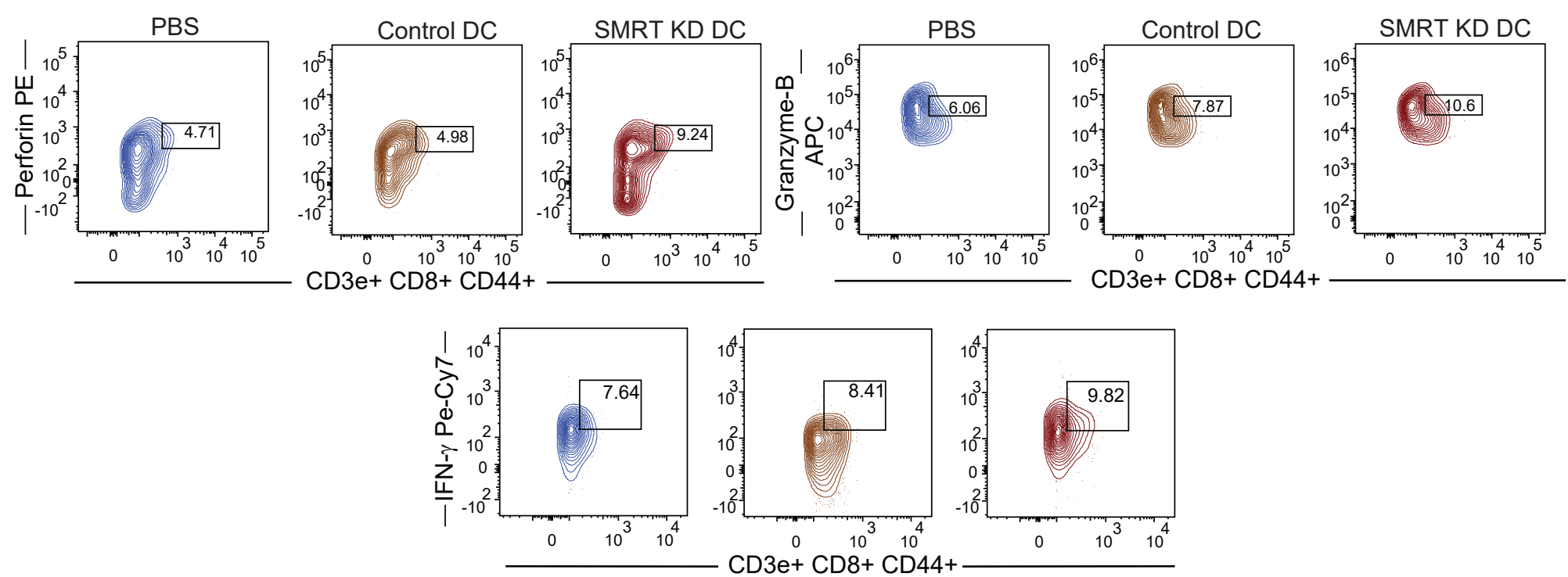

Figure S6. Induction of DTH and B16F10 induced melanoma model in C57BL/6 (A) Image showing footpad swelling from PBS, ova pulsed control DCs, and SMRT KD DCs treated mice $72 \mathrm{~h}$ post ova rechallenge. (B) ELISA of IgG1, IgG2a, total IgG in 0 and $72 \mathrm{~h}$ after OVA immunization in in mice injected with PBS, ova pulsed control DCs, and SMRT KD DC. $(n=4-6)$ 
(C) Box plot showing tumor volume that was taken every day starting from 7th day after B16F10 injection till 16 days post tumor rechallenge in PBS, control and SMRT KD DCs injected mice. (D) Dot plots depicting percentage of CD ${ }^{+}$

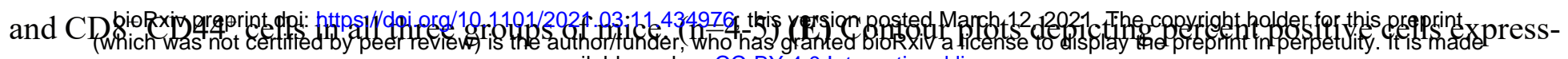
ing perforin, granzyme-B, and IFN- $\gamma$ in thrailable under aCc-BY.4.0 International license.

${ }^{*} \mathrm{p} \leq 0.05, * * \mathrm{p} \leq 0.01$ and $* * * \mathrm{p} \leq 0.001$. $\mathrm{p}$-value has been calculated using two tailed unpaired student's t-test. Data shown in figure is combined from 2 independent experiments [A-C] and 1 replicates [D]. Error bars represent SEM. 


\section{(Figure S7)}
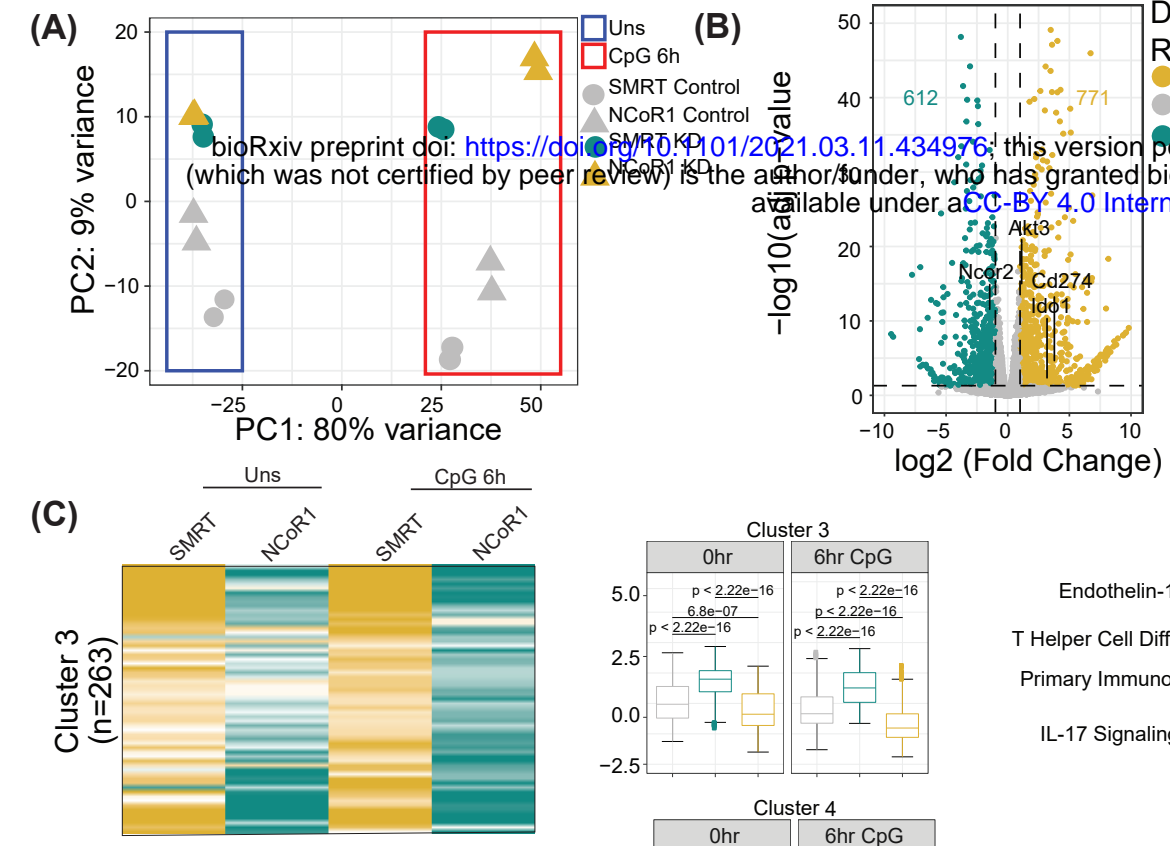

Differential
Regulation
Up
No Change

(E)

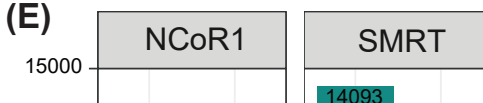

No Change

(Fold Change)

(D)

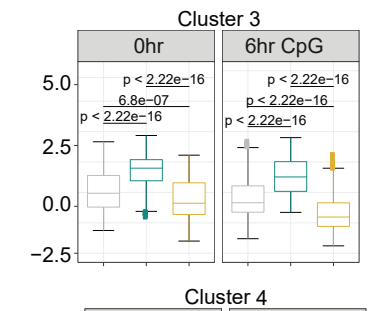

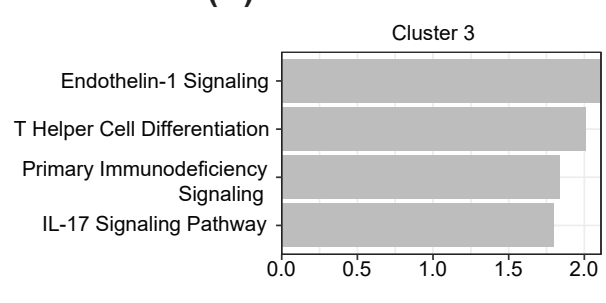

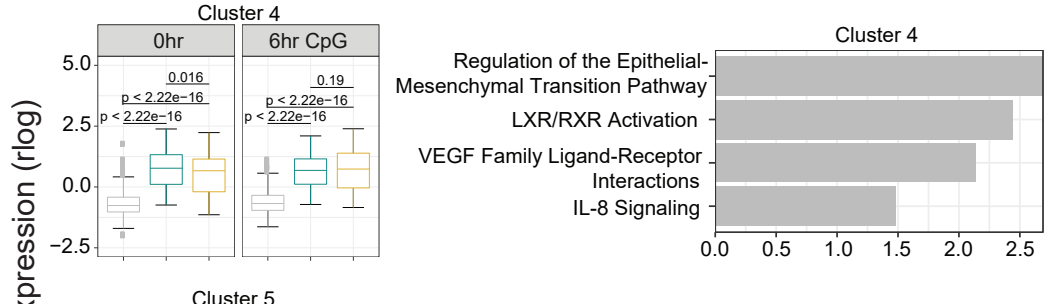

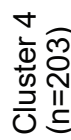
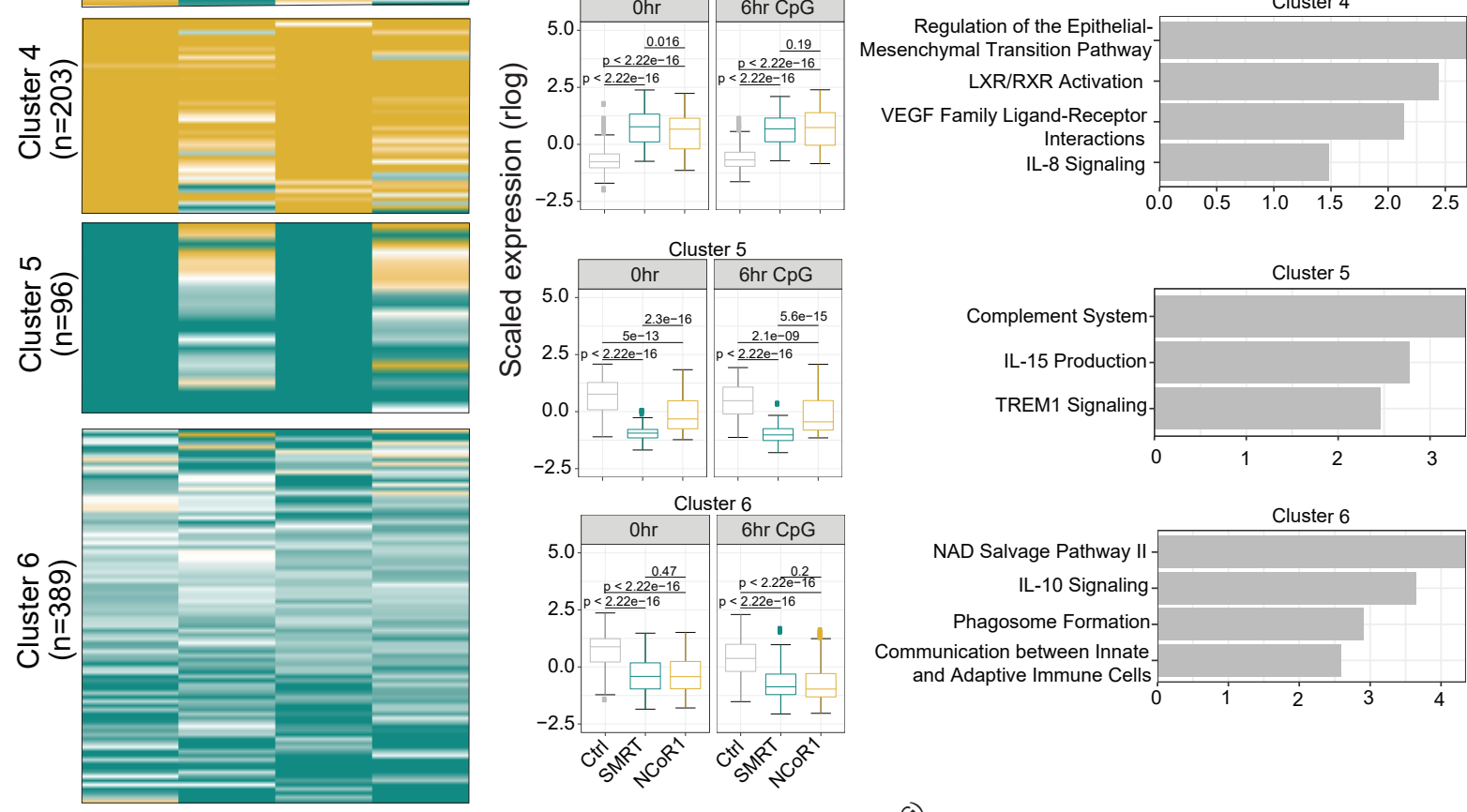

(F)
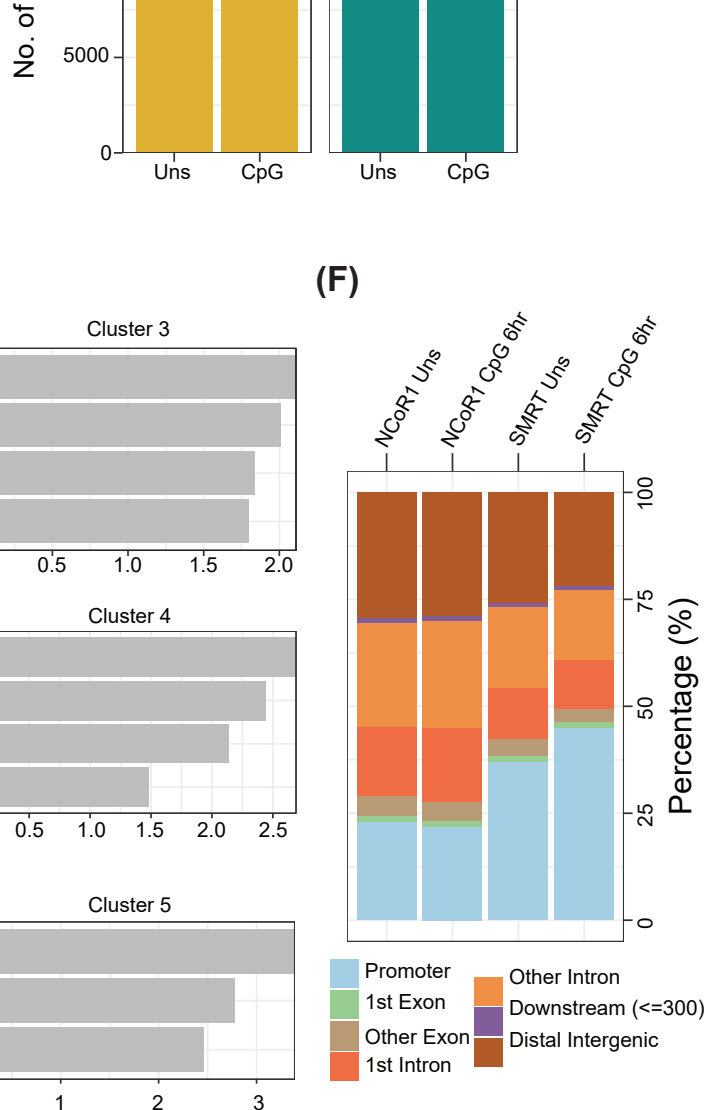

Cluster 6
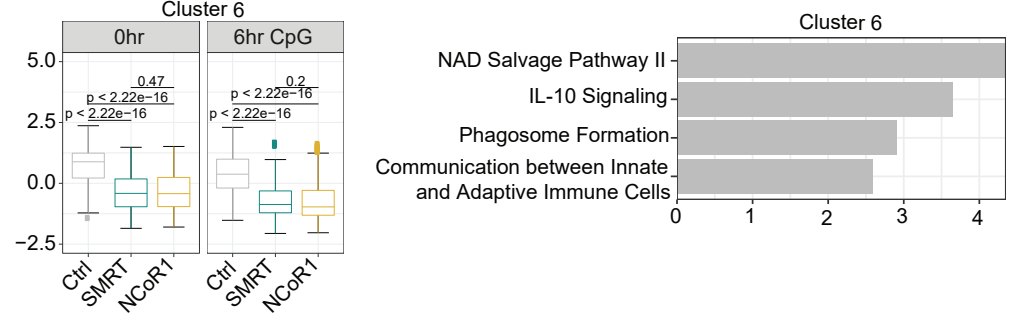

(G)

(H)

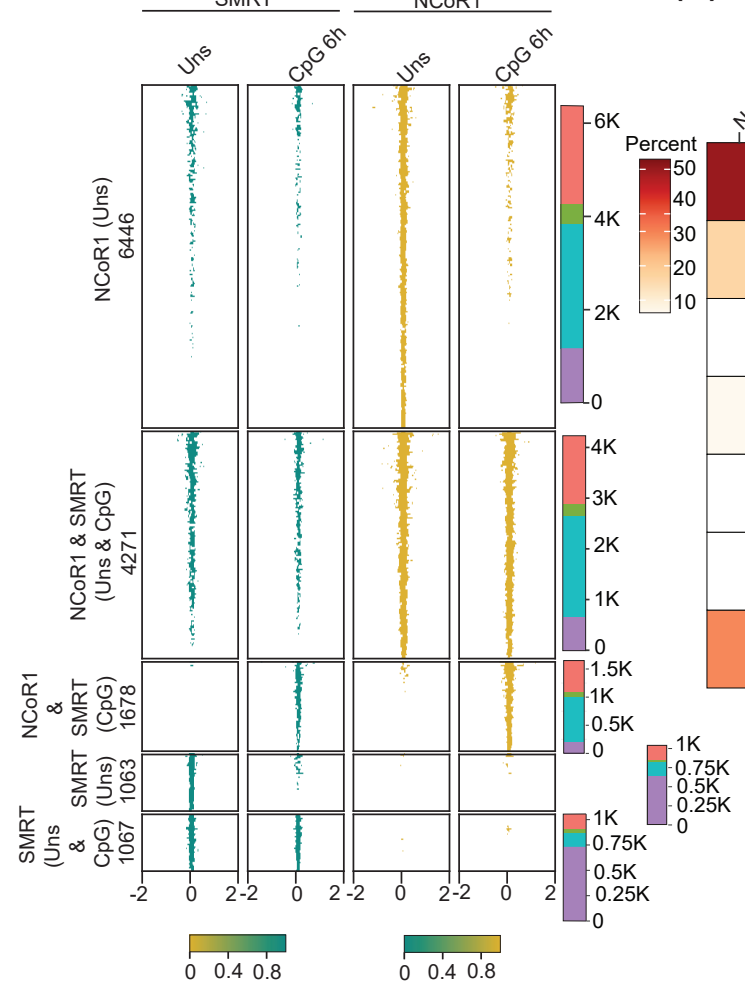

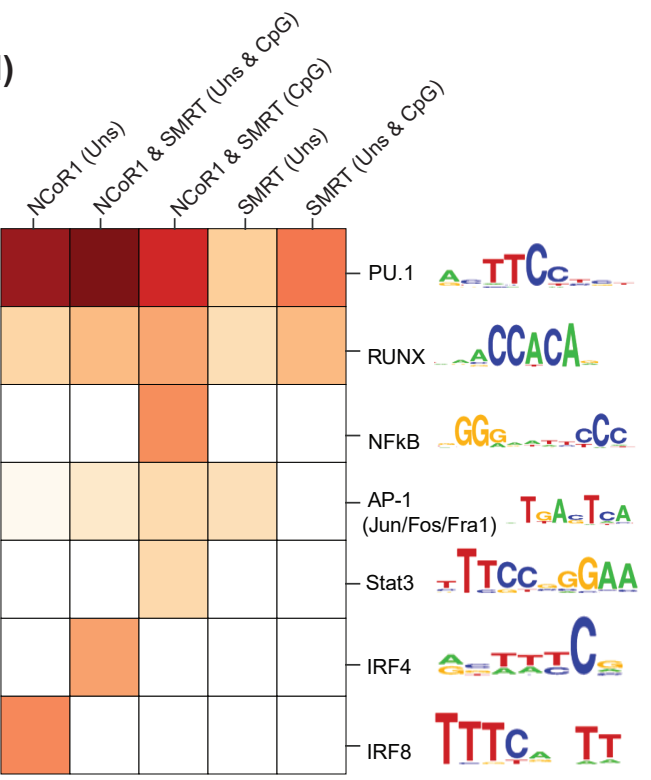

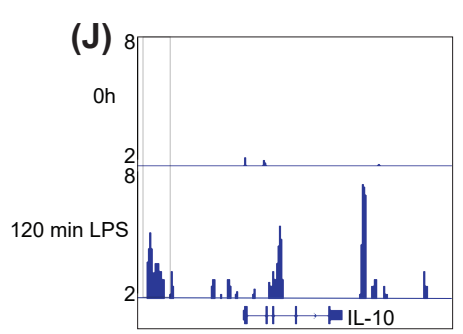

(I)

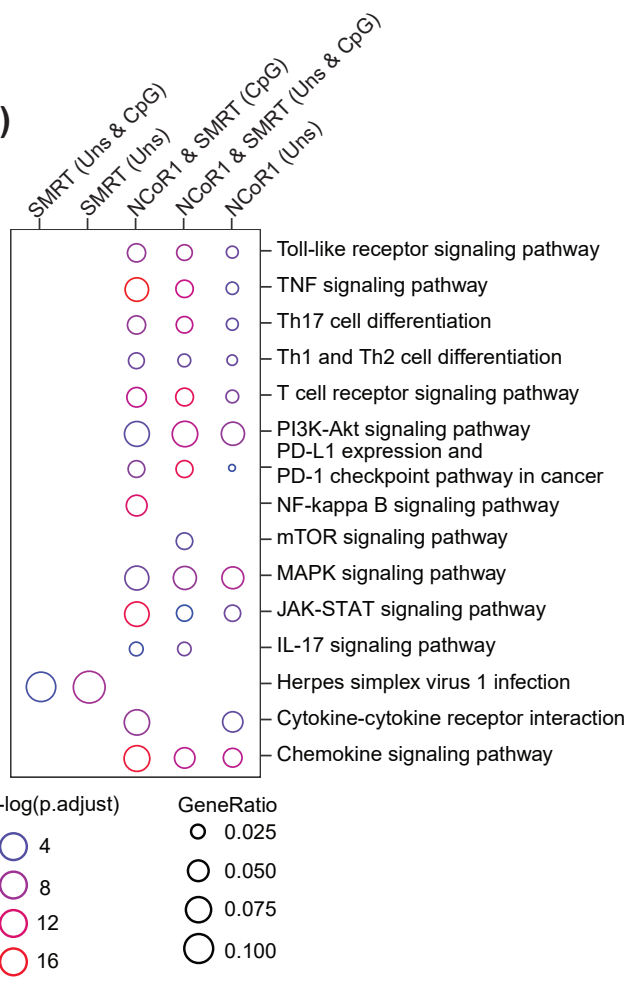


Figure S7. Integrative genomics analysis identified the differential role of SMRT and NCoR1 in regulation of immune response in cDC1. (A) PCA plot showing the percent of the variation in principal component 1 and

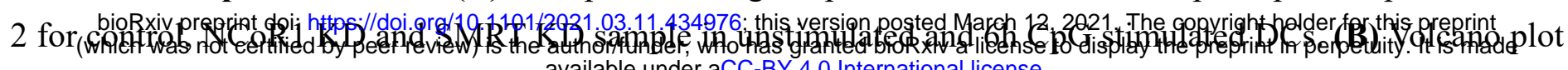
showing the differentially expressed genes (DEGS) in SMRT KD cDC1 DCs as compared to control cells in unstimulated condition. 771 and 612 genes were upregulated and downregulated respectively upon SMRT depletion. (C) Heatmap showing clusters 3-6 of K-means clustering (shown in Fig 6F) of log2 fold change of DEGs upon SMRT and NCoR1 KD compared to control cells in unstimulated and 6h CpG stimulated DCs. The box plot shows scaled normalized expression values (rlog) from DESeq2 for the respective cluster. (D) The bar plot showing enriched pathway terms from Ingenuity pathway analysis for respective clusters. (E) Bar plot showing the total number of identified peaks (binding sites) of NCoR1 and SMRT in unstimulated and 6h CpG stimulated DCs. (F) Percentage stacked bar plot showing the global distribution of NCoR1 and SMRT identified peaks based on distance relative to TSS in unstimulated and $6 \mathrm{~h} \mathrm{CpG} \mathrm{stimulation.} \mathrm{(G)} \mathrm{Tornado} \mathrm{plot} \mathrm{showing} \mathrm{ChIP-seq} \mathrm{signal}$ ( $\pm 2 \mathrm{~kb}$ to peak center) of differential NCoR1 and SMRT binding sites in unstimulated and $6 \mathrm{~h}$ CpG stimulation. Bar plot showing the distribution of differential genomic regions based on distance relative to TSS. (H)

Heatmap showing percent of target binding sites with transcription factor motifs that were significantly enriched (P-value $<1 \mathrm{e}-10$ ) in differential NCoR1 and SMRT genomic regions. (I) Dot plot showing the enriched KEGG terms for genes associated with differential NCoR1 and SMRT binding clusters (genomic regions). (J) IGV snapshot showing ChIP-seq enrichment of STAT3 on Il-10 gene in BMDCs at 0h and 120 min post LPS stimulation. 